\title{
MECHANICS OF LIGHT WEIGHT PROPPANTS: A DISCRETE APPROACH
}

\author{
A Dissertation \\ by \\ MANDAR CHAITANYA KULKARNI \\ Submitted to the Office of Graduate Studies of \\ Texas A\&M University \\ in partial fulfillment of the requirements for the degree of \\ DOCTOR OF PHILOSOPHY
}

May 2012

Major Subject: Mechanical Engineering 


\title{
MECHANICS OF LIGHT WEIGHT PROPPANTS: A DISCRETE APPROACH
}

\author{
A Dissertation \\ by \\ MANDAR CHAITANYA KULKARNI
}

\author{
Submitted to the Office of Graduate Studies of \\ Texas A\&M University \\ in partial fulfillment of the requirements for the degree of \\ DOCTOR OF PHILOSOPHY
}

\begin{abstract}
Approved by:
Chair of Committee, Ozden Ochoa

Committee Members, Anastasia Muliana

J.N. Reddy

Theofanis Strouboulis

Zenon Medina-Cetina

Head of Department, Jerald A. Caton
\end{abstract}

May 2012

Major Subject: Mechanical Engineering 


\begin{abstract}
Mechanics of Light Weight Proppants: A Discrete Approach. (May 2012)

Mandar Chaitanya Kulkarni, B.E., Sardar Patel University, India;

M.S., Texas A\&M University

Chair of Advisory Committee: Dr. Ozden Ochoa
\end{abstract}

Proppants are a specific application of granular materials used in oil/gas well stimulation. Employment of hard and soft particle mixtures is one of the many approaches availed by the industry to improve fracture resistance and the stability of the granular pack in the hydraulic fracture. Current industrial practices of proppant characterization involve long term and expensive conductivity tests. However, the mechanics governing the proppant pack response, in particular the effects due to material, shape and size of particles on the pack porosity, stiffness and particle fragmentation are not understood clearly.

The present research embodies analytical and experimental approach to model hard (ceramic) and soft (walnut shell and/or pure aluminum) proppant mixtures by taking into account polydispersity in size, shape and material type of individual particles. The hydraulic fracture condition is represented through confined compression and flowback loads. The particle interactions clearly illustrate changes in pore space as a function of pressure, mixture composition and friction. Single particle compression tests on individual particles are carried out to obtain mechanical properties which are incorporated into the finite element models and are further correlated with the compression/crush response of the mixture. The proppant pack stiffness and particle fragmentation depends strongly on the mixture composition as illustrated in the models and experiments. The flowback models demonstrated that the formation of a stable arch is essential to pack stability. Additional variables that enhance flowback resistance are identified as; addition of softer particles to a pack, softer rock surfaces and higher interparticle friction. The computational studies also led to the discovery of better, and more 
efficient pack compositions such as - short and thin pure Al needles/ceramic and the pistachio shells/ceramic mixtures. These analytical results have generated great interest and are engaged in the design of experiments to formulate future proppant pack mixtures at Baker Hughes Pressure Pumping, Tomball, TX. 


\section{ACKNOWLEDGMENTS}

First of all I would like to thank Dr. Ozden Ochoa for giving me an opportunity to work on this project. It has been a privilege to work under her guidance. She has always been there to guide me with the research and has been very patient in her explanations and discussions. Working with her has been a great learning experience and she has continuously inspired and motivated me to work towards my academic goals.

I would also like to thank Dr. Theofanis Strouboulis, Dr. J. N. Reddy, Dr. Zenon Medina-Cetina and Dr.Anastasia Muliana for serving on my committee and providing me with valuable guidance during the course of my work.

I am also thankful to my friend Udaya Bhanu Sunku from the Department of Aerospace Engineering, Texas A\&M University for his invaluable help and guidance in developing the Matlab code to generate an initial random particle distribution.

I would also take this opportunity to thank my past and present lab mates, Melanie, Douglas, Min, Nori and Hieu for being a constant source of inspiration and help. The discussions I had with you all helped advance my research further.

I have to mention my dear friends, Nikhil and Sneha, who have always been there to help and encourage. And of course I have to mention my dear wife Priya who has been so patient and supportive this past year. You have been a solid support and a great source of motivation.

I dedicate this work to my parents whose guidance and blessings have ensured that I reach this position. Their encouragement and support right through my academic career has enabled me to grow and progress. I bow to them with all respect and dedicate this work to them.

I also gratefully appreciate the research guidance and experimental facilities provided by Baker Hughes Pressure Pumping, Tomball, TX. 


\section{TABLE OF CONTENTS}

Page

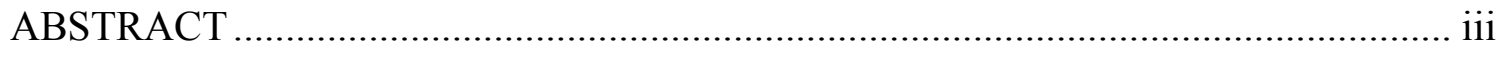

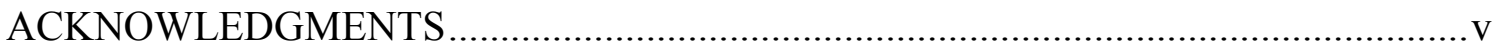

TABLE OF CONTENTS ........................................................................................... vi

LIST OF FIGURES ........................................................................................ viii

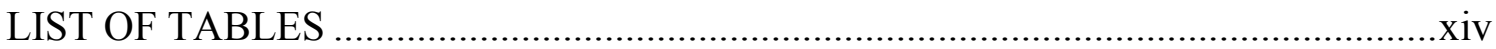

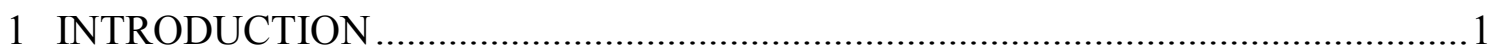

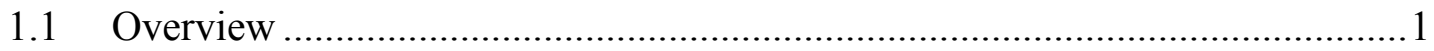

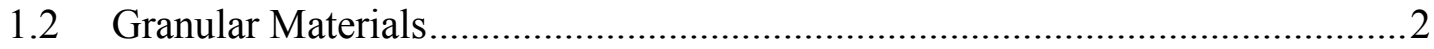

1.3 Computational Techniques to Model Granular Materials ................................5

1.3.1 Continuum Approach ....................................................................... 5

1.3.2 Discrete Approach .................................................................

1.3.3 Combined FEM/DEM Approach ................................................... 8

1.4 Hydraulic Fracturing, Proppants, Materials and Test Procedures .................... 9

1.5 Present Research Focus .............................................................................. 13

2 ANALYSIS OF UNIFORMLY SIZED PARTICLES ........................................ 15

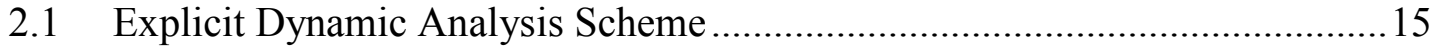

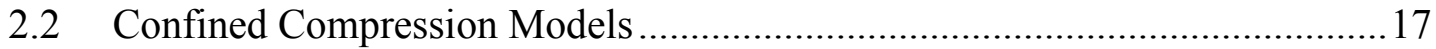

2.2.1 Results and Discussion...............................................................20

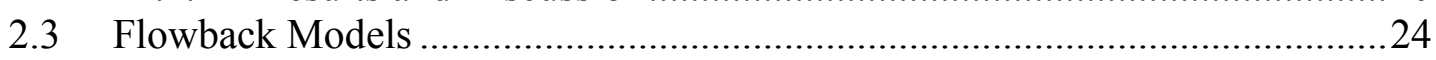

2.3.1 Results and Discussion.......................................................28

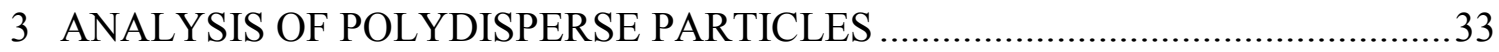

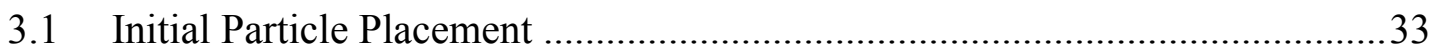

3.1.1 Literature Survey on Pack Configuration........................................33

3.1.2 Generating a Polydisperse Pack ..................................................... 34

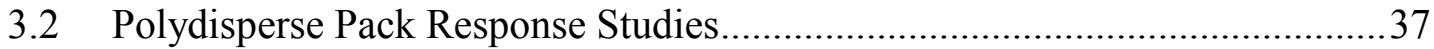

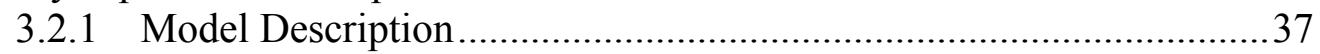

3.2.2 Results and Discussion..........................................................4 43

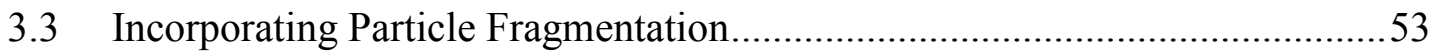

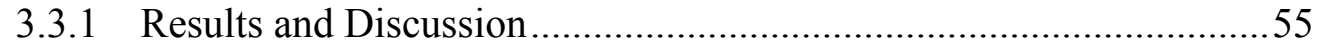

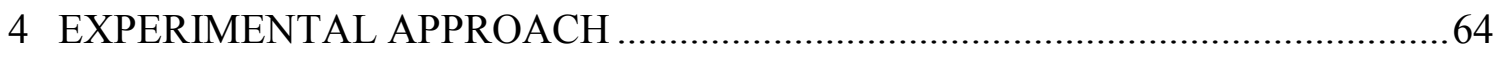




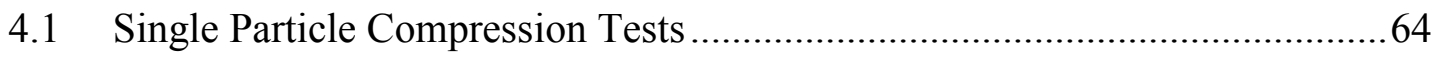

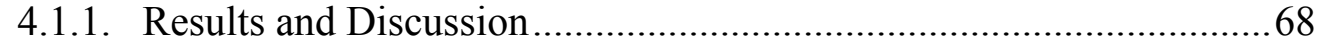

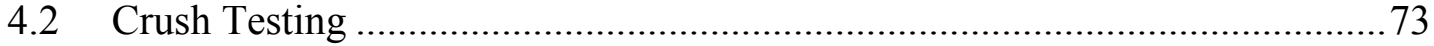

4.2.1 Equipment and Materials ............................................................. 73

4.2.2 Test Procedure ........................................................................... 75

4.2.3 Results and Discussion: LWC Mixtures ........................................76

4.2.4 Results and Discussion: HSP Mixtures ......................................... 90

4.2.5 Results and Discussion: Ottawa Sand Mixtures..............................93

5 COMPARISON OF EXPERIMENTS AND MODELS: CONFINED

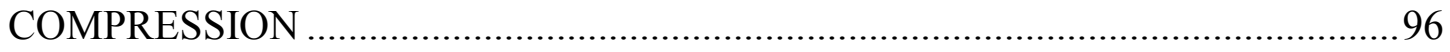

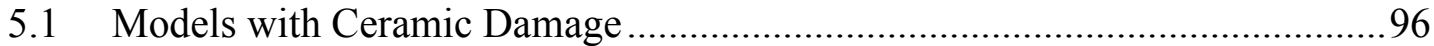

5.2 Models with Ceramic Fracture $-10 \%$ and 25\% Walnut................................ 97

5.3 Models with Ceramic Fracture - 25\% Pure Al ............................................ 101

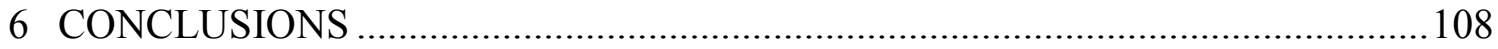

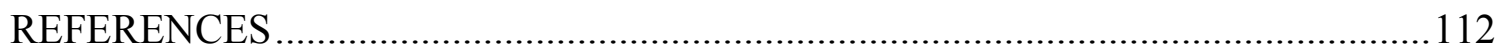

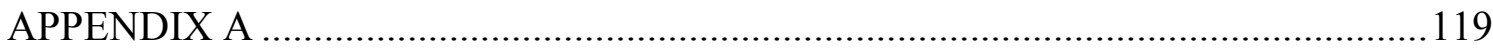

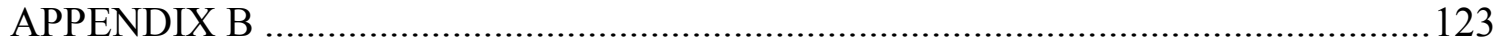

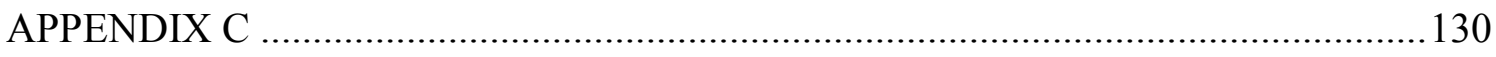

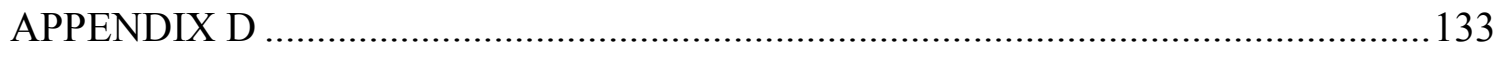

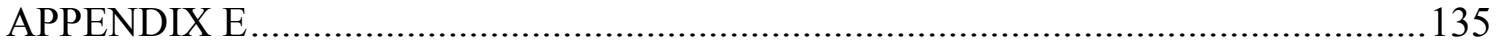

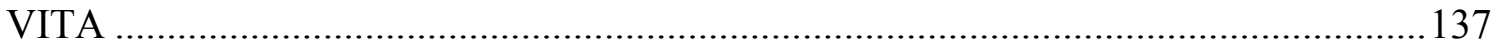




\section{LIST OF FIGURES}

FIGURE

Page

Fig. 1.1. Spherical proppants supporting an open hydraulically induced fracture [1].

Fig. 1.2. Photograph of mixture of large soft particles and small hard particles [10]

Fig. 2.1. Schematic of confined compression model and boundary conditions

Fig. 2.2. The load vs displacement response of the three walnut-ceramic mixture models

Fig. 2.3. Von Mises stress contour for $22 \%$ walnut model at $0.75 \mathrm{~mm}$ platen displacement.

Fig. 2.4. Von Mises stress contour for $30 \%$ walnut model at $0.75 \mathrm{~mm}$ platen displacement.

Fig. 2.5. Equivalent plastic strain (PEEQ) contour for $22 \%$ walnut model at $0.75 \mathrm{~mm}$ platen displacement

Fig. 2.6. Equivalent plastic strain (PEEQ) contour for 30\% walnut model at $0.75 \mathrm{~mm}$ platen displacement

Fig. 2.7. Flowback model with $15 \%$ walnut particles (blue) by weight. 26

Fig. 2.8. (a) First step of flowback loading, confined compression, (b) removal of side platen and (c) application of transverse load resulting in unstable particles moving out of the pack

Fig. 2.9. Transverse nodal velocities: $15 \%$ walnut model at $48 \mathrm{MPa}$ pressure for $\mu=0.3$ (a) linear elastic rock and (b) elastic-plastic rock

Fig. 2.10. Transverse nodal velocities: $15 \%$ walnut model at $48 \mathrm{MPa}$ pressure for $\mu=0$, (a) linear elastic rock and (b) elastic-plastic rock 30

Fig. 2.11. Transverse nodal velocities: $15 \%$ walnut model at $96 \mathrm{MPa}$ pressure for $\mu=0$ and elastic-plastic rock response 
Fig. 2.12. Transverse nodal velocities: $100 \%$ ceramic pack at $48 \mathrm{MPa}$ pressure for $\mu=0.3$ with elastic-plastic rock

Fig. 2.13. Transverse nodal velocities: $100 \%$ ceramic pack at $96 \mathrm{MPa}$ pressure for $\mu=0$ and elastic-plastic rock

Fig. 3.1. Models with 100 particles (a) and 150 particles (b) showing the initial low density particle configuration

Fig. 3.2. (a) Loose particle configuration with a rectangular domain (b) Particle configuration at the end of free fall (c) Imported particles in new analysis at the end of compressive loading

Fig. 3.3. Type-A mixture, 10\% walnut shell by weight: (a) Randomly generated polydisperse particles (b) particles at the end of free fall

Fig. 3.4. Particle configuration after free fall for $25 \%$ pure Al, Type-B mixture

Fig. 3.5. Particle configuration after free fall for $6 \%$ walnut shell, Type-A mixture

Fig. 3.6. Particle configuration after free fall for $18 \%$ walnut shell, Type-A mixture

Fig. 3.7. Particle configuration after free fall for $25 \%$ walnut shell, Type-A mixture

Fig. 3.8. Particle configuration at the end of free fall for the $25 \%$ by weight walnut particles mixture (light colored - walnut, dark shade - ceramic), 850 particles pack thickness $11 \mathrm{~mm}$, width $36 \mathrm{~mm}$.

Fig. 3.9. Type-A mixtures, $\mu=0.3$, Pressure vs displacement.

Fig. 3.10. Von Mises stress contour for $10 \%$ walnut - ceramic mixture at (a) $0.18 \mathrm{MPa}$ pressure (b) $3.2 \mathrm{MPa}$ pressure and (c) $9.4 \mathrm{MPa}$ pressure .45

Fig. 3.11. Von Mises stress contour for $25 \%$ walnut - ceramic mixture at (a) $0.18 \mathrm{MPa}$ pressure (b) $3.2 \mathrm{MPa}$ pressure and (c) $9.4 \mathrm{MPa}$ pressure .46

Fig. 3.12. Type-A mixtures, $\mu=0.3$, Pressure vs displacement after preload 47 
FIGURE

Page

Fig. 3.13. 10\% walnut mixture at $57 \mathrm{MPa}$ pressure to calculate void fraction (a) Deformed configuration and (b) processed image .48

Fig. 3.14. Type-A mixtures, Pressure vs void fraction .48

Fig. 3.15. 25\% walnut at $54 \mathrm{MPa}$ pressure (a) von Mises stress and (b) Equivalent plastic strain.

Fig. 3.16. Pressure vs void fraction for Type-B mixture, Al-pure and Al-alloy 50

Fig. 3.17. Von Mises stress for $25 \%$ by weight Type-B mixture at $57 \mathrm{MPa}$ pressure (a) 0.091 void fraction, $\mu=0.3$ and (b) 0.063 void fraction, $\mu=0.03$

Fig. 3.18. Von Mises stress contour at $58 \mathrm{MPa}$ stress for the 850 particle, $25 \%$ walnut model

Fig. 3.19. Pressure vs Non dimensional displacement plot comparing Type-A, $25 \%$ by weight walnut response for 400 and 850 particle models

Fig. 3.20. Pressure vs Non dimensional displacement plot comparing Type-A, $25 \%$ by weight walnut response for 0.4 and $0.8 \%$ failure strain, brittle cracking model

Fig. 3.21. Pressure vs Non dimensional displacement plot for Type-A, 25\% by weight walnut, plastic damage and brittle cracking model comparison

Fig. 3.22. Configuration of $25 \%$ Walnut, Type-A model at (a) $30 \mathrm{MPa}$ pressure and (b) $38 \mathrm{MPa}$ pressure. 58

Fig. 3.23. Pressure vs Non dimensional displacement plot for Type-A, $10 \%$ by weight walnut, plastic damage and brittle cracking model comparison

Fig. 3.24. Pressure vs Non dimensional displacement plot for Type-B, 25\% by weight pure $\mathrm{Al}$, plastic damage and brittle cracking model comparison

Fig. 3.25. Comparison of $10 \%$ and $25 \%$ walnut mixtures with fracture

Fig. 3.26. Comparison of element status at $25 \mathrm{MPa}$ pressure for (a) $10 \%$ walnut ceramic mixture and (b) $25 \%$ walnut ceramic mixtures 
FIGURE

Page

Fig. 3.27. Comparison of element status at $30 \mathrm{MPa}$ pressure for (a) $10 \%$ walnut ceramic mixture and (b) $25 \%$ walnut ceramic mixtures

Fig. 3.28. Comparison of 25\% Type-A and Type-B mixtures with fracture.

Fig. 3.29. Comparison of element status at $48 \mathrm{MPa}$ pressure for (a) $25 \%$ pure $\mathrm{Al}$ ceramic mixture and (b) $25 \%$ walnut - ceramic mixtures

Fig. 4.1. (a) LWC ceramic proppant and (b) HSP proppant prior to test with the datum sphere 66

Fig. 4.2. (a) Walnut proppant with the datum particle highlighting the thickness

(b) Same particle along the cross-section highlighting the area.

Fig. 4.3. (a) Pistachio proppant with the datum particle highlighting the thickness

(b) Same particle along the cross-section highlighting the area.....

Fig. 4.4. Effective stress vs Effective strain response for single particle compression of coated walnut (maroon) and coated pistachio (blue)

Fig. 4.5. (a) Load vs Displacement response for 12 of the 36 LWC ceramic particles (b) Load vs Displacement for a single particle highlighting the peak load at failure and drop in load after fracture

Fig. 4.6. $\ln \left(\ln \left(1 /\left(1-\mathrm{Pf}_{\mathrm{i}}\right)\right)\right)$ vs $\ln \left(\sigma_{\mathrm{f}}\right)$ plot with a linear fit to obtain Weibull modulus for LWC proppants....

Fig. 4.7. Load vs Displacement response for 12 of the 33 HSP ceramic particles....... 71

Fig. 4.8. $\ln \left(\ln \left(1 /\left(1-\mathrm{Pf}_{\mathrm{i}}\right)\right)\right)$ vs $\ln \left(\sigma_{\mathrm{f}}\right)$ plot with a linear fit to obtain Weibull modulus for HSP proppant particles

Fig. 4.9. Crush cell and loading piston mounted on the load frame 75

Fig. 4.10. Pressure vs non dimensional displacement LWC ceramic baseline. 77

Fig. 4.11. Walnut - Ceramic mixture (a) Crush as a function of weight percent of soft particles (b) Effective stiffness as a function of weight percent of soft particles and (c) Pressure vs non dimensional displacement 
Fig. 4.12. Walnut - Ceramic and Pistachio - Ceramic mixtures (a) Average percent crushes (b) Average stiffness (c) Walnut - Ceramic mixture image and (d) Pistachio - Ceramic mixture image

Fig. 4.13. Walnut - Ceramic and Al needles - Ceramic mixture (a) Average percent crushes (b) Average effective stiffness and (c) Al needles ceramic mixture image

Fig. 4.14. Al pellets - Ceramic and Al needles - Ceramic mixtures (a) Average percent crushes (b) Average effective stiffness and (c) Al pellets ceramic mixture image

Fig. 4.15. (a) Comparisons of average crush for all mixtures (b) Comparison of average stiffness for all mixtures

Fig. 4.16. Pressure vs non dimensional displacement Al Needles - HSP mixture . .92

Fig. 4.17. Pressure vs non dimensional displacement baseline, 10\% walnut and $10 \%$ pistachio - Ottawa sand mixture

Fig. 5.1. Pressure vs non-dimensional displacement comparison of Type-A models with damage and confine compression/crush tests

Fig. 5.2. Pressure vs non-dimensional displacement comparison of $10 \%$ and $25 \%$ walnut models with fracture and confine compression/crush tests .98

Fig. 5.3. Initial configuration prior to compressive loading for (a) $25 \%$ walnut LWC ceramic model and (b) 10\% walnut - LWC ceramic model

Fig. 5.4. Pressure vs non-dimensional porosity comparison of $10 \%$ and $25 \%$ walnut models with fracture.

Fig. 5.5. Von Mises stress contours at $33 \mathrm{MPa}$ pressure for (a) 10\% walnut model with ceramic fracture and (b) $25 \%$ walnut model with ceramic fracture

Fig. 5.6. Initial configuration for the three $25 \%$ pure Al - LWC ceramic models (a) large needles (b) small needles and (c) pellets. 102

Fig. 5.7. Pressure vs displacement response of $25 \%$ pure Al models, comparison to test results for $25 \%$ pure Al needles 
FIGURE Page

Fig. 5.8. Pressure vs porosity comparison for $25 \%$ pure $\mathrm{Al}$ models and $25 \%$

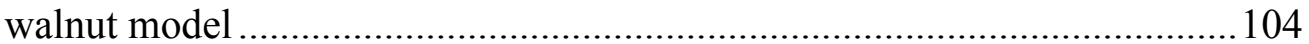

Fig. 5.9. Von Mises stress contours at $38 \mathrm{MPa}$ pressure for (a) 25\% pure Al large needles model (b) $25 \%$ pure Al small needles model and (c) $25 \%$ pure Al pellets model. 104

Fig. 5.10. Von Mises stress contour at $38 \mathrm{MPa}$ pressure for $25 \%$ walnut model ......... 105

Fig. 5.11. Pressure vs non-dimensional displacement comparison of $25 \%$ pure Al large needle, 25\% walnut model with test data. 106 


\section{LIST OF TABLES}

TABLE

Page

Table. 2.1. Force, Void Fraction and total particle mass for the three walnut-ceramic mixtures at platen displacement of $0.75 \mathrm{~mm}$ .22

Table. 3.1. Material properties and particle description ...................................... 40

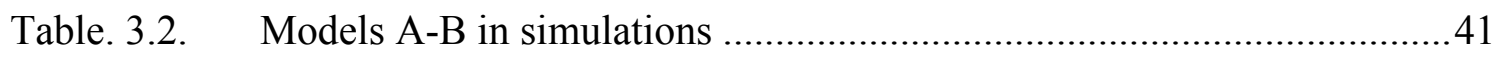

Table. 4.1. Material, size range and concentration of deformable particles considered in mixtures. The base material is LWC ceramic proppant. Total sample weight is $41.15 \mathrm{~g}$....................................... 75

Table. 4.2. Crush and Stiffness data for walnut ceramic mixture ............................ 78

Table. 4.3. Crush and Stiffness data for Al needles ceramic mixture ......................78

Table. 4.4. Crush and Stiffness data for Al pellets ceramic mixture........................79

Table. 4.5. Crush and Stiffness data for pistachio ceramic mixture.........................79

Table. 4.6. Crush and Stiffness data for HSP with Al needle mixture ......................91

Table. 4.7. Crush and stiffness data for HSP with Al sphere mixture ......................92

Table. 4.8. Average crush and stiffness comparison of Al needles and Al spheres mixtures with LWC and HSP proppants

Table. 4.9. Average crush and stiffness of baseline sand and 10\% walnut and pistachio mixtures, also compared with LWC mixtures 


\section{INTRODUCTION}

\subsection{Overview}

Granular materials are a conglomeration of discrete solid particles. They are large enough so that they do not exhibit Brownian motion. Their application areas range from geophysics, pharmacy, oil \& gas, powder metallurgy, polymer technology, casting technology, agriculture, construction technology etc. Proppants are a special application of granular materials employed in oil/gas well stimulation.

Hydraulic fracturing is a process where a highly pressurized fluid is pumped into an oil/gas well at sufficiently high rates to create fractures. Proppants are small granules which are delivered to these fractures to ensure that the flow paths remain open against the rock pressure. Proppants settle in either as a closed pack arrangement or as a single layer while ensuring sufficient permeability to enable continued/enhanced oil/gas production [1]. Historically sand has been the most commonly used proppant material. However as the well depths increase, the pressures increase. Sand is unable to resist the high pressures and crushes generating free fines (fragmented pieces of sand particles) which reduce permeability by occupying the pore space in the pack [2]. At higher well depths synthetic proppant, generally sintered bauxite or ceramic is employed. Current research in the industry is focused on employing ultra light weight material as proppants. Some of the different materials considered include polymers, biomaterials like walnut shell or pistachio shells and hollow ceramic proppants [3, 4]. Research is also focused on employing a mix of soft and hard particles e.g. walnut/polymer particles with sand or aluminum fibrils/aluminum pellets with ceramic with the intention of improving the pack stability [5].

This dissertation follows the style and format of Composites Science and Technology. 
Currently according to the industrial practices proppant characterization involves long term and expensive conductivity tests [6]. That apart the mechanics governing the proppant pack response, in particular the effects of particle fracture and polydispersity on the pack porosity are not understood clearly from the tests.

In the research presented here computational models have been developed to model hard and soft proppant mixtures. The finite discrete approach is employed to better understand the mechanics governing a proppant pack and help develop a virtual parametric test bed capability to screen proppant samples. To this end models have been developed in the software ABAQUS 6.8.3 using the explicit time integration scheme. The models simulate the load conditions to which proppants are exposed to in a hydraulic fracture. Proppant mixtures are modeled to take into account polydispersity in size, shape and material properties. Single particle compression tests on individual particles have been carried out to obtain mechanical properties to be incorporated in the models. Compression tests on proppant mixtures have been carried out according to industry standards $[7,8]$. The load vs displacement response of the computational models has been compared to the compression tests. This enables us to better understand the mechanics governing the pack response and also helps in improving/refining the modeling approach.

In this section next a brief discussion on granular materials and their typical/unique properties is provided. Further, the computational techniques which are employed to analyze granular materials are discussed. This is followed by an introduction to proppants, their different material types, loading conditions and test procedures employed to characterize them.

\subsection{Granular Materials}

Granular materials refer to class of materials with size greater than one micron. Above this limit particles do not exhibit Brownian motion and physics of motion is primarily dependent on external forces and particle interactions and not on thermal 
agitations [9]. They can be either dry or wet and can be encountered as either particles or powders. Granular materials are typical as they exhibit behavior which is akin to solids, liquids and gases. They can withstand shear loads and form heaps, they flow with a boundary layer and they also exhibit compressibility [10]. These typical behavioral patterns make it extremely difficult to predict the behavior of granular materials.

Granular heaps are distinct from liquids as they remain stationary until their slope exceeds a specific angle, known as the angle of repose. Beyond this slope grains begin to flow. This flow is what happens in case of an avalanche. It is observed that only the top few layers move while the bulk of the heap remains stationary like a solid. Thus a boundary layer is observed in case of the flow of granular materials [11, 12].

An example of an unusual behavior of granular material is its ability to transmit stresses perpendicular to the direction of loading [11, 12]. When a pack of granules is compressed it transmits load through interparticle contacts. The contacts keep on changing based on changes in particle position and particle deformation, resulting in a non uniform stress distribution in a granular pack in contrast to a homogeneous solid. This transfer of loads due to contact results in a vertical load being transmitted along the horizontal direction. In case of fluids the pressure of fluid in a container is dependent on the height of the fluid column. The transverse load transfer in case of granular materials results in a divergence from this fluid behavior and is generally observed in sufficiently tall vessels. The friction between the wall and the particles is sufficient to withstand the load. The interparticle load transfer and friction also result in a phenomenon known as arching. It is the same phenomenon which enables the construction of arches on cathedrals with the appropriate placement of a keystone. Arches occur naturally in case of granular materials and the force between particles provides stability. Such arches are one of the major problems with regards to material handling applications e.g. silos and hoppers [13]. Choking is observed while emptying of silos due to the formation of arches. Conversely for the proppants a stable arch has been found to be essential for preventing flowback and maintaining a stable pack $[14,15]$. Also the linear relation 
between the filling height and draining time in case of an hourglass filled with fine sand is due to interparticle friction.

The unique aspects of granular materials can be described within the context of nonlinearity, dilatancy and compressibility. The overall response of granular materials is characterized by particle movement and particle deformation. Particle movement is an irrecoverable phenomenon. The load is transferred in granular materials via contact which obeys the Hertz's law of contact and describes a nonlinear relationship between load and particle deformation due to a continuous change in the contact patch area [16]. The combination of these two phenomenon results in a granular bed exhibiting nonlinear response to loading. A granule bed as it is compressed tends to show a stiffening response. The dilatancy principle, which was developed by Osborne Reynolds in 1885 is an important phenomenon observed in granular materials. Accordingly, on the application of compressive loads granular materials expand. This is in particular observed with strongly compacted materials while in the case of loose materials a compressive load causes shrinkage in volume [11]. Another important factor with regards to granular materials is segregation. The Brazil nut effect is the most commonly mentioned phenomenon. On being excited the particles are arranged in a way such that the largest particles are transported to the top of the heap while the smallest particles settle to the bottom. The arrangement is such which reduces the potential energy of a heterogeneous pile. The segregation occurs due to vibrations or due to shearing which is experimentally simulated in a horizontally rotating drum half filled with granular materials.

A proper understanding of granular material behavior is important due to the wide range of applications. Powder processing is important for pharmaceutical companies. Large manipulation of sand, gravel, cement etc is necessary for construction. Agriculture and food processing requires transport and processing of bulk commodities like grains, flour. Also land tilling involves manipulation of granular materials. The design of the equipments employed for material handling in the above mentioned applications is dependent on the flow and stress equilibrium properties of granular media 
$[9,11,13]$. Granular materials also provide vibration isolation or shock absorption [12]. This apart geological processes like avalanches, landslides and even plate tectonics can be considered as effects of granular materials. The interaction of granular media in silos or in confined places is fraught with the dangers of explosions due to electrostatics wherein charge is generated due to particle impact causing sparks [9].

\subsection{Computational Techniques to Model Granular Materials}

Computational techniques employed to analyze granular materials can be categorized into two broad categories one is continuum approach and the other is discrete approach. Phenomenological continuum models utilize finite element modeling, the constitutive material descriptions is obtained from testing. The discrete approach is particularly useful as it allows each particle to be modeled individually. The interaction of particles can then enable the observation of the nonlinear stress-strain response, failure envelopes, and transition from brittle to ductile behavior of the granular pack it can also capture local particle failure and shear bands [17]. The most commonly employed discrete technique is the discrete element method (DEM). The finite discrete approach combines the continuum mechanics approach with the discrete element formulation. In this approach like the discrete approach each particle is modeled individually but unlike the DEM approach particle deformation is captured by meshing it with finite elements [18].

\subsubsection{Continuum Approach}

In this approach a granular pack is considered as a porous solid and studied as a continuum. This technique is employed in soil mechanics and geomechanics which deal with the problem of soil compression, shear and stability coupled with fluid flow. Coupled differential equations are employed which simulate the solid fluid motion. These equations are then solved numerically in a finite element code which can be used to study problems of soil mechanics [19]. This model is much more economical to solve 
compared to the discrete model which involves solution of a number of complex contact interaction problems. The governing differential equations can be derived using two approaches, first is by considering mixture theory and second is a physical approach. The physical/phenomenological approach considers all the interactions between the solid and liquid phase. In case of the physical approach any nonlinearity is specified at the skeleton. Here skeleton refers to the solid structure formed due to interacting granules. In case of porous media theory which covers the above mentioned approaches, interaction is considered between this solid skeleton and the fluid present in the porosities. The effective stress is considered where the effective compressive stress is the external stress minus the pore fluid pressure.

To specify the nonlinearity requires number of tests on the dry skeleton i.e. no pore fluid. Some of these include confined compression, box shear [20] and triaxial tests $[20,21]$. The confined compression essentially provides us with a compressive response while the triaxial tests provide us with a combined compression and shear response. The data from these tests needs to be analyzed to obtain the coefficients defining the plasticity in case of soils or any other granular material [22]. A number of plasticity theories are available for soil mechanics e.g. cam-clay, Drucker-Prager cap plasticity [19, 23]. Such an approach has been employed to model a proppant layer and fracture was modeled using only 800 elements. The model also included nonlinear mechanical properties of the proppant layer which were determined by carrying out triaxial tests. Also by considering a strain to failure criteria flowback was also simulated [24].

In case of the theory of porous media certain phenomenon like shear bands are highly dependent on the mesh density. To overcome this problem the micropolar Cosserat theory is employed which considers the angular rotations of the particles and also includes the particle orientation influence through the use of fabric tensor [25].

Reproduction of complex behavior with continuum methods requires complex constitutive models containing many parameters and variables [17]. Also the local damage like shear band or particle fragmentation which can be observed through discrete formulation is difficult to capture through a continuum model which uses a 
finite element mesh. One more issue with the use of this model is its applicability to narrow regions. Generally continuum approach is valid when the grain dimensions are much smaller compared to volume of interest. In case of the proppant problem for a stable pack the thickness of the granular pile is limited to $\sim 5-6$ particles [14]. Thus the applicability of continuum approach is suspect in this case.

\subsubsection{Discrete Approach}

Broadly the discrete approach can be divided into two approaches, the soft sphere and the hard sphere approach. The approach known as the hard-sphere approximation forms the basis of the collisional or the event-driven (ED) models. It is also the principle behind methods like the Monte Carlo and steepest descent [11]. In case of hard spheres (hard implies no particle deformation and interpenetration) the loss of linear momentum is characterized only on the bases of coefficient of elastic restitution, when rotations are neglected. In these models restitution of elastic energy and friction are completely decoupled. Dry friction is modeled using Coulomb's friction laws. Monte Carlo method has been employed extensively to model particle segregation. A particle packing is obtained by considering the minimization of the potential energy of the packing $[26,27]$.

In case of the soft-sphere approximation friction and restitution come into picture when spheres penetrate each other. The magnitude of contact forces is dependent on the depth of penetration. Algorithms like molecular dynamics (MD) or distinct element methods (DEM) are covered under soft spheres. In these models the important parameters are, duration of contact and particle interpenetration. The basic principle is to solve the equations of change in linear and angular momentum in regular increments. DEM was originally developed by Cundall for rock mechanics problems and later employed for granular materials by Cundall and Strack [17]. The algorithm of DEM is based on conditionally stable explicit time marching scheme and does not require the inversion of the stiffness matrix. The effectiveness of DEM is with its capability to model contact interaction between large numbers of particles [28]. In the classical DEM 
the particles are rigid spheres. The normal contact forces and tangential slip conditions are modeled using a combination of springs and dashpots $[11,25]$. The classical DEM models are unable to model particle rolling and hence cannot model the shear strength of granular soil. Also spherical particles cannot take into account the effect of angularity of soil particles. Several methods have been proposed to model particle angularity [29]. The contact forces between particles are modeled using springs and dashpots and hence to model complicated particle material properties is difficult. DEM is employed to model quasi-static deformation of soils/ granular materials, e.g. triaxial tests, confined compression and box shear tests [30-32]. It is also employed to study aspects like particle packing, and dynamic problems like flow of granular media through hoppers or conveyer systems [33-36].

\subsubsection{Combined FEM/DEM Approach}

In a number of cases, in particular if the granules are made of highly deformable material having a complex nonlinear relationship the overall response of the particle bed will significantly differ if the particles are modeled as rigid or linear elastic as is the case with the previously discussed ED and DEM approach. Similarly particle angularity can also give significantly different results compared to simulations if particles are modeled as spheres. As stated previously it is difficult to model such aspects in case of DEM. Munjiza proposed the combined FEM/DEM technique wherein the particles are modeled as a continuum while the contact detections are carried out based on the DEM code [18]. Because the particles are modeled as a continuum it is possible to introduce large deformation, and fracture in the particles. This method has been applied extensively to model powder compaction [37-39]. The dynamic explicit finite element codes like ABAQUS and LS-Dyna have been employed to model multiple particle interactions. These simulations employ the general contact capability which greatly simplifies the definition of contact surfaces. Zavaliangos modeled powder compaction using ABAQUS [40]. The tensile stresses developed in particles which can result into their 
fracture were observed from the simulations. The influence of co-ordination number i.e. number of surrounding particles for a given particle on its tensile stress distribution is observed [41]. Furthermore the particles were also subjected to triaxial loading conditions and simulation results were used to develop a cap-plasticity model for the particles [42]. Zhang modeled particle interaction with a mixture of hard and $40 \%$ soft

particles [43]. Choi and Gethin modeled powder compaction considering polydispersity and different particle shapes. They also considered the effect of shear load from the platen on the response of the pack and the influence of platen surface roughness [44]. Kabir et el modeled particle flow in a shear cell using explicit FEM and compared the results with DEM code $[45,46]$. The drawback with the combined FEM/DEM approach is the requirement of significant computational resources which has limited the application to mostly 2D problems, though 3D problems have also been considered [47, 48].

\subsection{Hydraulic Fracturing, Proppants, Materials and Test Procedures}

Hydraulic fracturing is employed to enhance the productivity of an oil/gas well by creating fractures in the rock which create highly conductive fluid flow paths and increase the pressure gradient between the well bore and the porous rock. Hydraulic fracturing is a part of well stimulation process and the fractures are created when highly pressurized fluid is pumped in a well at sufficiently high rates. These fractures provide flow paths for oil and orient radially away from the well bores. When the pressure from the fracturing fluids is reduced to begin oil/gas production the fractures tend to close under the influence of rock pressures known as closure stresses. Proppants are mixed with the fracturing fluid and are delivered to these fractures to ensure that the flow paths remain open while resisting the rock pressure. Proppants settle in the fractures forming a granular pack and prop them open against the closure stresses. The porosity within the packed particles ensures sufficient permeability to enable continued/enhanced oil/gas production $[1,2]$. A schematic of proppant supporting a fracture is shown in Fig 1.1. In 
a hydraulic fracture proppants can be packed either as a single layer (Fig 1.1, right) which is termed as a partial monolayer or a full granular pack (Fig 1.1, left) [3, 4].

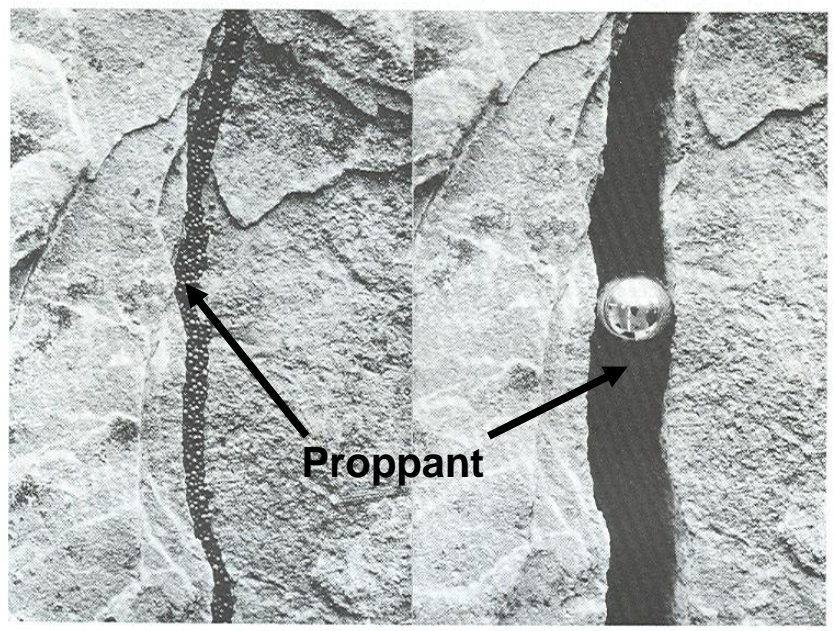

Fig. 1.1. Spherical proppants supporting an open hydraulically induced fracture [1]

Hydraulic fracturing employing proppants was introduced in late 1940's. The first ceramic proppants were sintered bauxite introduced in 1976 [1]. From strength perspective proppants are classified as low strength, intermediate strength and high strength. Low strength materials typically include natural quartz sand and can be used at closure stress lower than 5000 psi. Intermediate strength proppant materials include alumina silicate (ceramic) proppants which can be applied at pressures of 10000 psi. High strength ceramic proppants typically include sintered bauxite and zirconia silicate proppant; these are employed up to pressures ranging from 15000-20000 psi. Materials like sand and alumina silicate proppants have specific gravity of 2.6 and are classified as light weight. Sintered bauxite proppants are high density proppants with a specific gravity of 3.6 [1]. Beyond its range of applicability ceramic or sand proppants fracture into multiple pieces and that results in loss of load bearing capacity as well as reduction in the available pore space. Polymer coating is applied on the proppants to reduce the 
stress concentration at particle contacts. Resin coated sand can withstand up to 10000 psi closure stresses, resin coated sintered bauxite proppants can withstand pressures up to 30000 psi [1]. Hollow ceramic proppants with a specific gravity of 1.75 or proppants utilizing polymer or biomaterials like walnut shells or pistachio shells with an even lower density of 1.25 are termed as ultra light weight proppants [3].

Ultra light weight particles like resin coated ground walnut shell, pistachio shells or tailored polymers are generally employed with a partial monolayer [3, 4]. When a full granular pack is simulated with only light weight particles they tend to deform significantly and result into a loss of pack porosity, hence a full proppant pack generally employs hard particles like sand or ceramic. The light weight softer particles are mixed with the hard particles in the full pack with an intention of enhancing the pack stability [49-51]. The harder particles are generally smaller than the soft particles e.g. the ceramic or sand particles generally fall within a 20/40 mesh size $(0.4-0.8 \mathrm{~mm})$ while the walnut shell particles in Fig. 1.2 have a size within the 16/30 mesh size (0.6-1.2 mm) [5]. Furthermore the softer proppants also have different shapes compared to the almost spherical ceramic particles, e.g. needle like shape of aluminium compared to a more rounded walnut particle. Commercial products also include chopped fibers or thermoplastic sheets as the softer proppants [51].

During oil/gas production a pack of proppants is subjected to two major forces one is the compression coming from the rock faces this is due to the depth at which the fracture occurs and second is the transverse shear force on the pack due to pressure gradient in the fracture and the shear drag force exerted by the flowing fluid. The compressive forces tend to crush the proppants reducing the porosity available for fluid flow. The transverse forces tend to dislodge the proppants from the pack, these free proppants flow with the fluid to the surface and are termed as the back produced proppants. Proppant back production presents a safety hazard as it may result into erosion of well and surface facilities. Also remaining proppants in a wellbore can shut off production. Costly and manpower intensive surface handling procedure is necessary to handle proppant flowback [52]. 

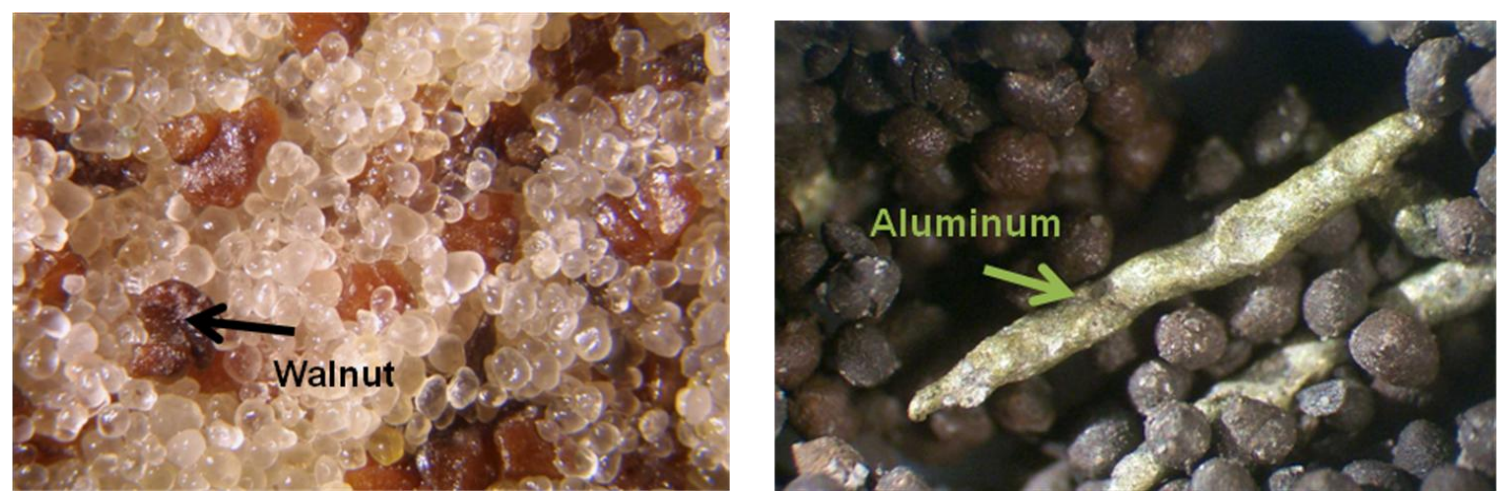

Fig. 1.2. Photograph of mixture of large soft particles and small hard particles [10]

The characterization of proppants involves multiple tests including single particle compression, crush analysis tests, sieve analysis, sphericity and roundness characterization particle settlement and conductivity tests $[3,4,6]$. The crush analysis test measures the percentage of proppant fracturing under confined compression loading condition. This is an indicator of the load bearing capacity of a proppant pack. For a given load if more than $10 \%$ by weight proppant particles get fractured then the proppant is rejected. According to the current standards the crush test is performed dry and at room temperature. This test has been employed to study the crush resistance of polymer-sand mixtures. It has been reported that addition of softer particles improves crush resistance while the pack stiffness reduces [53]. Effect of stress cycling on crush resistance of a pack has also been studied and improvement has been reported with the mixing of polymer particles with sand [54]. Currently the crush test procedure does not account for pack porosity, though modification to the crush cell so that fluid flow through the proppant pack can be measured to estimate permeability have been carried out [55]. Similarly efforts have also been ongoing to modify the crush tests to account for temperature and moisture effects on crush resistance of different proppants [56].

The long term conductivity tests are conducted with an aim of studying fluid flow through a proppant pack under compression $[6,57]$. These tests are carried out in a 
specially designed flow cell. The proppant is placed between two rock platens in the cell and pressure is applied gradually, temperature can also be controlled. Often the pack is allowed to stabilize at a particular pressure for 48 hours. The rate of fluid flow though the pack as a function of time and pressure, pressure gradient along the flow direction and distance between the two platens are the primary parameters which are measured. The influence of addition of softer particles to a proppant pack on pack permeability has been studied extensively and a reduction in the flow rate has been observed [49-52]. Research efforts are also on to study the influence of chemical reactions between the fracturing fluid and the proppant which is known as proppant diagenesis on pack permeability [58]. A modification to the conductivity test wherein an arrangement is made to collect loose proppant and measure their flow rate is used to study proppant flowback. Several researches have focused on understanding the factors affecting proppant flowback and finding ways to reduce it [14, 15, 49-52]. Apart from the DEM (discrete element method) simulations by Asgian [14] the rest focus on experimental approach. Some factors which seem to aid reduce proppant back production are softer formation i.e. softer rock, more angular proppant, resin coated proppants wherein the resin cures once it settles in the fracture and binds particles together and addition of softer particles.

\subsection{Present Research Focus}

The computational modeling of granular packs is a very complex and a challenging field where considerable breakthroughs are needed to successfully predict response of a pack under dynamic conditions. From an overview of the literature on the discrete simulation of particles, most are concerned with quasi-static powder/granule compaction and do not consider polydispersity. Furthermore particles are considered to be elastic-plastic while the platen is taken as rigid. However in experiments there are many instances where the platen deformation mainly due to particle indentations plays a significant role in determining the effectiveness of a given granular material application. 
In our study, we take on select, unique aspects to understand the best practices in designing the proppant mixture consistent with its service conditions. The computational models are developed in coordination with small scale experiments such as single particle compression and crush tests to assists the characterization of pack response. This approach will facilitate the screening of proppant samples and reduce the current dependency on long term conductivity tests enabling.

This study is based on two different compositions of hard and soft particles, namely Type A and Type B. Type-A is a mixture of ceramic and walnut shell particles while Type-B is a mixture of ceramic and pure aluminum needles. The ceramic particles are the light weight alumina silicate which is commercially known as Econoprop. In the following Chapters 2 and 3, the rationale and the details of the analytical models will be presented. Specifically Chapter 2 is dedicated to uniform size particles of Type-A mixture to understand the confined compression and flowback load conditions. The platens are assigned with nonlinear rock material properties to consider the effect of particle indentation on pack stability and conductivity. Chapter 3 introduces particle size and shape as variables in both Type A and Type B compositions. The compressive response is described by the variation of void space in the pack as a function of applied load. The influence of particle material, shape and interparticle friction on compression response is demonstrated. Models are also developed to document the effect of particle fragmentation. The experiments of this study are fully described in Chapter 4. The single particle compression tests on walnut, pistachio and ceramic particles as well as the crush tests on Type-A and Type-B mixtures are discussed in detail. Finally in Chapter 5, a comparison of computational models and crush tests are examined. 


\section{ANALYSIS OF UNIFORMLY SIZED PARTICLES}

This section introduces the loading conditions and modeling approach employed to simulate proppant mixtures. Models with uniformly sized particles are discussed in this section. Models are analyzed using the combined finite/discrete element technique employing the commercial software ABAQUS v 6.8.3 explicit code. To begin with a short discussion on explicit time integration is provided. For the modeling approach three different compositions of walnut-ceramic mixtures have been modeled in confined compression. For the flowback models effect of rock material property, addition of softer particles and interparticle friction on flowback resistance is studied. The models discussed in this section are the first set of models developed and provide a base for the development of subsequent polydisperse models.

\subsection{Explicit Dynamic Analysis Scheme}

A detailed discussion on explicit dynamic analysis as employed in FEM is available in the ABAQUS 6.8.3 user's manual section 6.3.3 [23]. Application of this approach in the context of finite discrete element method is provided in, Munjiza [18]. Here a summary of the explicit dynamic scheme is provided.

Explicit dynamic analysis is computationally efficient for the modeling of highly dynamic events like impact, e.g. a car crash simulation. It is also employed with quasistatic analysis where complex contact conditions and highly nonlinear deformations persist. Due to these reasons the ABAQUS explicit code is employed in the current simulations rather than the implicit code. The explicit code uses the central difference time integration rule; this is a conditionally stable scheme and limits the time increment. A large number of small time increments are performed in this scheme. Compared to the implicit analysis the number of increments is significantly high. The advantage of this scheme comes from the fact that it does not require solution of a set of simultaneous equations. In the dynamic equilibrium equations the mass matrix is diagonalized, 
termed as the lumped mass matrix. Thus rather than considering the mass as distributed over the element, it is assumed to be concentrated at the nodes of the FEM mesh. With the use of a diagonal matrix, the inversion becomes simple to calculate. The explicit procedure also does not require any iterations to achieve convergence and no tangent stiffness matrix is needed. The accuracy of the scheme is controlled by the size of the time step. For the central difference time integration applied in the explicit scheme the nodal acceleration $(a)$ at time $t$, is computed by solving the dynamic equilibrium equation. The velocity $(v)$ at time $(\mathrm{t}+\Delta \mathrm{t} / 2)$ is computed based on acceleration at time $\mathrm{t}$. Further the nodal displacements $(u)$ at time $(\mathrm{t}+\Delta \mathrm{t})$ are computed based on the velocity at $(t+\Delta t / 2)$. In equation form this can be written as:

$$
\begin{aligned}
& a_{t}=M^{-1}\left(P_{t}-I_{t}\right) \\
& v_{t+\Delta t / 2}=v_{t=\Delta t / 2}+\frac{\left(\Delta t_{t+\Delta t}+\Delta t_{t}\right)}{2} a_{t} \\
& u_{t+\Delta t}=u_{t}+v_{t+\Delta t / 2} \Delta t_{t+\Delta t}
\end{aligned}
$$

Note that the first equation is the dynamic equilibrium equation where $M$ is the diagonalized mass matrix, $P_{t}$ is externally applied load vector at time $\mathrm{t}$ and $I_{t}$ is the internal force vector calculated from contact interactions and element deformation. The second equation calculates velocity at the next half time increment from acceleration at the current time. The third equation computes displacement at the next time increment based on the previously calculated velocity. Also note that this procedure is repeated for every degree of freedom present in the model.

As mentioned previously the central difference time integration scheme is conditionally stable. The stable time increment is limited by the highest frequency $\left(\omega_{\max }\right)$ of the system, equation 4 . An approximate stable time increment is given by the least time taken by a dilatational wave to traverse across an element in the mesh, equation 5. Here $L_{\min }$ is the smallest element dimension in the mesh and $c_{d}$ is the dilatational wave speed. $c_{d}$ is defined in terms of the elastic modulus $(E)$ and density $(\rho)$ 
of the material for a linear elastic isotropic material with zero Poisson's ratio, equation 6. The dilatational wave speed at current time increment is computed in ABAQUS explicit by calculating hypoelastic material moduli (effective Lame's constants) from the material constitutive response [23].

$$
\begin{aligned}
& \Delta t \leq \frac{2}{\omega_{\max }} \\
& \Delta t \approx \frac{L_{\min }}{c_{d}} \\
& c_{d}=\sqrt{\frac{E}{\rho}}
\end{aligned}
$$

In certain cases a few small elements in the mesh can significantly reduce the stable time increments. In such situations either mass scaling is employed which increases the density and reduces the value of the dilatational wave speed or the time period of the event can is reduced. This approach is employed in case of quasi-static analysis. Mass scaling remains the only option in cases where the material response is rate dependent and reducing the event duration could result in erroneous results. At the same time mass scaling can result into additional inertial effects, hence the kinetic energy needs to be monitored during the analysis. The kinetic energy with mass scaling should be less than $10 \%$ of the internal energy.

\subsection{Confined Compression Models}

Confined compression load case simulates proppants deep within a granular pack in a hydraulic fracture away from a well bore. In this condition proppant pack is being compressed between the two rock faces, at the same time its transverse motion i.e. perpendicular to compression direction is resisted by surrounding particles. A proppant crush test $[7,8]$ also simulates the same condition, here a given mass of proppant is 
compressed within a cylindrical crush cell and the transverse motion of the particles is resisted by the walls of the cylinder. The confined compression/ crush test is one of the most basic tests conducted on proppants to study the resistance of a proppant pack to compressive loading.

Herein we present models simulating confined compression with a combined finite/discrete element method employing ABAQUS 6.8.3 explicit code. Each particle is modeled individually and is meshed with finite elements. This approach enables us to introduce large deformation, and fracture in the particles. General contact capability is utilized to simulate inter-particle interaction. General contact is used as it significantly reduces the effort in defining contact regions. A detailed discussion on general contact and its method of application in the ABAQUS input file is provided in APPENDIX-A.

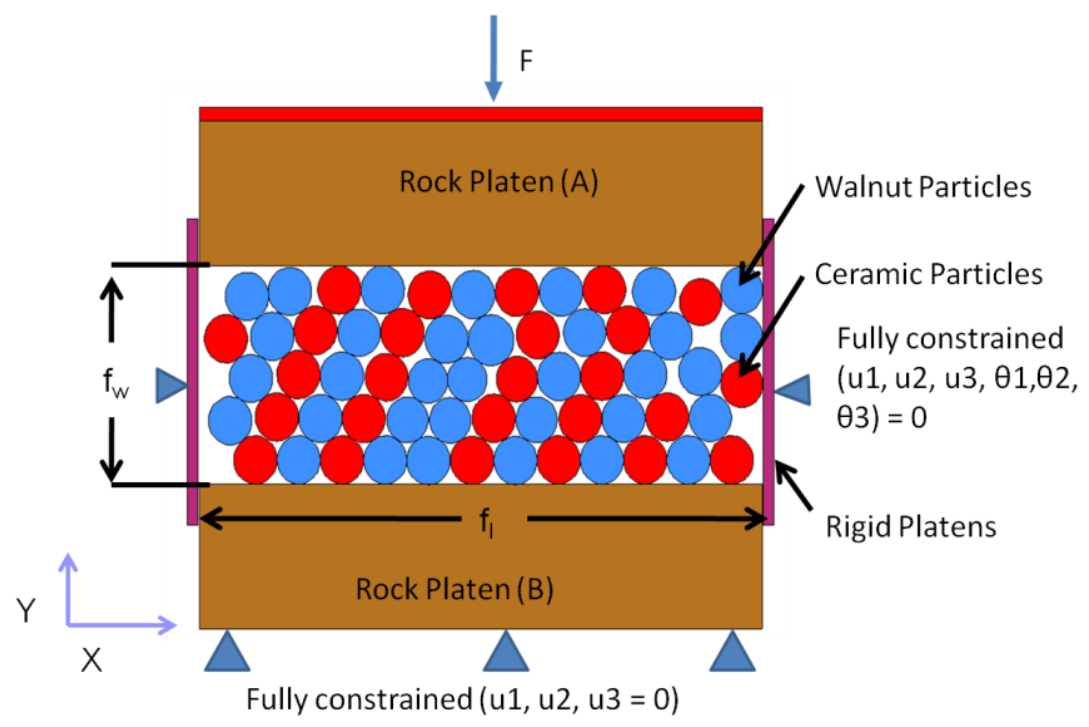

Fig. 2.1. Schematic of confined compression model and boundary conditions

This model consists of 60 particles placed between the two horizontal rock platens that represent the fracture width $\left(f_{w}\right)$ as shown in Fig. 2.1. The initial fracture width prior to the load application is $4.5 \mathrm{~mm}$. Two additional rigid platens are 
introduced as side enclosures. The distance between these two rigid platens represents the fracture length $\left(f_{1}\right)$ in the present model this is limited to $13 \mathrm{~mm}$. The lower rock platen (B) is constrained against any motion and the upper rock platen (A) is constrained to undergo motion in $\mathrm{Y}$-direction. The rock platen (A) consists of two layers where rigid elements are assigned to the top layer. The rock pressure is represented with prescribed platen (A) displacement. Maximum compressive displacement of $0.75 \mathrm{~mm}$ is introduced incrementally at the reference node of the rigid element layer of platen (A). The loading is quasi-static i.e. negligible inertial effects. Mass scaling is employed to speed up the calculations and inertia effects are monitored. The initial random packing of particles and the 3D mesh is generated with the pre-processor Hypermesh9.0. Three dimensional continuum elements, C3D8R, with eight nodes and reduced integration are used to model the particles and the rock platens. Each particle is modeled as a cylinder with a diameter of $1 \mathrm{~m}$. The model is simulated under plane strain condition by enforcing constraints along the cylinder axis preventing deformation of the cylinders along the axial (Z) direction. The number of elements in the model is 35476 and the number of nodes is 58782 .

Mixed pack of soft (walnut) and hard (ceramic) particles is considered with 15\%, $22 \%$ and $30 \%$ walnut particles by weight. The schematic in Fig. 2.1 represents a mixture with $30 \%$ walnut particles by weight. The specific gravity of ceramic and walnut is 3.6 and 1.25 respectively. Walnut is described with an elastic-plastic constitutive behavior obtained from single particle compression tests $[59,60]$. The elastic modulus of walnut is $3.7 \mathrm{GPa}$. From previously conducted tests walnut begins to undergo non-recoverable deformation at low compressive loads. In the current models walnuts are assumed to begin to undergo plastic deformation at low stress of $8 \mathrm{MPa}$, it continues to harden till it reaches a stress of $44 \mathrm{MPa}$. Ceramic is represented as a quasibrittle material where it remains linear-elastic until the tensile stresses reach its bending strength. At this stage micro-cracking is assumed to occur and at subsequent increments, the modulus is represented as $E=(1-d) E_{0} . E_{0}$ is the undamaged elastic modulus and $d$ is the scalar degradation variable which is defined as a part of material strain softening data 
in the concrete damage plasticity model in ABAQUS [23, 61], a further discussion on this model is provided in APPENDIX-B. Note that in the present model the ceramic material property is defined using the strain softening material data for concrete which is given in the ABAQUS example problem manual 6.8.3. Based on this the bending strength is defined as $29 \mathrm{MPa}$. The strain softening is defined based on the residual stress carrying capacity after partial cracking and the crack opening in terms of displacement. Accordingly the material loses its tensile load bearing capacity when the crack surfaces separate by a distance of $0.045 \mathrm{~mm}$. The rock platens are assigned linear elastic material properties of shale rock. The ratio of elastic moduli of ceramic to walnut and shale rock to walnut is $80: 1$ and $4: 1$ respectively. Note that the analysis is large displacement large strain analysis which is activated by a keyword NLGEOM in ABAQUS, further discussion is provided in APPENDIX D. A discussion on metal plasticity is also provided in APPENDIX E.

The primary parameters of interest are stresses developed in the particles and the corresponding void spaces between them. In particular we are interested in observing the stress chains in the particles, the stress chains represent the path through which the load is transferred in the pack. The three models with $15 \%, 22 \%$ and $30 \%$ walnut particles by weight are compared by their load vs displacement response, the void fraction and total mixture weight.

\subsubsection{Results and Discussion}

The load vs displacement response for the three compositions of the walnutceramic mixture models is presented in Fig. 2.2. As mentioned previously the loading is displacement controlled and hence the load is measured as a reaction force at the reference node of the rigid element layer of platen (A). It is observed that the response stiffens with the increase in percent of hard particles, which is expected. Furthermore, the load vs displacement curve is observed to be nonlinear. This nonlinearity is due to the nonlinear inter-particle interaction and the nonlinear material response of the walnut 
as well as the ceramic particles. The curve has two distinct phases, the first one is observed in the lower load range where for small values of force significant displacement is observed, the response later stiffens in the second phase. The initial response is primarily due to particles undergoing rearrangement with little deformation. This stage of response could be significantly affected by the initial particle placement. For the current simulation the three mixtures have the same initial particle configuration and hence their response in the initial low load range closely matches. The second stage is of consolidation where the particles begin to carry significant loads and begin to undergo inelastic deformation. This stage of response is more dependent on the mixture composition and it is observed that a stiffer response is observed for the $15 \%$ walnut mixture compared to the $22 \%$ with the $30 \%$ walnut mixture being the least stiff.

Table 2.1 represents the force, void fraction and total particle mass (of the 60 particles) for the three walnut-ceramic mixtures at $0.75 \mathrm{~mm}$ platen displacement. Void fraction is computed using an image processing tool (ImageJ [62]) by measuring the number of pixels representing the deformed particles and comparing it with the pixels representing the entire domain (particles + voids) enclosed between all the platens. As stated previously force here is the reaction force at the reference node of the rigid element layer of platen (A). As the fraction of walnut particles increases, the mass of the pack mix decreases. For the same platen displacement the void fraction remains almost constant. Note that the reaction forces are reduced significantly as the weight percent of ceramic particles decreases (i.e. increasing walnut percent weight). For our problem we are primarily concerned with the load carrying capacity. $30 \%$ model looses $\sim 50 \%$ load carrying capacity compared to $15 \%$. Corresponding gain in weight reduction is much lesser. The void fraction is primarily dependent on platen displacement. The $30 \%$ model has approximately the same void fraction as $15 \%$ model but at half the load. 


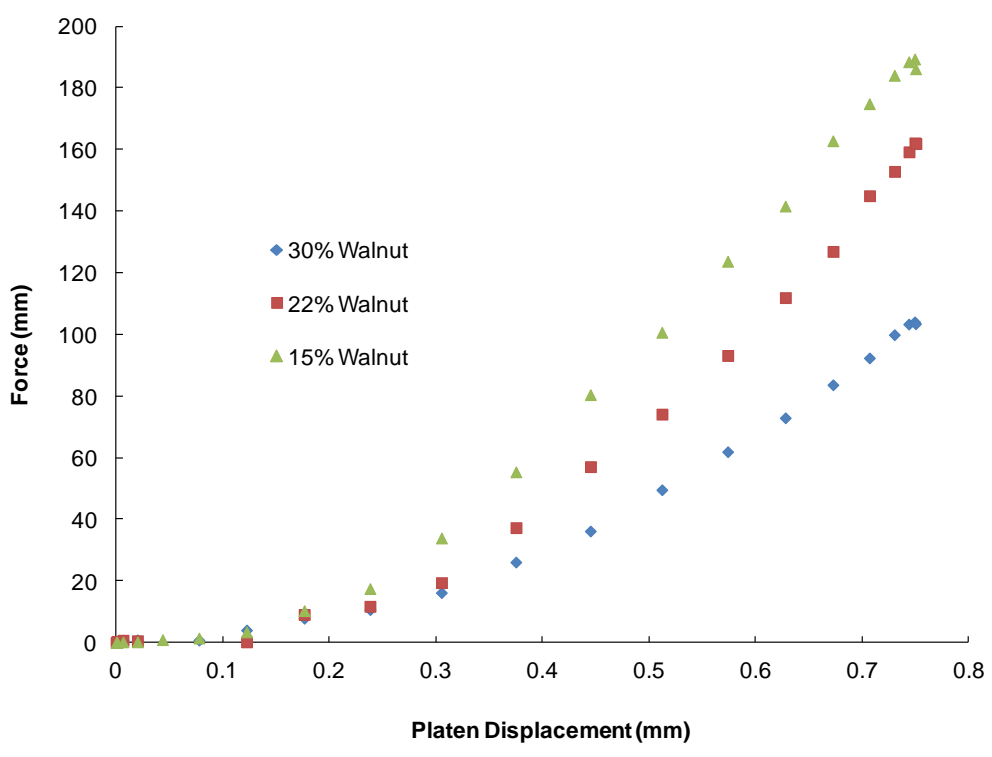

Fig. 2.2. The load vs displacement response of the three walnut-ceramic mixture models

Table. 2.1. Force, Void Fraction and total particle mass for the three walnut-ceramic mixtures at platen displacement of $0.75 \mathrm{~mm}$

\begin{tabular}{|c|c|c|c|}
\hline Model Type & Force (N) & Void Fraction & $\begin{array}{c}\text { Total Particle } \\
\text { Mass (Kg) }\end{array}$ \\
\hline I-15\% & 196 & 0.0726 & $2.5 \mathrm{e}-5$ \\
\hline II-22\% & 161 & 0.0710 & $2.3 \mathrm{e}-5$ \\
\hline III-30\% & 103 & 0.0875 & $2.1 \mathrm{e}-5$ \\
\hline
\end{tabular}

Fig. 2.3 and 2.4 show the von Mises stress contours for the $22 \%$ and $30 \%$ by weight walnut models. The particles which show higher stress are the ceramic particles. The force applied from the top platen is transferred to the bottom platen via the ceramic particles. The path of this force transfer is termed as force/stress chain. Comparing Fig. 2.3 and 2.4 we can observe how the stress chains deviate based on composition. This 
behavior of granular materials is at variance to that observed in homogeneous solids which show uniform stress on undergoing uniaxial compression. Correspondingly comparing the equivalent plastic strain contours for the $22 \%$ and $30 \%$ walnut mixtures, Fig. 2.5 and 2.6 it is observed that the walnut particles undergo a much higher deformation compared to the ceramic particles. This also explains the reduction in the stiffness of the load vs displacement response for the models with higher percent of softer particles.

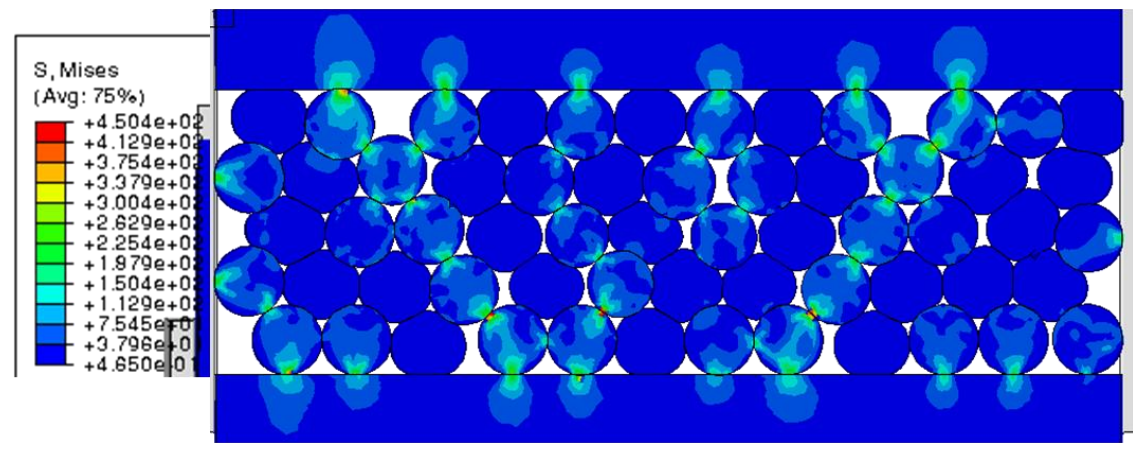

Fig. 2.3. Von Mises stress contour for $22 \%$ walnut model at $0.75 \mathrm{~mm}$ platen displacement

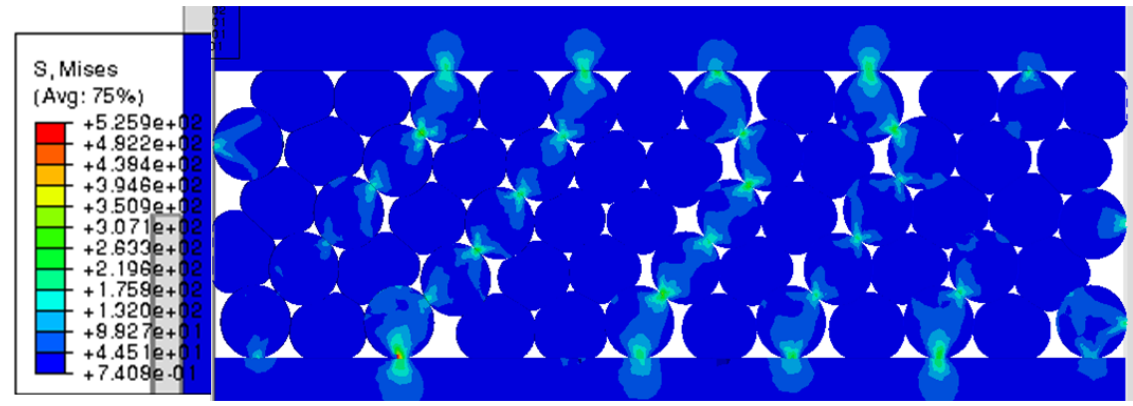

Fig. 2.4. Von Mises stress contour for $30 \%$ walnut model at $0.75 \mathrm{~mm}$ platen displacement 


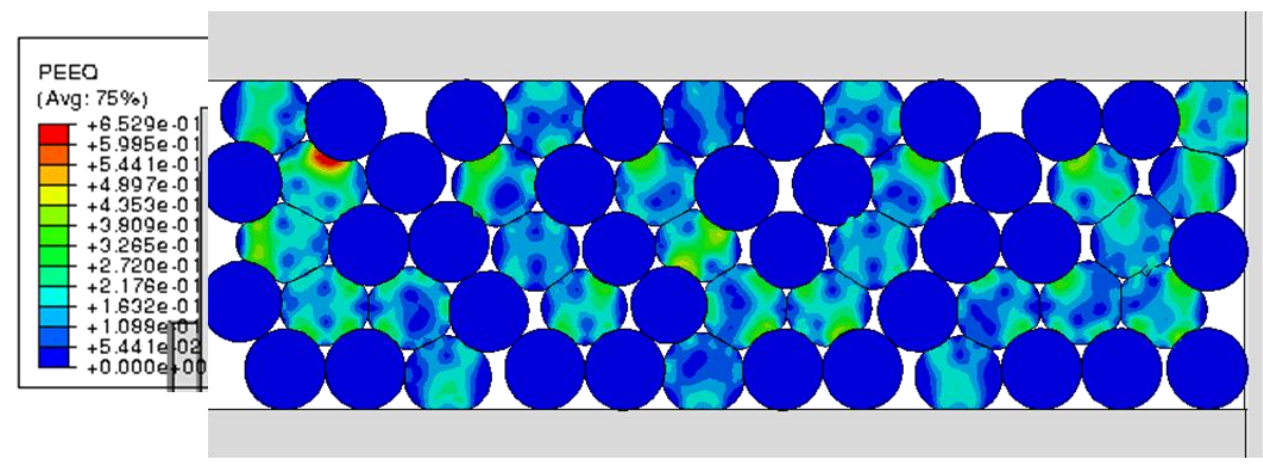

Fig. 2.5. Equivalent plastic strain (PEEQ) contour for $22 \%$ walnut model at $0.75 \mathrm{~mm}$ platen displacement

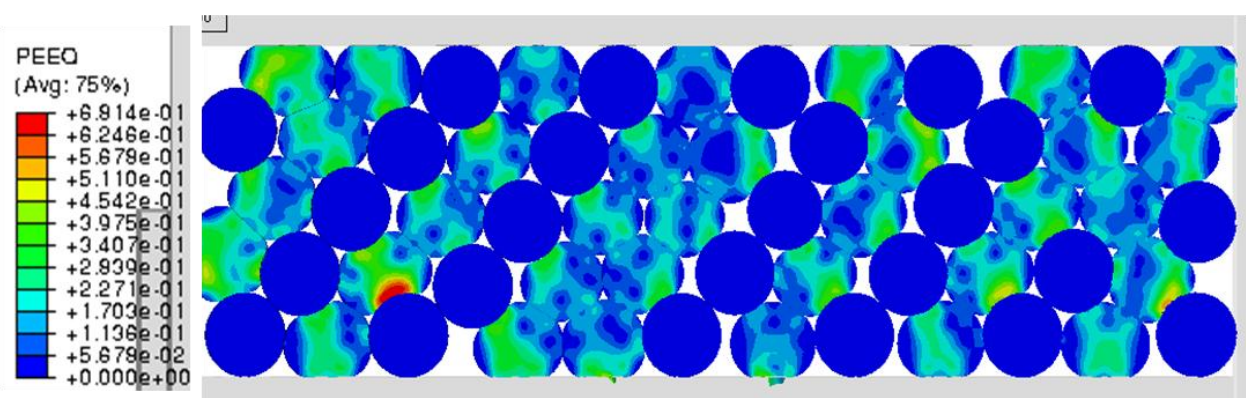

Fig. 2.6. Equivalent plastic strain (PEEQ) contour for $30 \%$ walnut model at $0.75 \mathrm{~mm}$ platen displacement

\subsection{Flowback Models}

As is discussed previously in section 1.1 flowback is the outflow of proppants from the hydraulic fracture along with the flow of oil/gas. This load case is modeled in the vicinity of the well bore. The proppant flowback is also termed as proppant back production and is detrimental to the safety and life of the well and production equipments. There are two primary loads acting on the proppants in this case, one is the 
compressive load coming from the rock surfaces and second is a transverse force i.e. perpendicular to the compressive force which acts on the particles due to fluid flow and pressure gradient (also known as drawdown) along the length of the fracture. The transverse forces (drag forces) tend to push the proppants in the flow direction dislodges them and impacts the pack stability.

A detailed discussion on determining the drag forces is presented in Asgian et al [14]. To summarize, drag forces have two components, pressure and shear. The pressure component is due to the pressure gradient existing along the fracture length. This component is given by the equation $F_{P}=(4 / 3) \pi r^{3}(d P / d x)$. Here $F_{P}$ is the pressure component of the drag force, $\mathrm{r}$ is the proppant radius and $\mathrm{dP} / \mathrm{dx}$ is the pressure gradient in the fracture along the flow direction. For the shear component, an analytic solution for laminar flow around isolated spherical particles is applied to the flow in the fracture, this gives the shear force as $F_{S}=6 \pi v_{\infty} \mu r$. Here $v_{\infty}$ is the average pore velocity and is calculated as Darcy velocity divided by the effective porosity, $\mu$ is the dynamic viscosity. Further this shear force is written in the form where $\mathrm{v}_{\infty}$ is replaced with the pressure gradient, effective porosity of the medium $\varphi$ and permeability, $F_{S}=(6 \pi / \varphi) \mathrm{k}(\mathrm{dP} / \mathrm{dx}) \mathrm{r}$. In the next step the lower bound and the upper bound for the shear forces are determined by comparing it to the pressure drag and then considering the overall force balance on the fluid within the pore space. From this analysis the lower value of the shear component is zero while the upper bound is always lesser than the pressure component. Finally as a conservative estimate the overall drag force on the proppant particles is given as $\mathrm{F}_{(\mathrm{P}+\mathrm{S})}=$ $(8 / 3) \pi r^{3}(\mathrm{dP} / \mathrm{dx})$. Note that the upper bound for the shear force is obtained by assuming uniform size particles and effective porosity $\varphi$ of the medium to be less than $50 \%$.

The current simulation models (Fig. 2.7) accommodate 240 particles, each $1 \mathrm{~mm}$ in diameter, in the fracture width ( $\mathrm{fw}$ in Fig. 2.1) of $4.5 \mathrm{~mm}$. The fracture length ( $\mathrm{fl}$ in Fig. 2.1) is $52 \mathrm{~mm}$ and accommodates approximately 50 particles. The load is applied over three steps i) to avoid spurious proppant production from the unconstrained end which represents well bore opening during the compression process the compressive load is applied in the same manner as the confined compression load case, ii) after the 
pack is under compression one of the rigid side platens is removed, the unconstrained end then represents the well bore opening and a few particles at this end then become free and start coming out of the fracture finally iii) the transverse load representing fluid drag force is applied on the proppants. The three steps in sequence are represented in Fig. 2.8 (a) (b) and (c). The loading is incremental and quasi-static, and the confined compression stage is force controlled. The concentrated force is applied at the reference node of the rigid top layer of the rock platen. For the present simulation the confined compression load is limited to $250 \mathrm{~N}$, equivalent to $48 \mathrm{MPa}$ pressure. The transverse drag force is applied as uniformly distributed load over the particles; it is modeled as a concentrated force on every node of the particles. In this model the force is equivalent to a pressure gradient of $1.686 \mathrm{MPa} / \mathrm{m}(75 \mathrm{psi} / \mathrm{ft})$. The flowback resistance of a proppant pack is studied by observing the number and distance from the unconstrained end over which particles fall out of the pack and the formation of a stable arch. Two different packs are considered; first one has $15 \%$ walnut and $85 \%$ ceramic particles and the second one consists of $100 \%$ ceramic particles. Two models with coefficient of friction $\mu=0$ and 0.3 are considered to study the influence of inter-particle friction. The effect of closure stress (compressive load) on flowback is considered by comparing particle response at $50 \mathrm{MPa}$ and $100 \mathrm{MPa}$ pressure for both packs without inter-particle friction. The influence of rock is addressed by treating shale rock as linear elastic as well as elastic-plastic material.

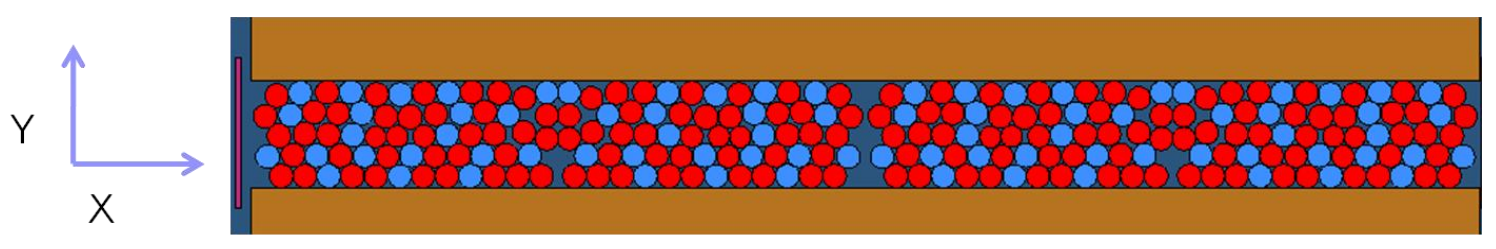

Fig. 2.7. Flowback model with $15 \%$ walnut particles (blue) by weight 


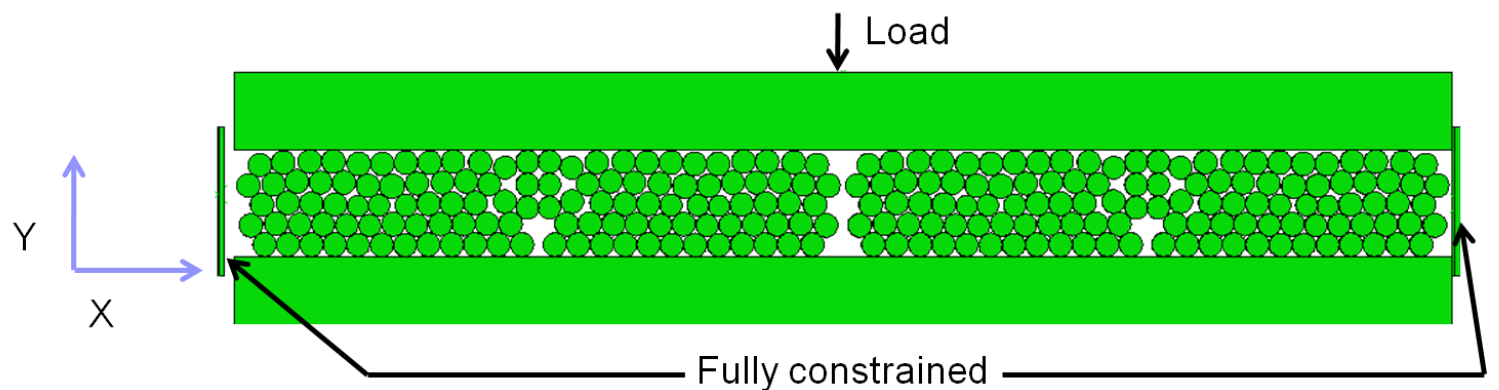

(a)

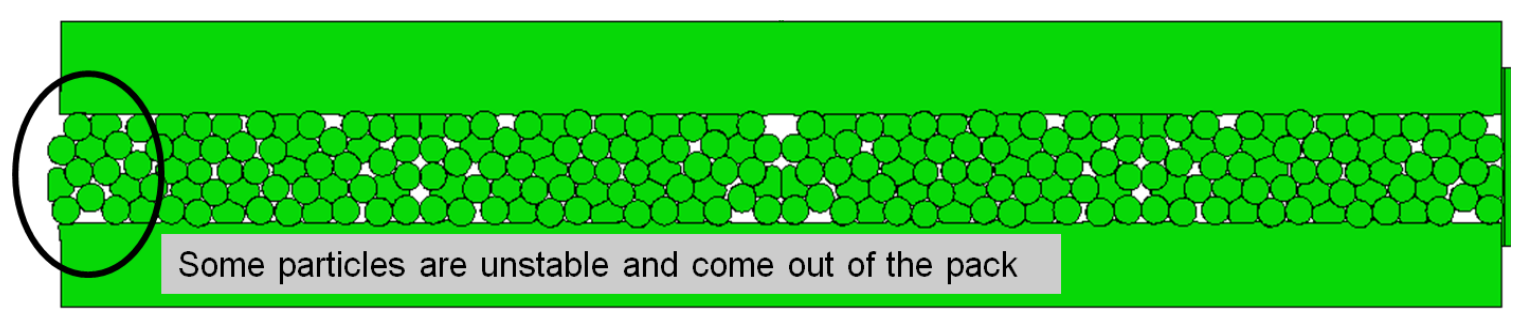

(b)

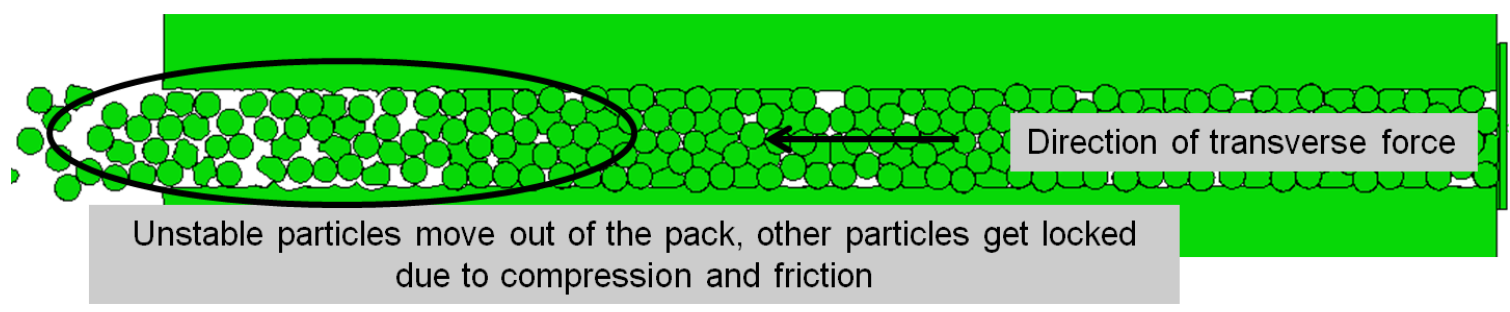

(c)

Fig. 2.8. (a) First step of flowback loading, confined compression, (b) removal of side platen and (c) application of transverse load resulting in unstable particles moving out of the pack 


\subsubsection{Results and Discussion}

The results show that friction, nonlinear material behavior, and high closure stresses significantly resist particle flowback. The transverse nodal velocity contours for $15 \%$ walnut mixture subjected to $48 \mathrm{MPa}$ closure stress with $\mu=0.3$ for elastic (hard) and elastic-plastic (soft) rock, are presented in Fig. 2.9a and 2.9b respectively. A stable arch which significantly limits proppant flowback is observed (highlighted) in case of a plastically deformed rock with ceramic particles embedded in it, Fig. 2.9b. The transverse motion of the particles is resisted by the inter-particle and particle-rock friction, particle interlocking and particle embedment. In case of elastic rocks, very little particle embedment takes place leading to a higher flowback. A similar result is observed for the case with $\mu=0$, presented in Fig. 2.10a and 2.10b respectively for elastic and elastic-plastic rock. Again comparing Fig. 2.9a with 2.10a (elastic rock) and Fig. $2.9 \mathrm{~b}$ with $2.10 \mathrm{~b}$ (elastic-plastic rock) the influence of coefficient of friction on flowback resistance is evident. For the case with elastic rock, for $\mu=0$ condition the pack is observed to have collapsed completely while for $\mu=0.3$ even though we don't

clearly observe a stable arch the pack is relatively stable due to interparticle friction. For the case with elastic-plastic rock it is observed that a stable arch is formed at a very short distance from the unconstrained end for $\mu=0.3$. This is attributed to the combined action of particle interlocking, inter-particle friction and particle embedment in the deformable rock. For the same case with $\mu=0$ we don't clearly observe a stable arch and particles seem to be freely moving over a significantly longer distance from the unconstrained end. Note that only particle interlocking and embedment occur for the frictionless condition. 


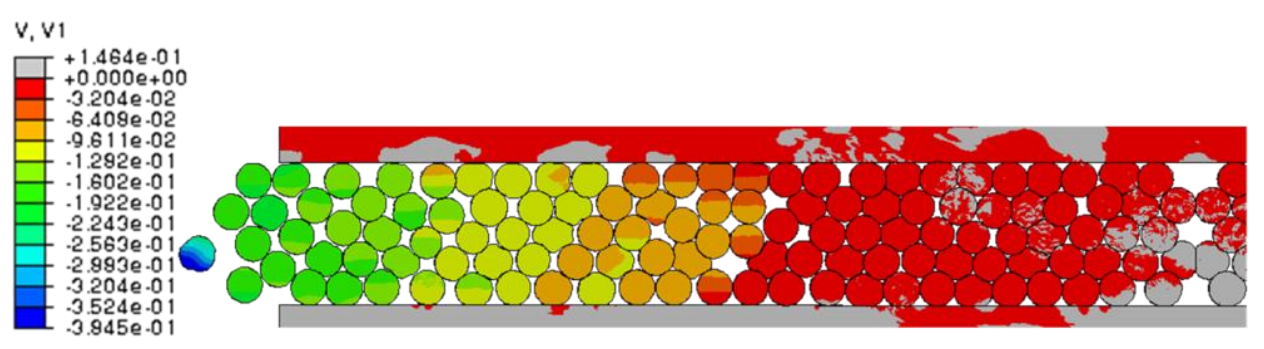

(a)

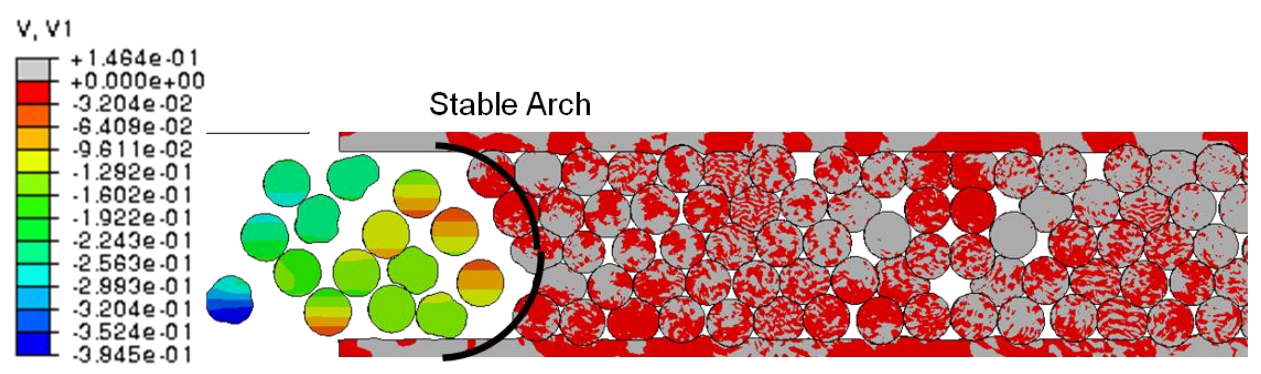

(b)

Fig. 2.9. Transverse nodal velocities: $15 \%$ walnut model at $48 \mathrm{MPa}$ pressure for $\mu=0.3$

(a) linear elastic rock and (b) elastic-plastic rock

Comparing Fig. 2.10b (48 MPa pressure) and Fig. 2.11 (96 MPa pressure) it can be concluded that a higher compressive load aids in preventing flowback and results in a stable arch, this is primarily due to deeper particle embedment and more particle interlocking. On the other hand at high pressures the pack is highly compressed with low porosity and correspondingly results in a loss of fluid flow rate. 

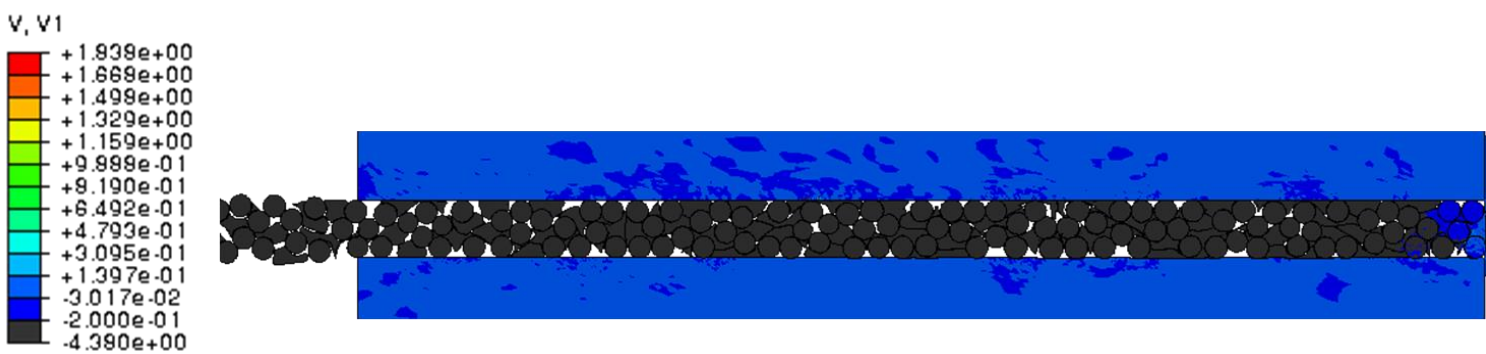

(a)
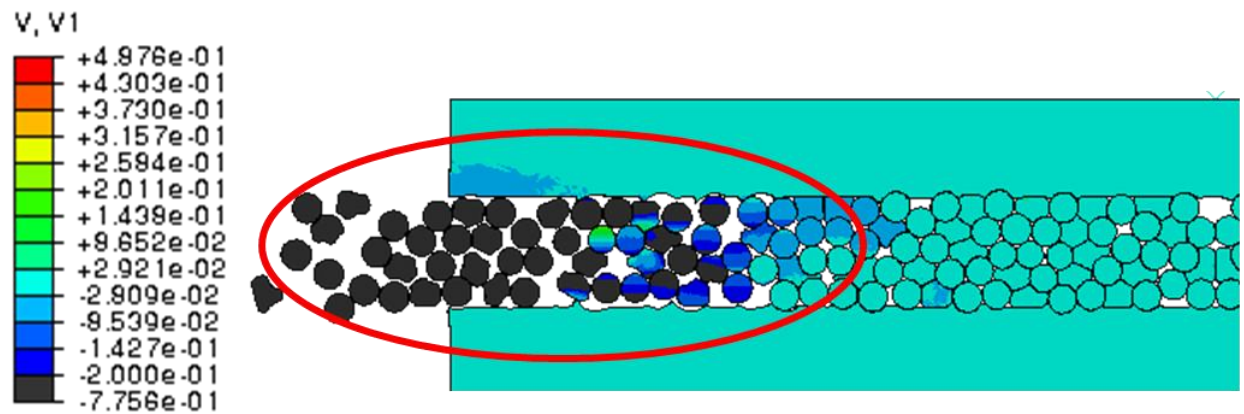

(b)

Fig. 2.10. Transverse nodal velocities: $15 \%$ walnut model at $48 \mathrm{MPa}$ pressure for $\mu=0$,

(a) linear elastic rock and (b) elastic-plastic rock
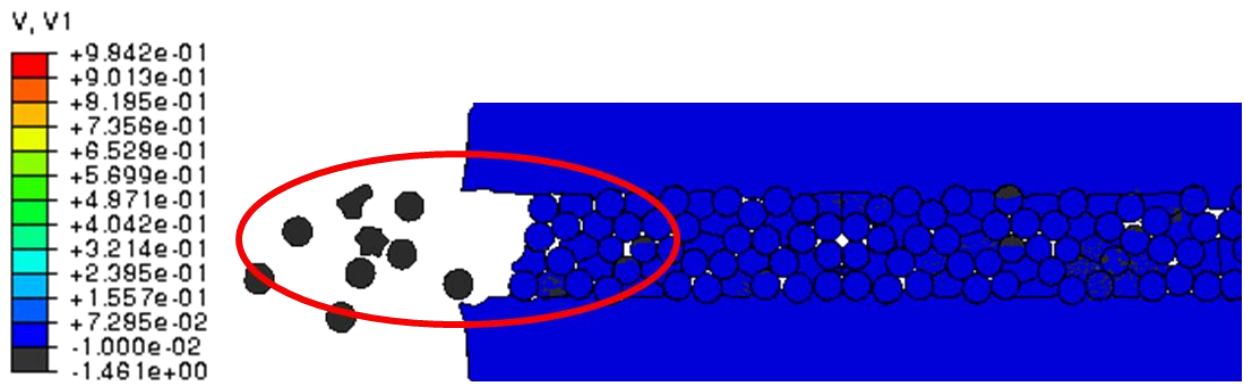

Fig. 2.11. Transverse nodal velocities: $15 \%$ walnut model at $96 \mathrm{MPa}$ pressure for $\mu=0$ and elastic-plastic rock response 
Fig. 2.12 shows the transverse nodal velocity contours for the $100 \%$ ceramic particle pack with elastic-plastic rock at $48 \mathrm{MPa}$ closure stress, $\mu=0.3$. The stable arch is highlighted in Fig. 2.12. Comparing Fig. $2.9 \mathrm{~b}$ and 2.12 it is observed that the distance from the unsupported end at which a stable arch is formed is shorter in case of the walnut-ceramic mixture. This is due to larger deformation experienced in walnut particles that leads to greater particle interlocking. It is also noted that the pore space is reduced for the $15 \%$ walnut mixture compared to the $100 \%$ ceramic particle pack which can result in a loss of flow rate. A similar result is observed for the case with $\mu=0$ at an applied pressure of $96 \mathrm{MPa}$ in Fig. 2.11 and 2.13. We can observe a stable arch formed at a shorter distance from the unconstrained end for the $15 \%$ walnut-ceramic mixture compared to the $100 \%$ ceramic pack. We also observe that the $15 \%$ walnut-ceramic mixture is compressed to a much higher extent resulting in significant loss of porosity compared to the $100 \%$ ceramic pack which shows much higher porosity.
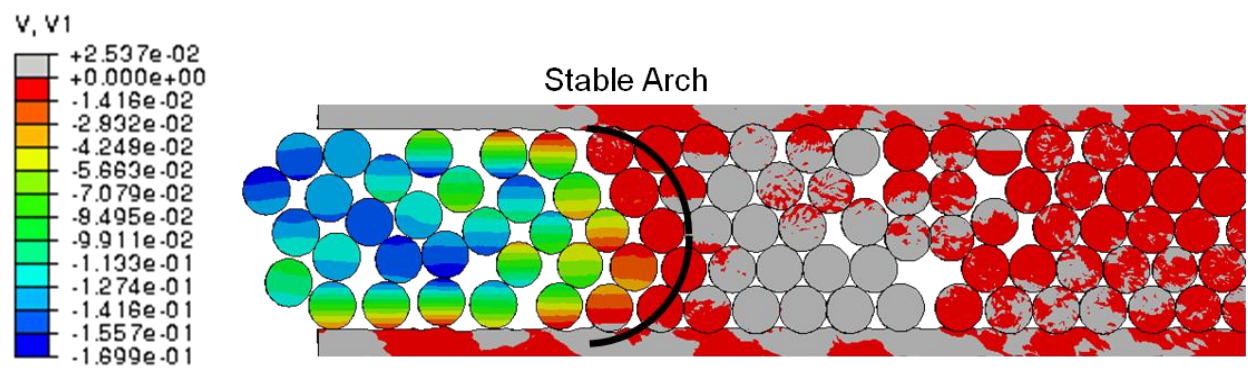

Fig. 2.12. Transverse nodal velocities: $100 \%$ ceramic pack at $48 \mathrm{MPa}$ pressure for $\mu=$ 0.3 with elastic-plastic rock 


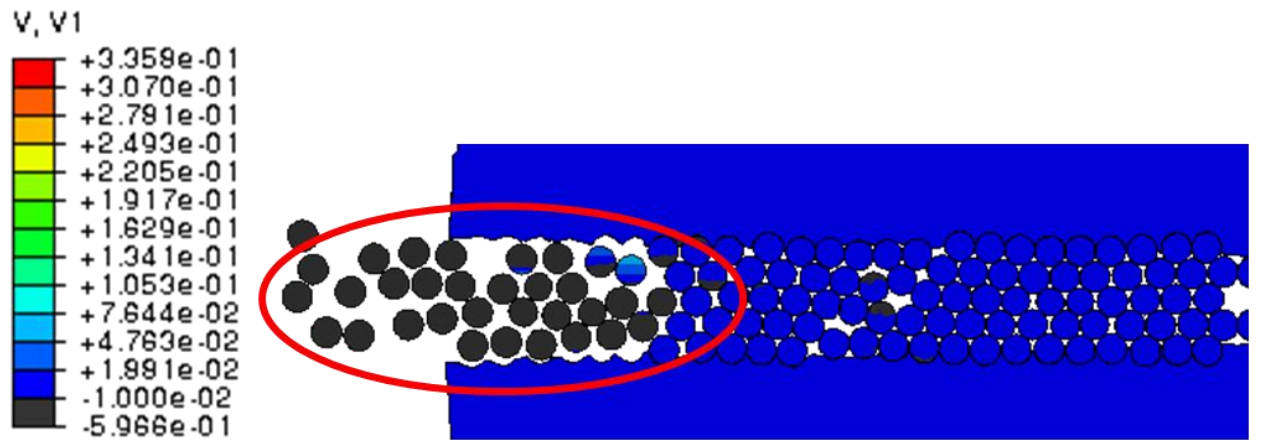

Fig. 2.13. Transverse nodal velocities: $100 \%$ ceramic pack at $96 \mathrm{MPa}$ pressure for $\mu=0$ and elastic-plastic rock

These results are consistent with the observations reported in literature which state that i) Formation of a stable arch is necessary to obtain a stable pack [14], ii) Formation of a stable pack is easier with softer rock platens which permits deeper particle embedment [15] iii) Presence of softer deformable particles in the pack increases flowback resistance, but will result in loss of porosity and subsequent loss of fluid flow rate [49-51]. 


\section{ANALYSIS OF POLYDISPERSE PARTICLES}

This chapter introduces polydisperse particle models and results. Two categories of particle mixtures are considered; Type-A mixture of ceramic and walnut, and Type-B mixture of ceramic and pure aluminum (Al). Particles have non-uniform sizes and shapes. The combined finite/discrete element technique in the commercial software ABAQUS v 6.8.3 with explicit time integration is exercised. The section begins by elaborating on the procedure to obtain a polydisperse particle pack. This is followed by models of different particle mixtures subjected to confined compression load case. Influence of initial particle arrangement on load vs displacement is studied. The change in pack porosity with pack composition, particle shape and inter-particle friction are also studied. Two cases of constitutive ceramic behavior are studied. In the first case, ceramic particles are represented with concrete damage plasticity as discussed in section 2. In the second case, they are assigned to follow the concrete brittle cracking model and particle fragmentation behavior is implemented through element deletion technique.

\subsection{Initial Particle Placement}

The first step to modeling a granular pack is to obtain an initial randomly placed particle model with size and shape distribution. Further, prior to the application of compressive load on a granular pack, it needs to be ensured that the pack is stable. Before introducing the modeling approach employed, a brief literature survey of common techniques to generate the initial particle arrangement is presented.

\subsubsection{Literature Survey on Pack Configuration}

In practice DEM is used to extensively study the detailed microstructure of particle packing. In this approach an initial random particle arrangement is prepared and then allowed to settle while the particles are subjected to excitations, this gives us a segregated initial packing arrangement. Also techniques like computer tomography 
(CT) and nuclear magnetic resonance (NMR) are used to visualize particle packing [25]. The porous microstructure captured from micro-CT can be used to generate three dimensional mesh of the microstructure. This method has been employed to model porous media like carbon foam [63]. One other approach involves using techniques like Monte-Carlo method to generate two or three dimensional particle segregation, this work on the principle of minimization of potential energy of the pack [64]. This apart ballistic deposition algorithms can be employed to generate an initial particle packing [65, 66]. The combined FEM/DEM approach along with the ballistic deposition algorithms have been used to model particle segregation $[67,68]$. In some cases the random number generation algorithm in software like MATLAB are utilized to generate an initial random loose packing with a pack density of less than $50 \%$. The particles are then modeled in either explicit FEM or DEM and then allowed to fall freely under gravity. This free fall results in generation of a packing arrangement which is close to that obtained with a ballistic deposition algorithm. The randomization algorithm can also give us random distribution of hard and soft particles in a particle mixture. During this simulation it needs to be ensured that particles don't undergo significant plastic deformation $[42,43]$.

\subsubsection{Generating a Polydisperse Pack}

In our research, the random number generation function in MATLAB is invoked to generate an initial random particle distribution in a rectangular domain. This function is also used to assign random distribution of hard and soft particles. Polydispersity of particles is incorporated by assigning random numbers for particle radii. These follow a Gaussian distribution and are related with the random numbers which indicate particle center. By limiting the center distance between any two particle centers to be greater than the sum of their individual radii particle overlap is prevented. The MATLAB script is attached in APPENDIX C. The data for particle radii and center co-ordinates is incorporated as a macro (Python script) in ABAQUS CAE v 6.8.3 to generate a solid 
model of polydisperse particle distribution. Fig. 3.1 shows two such initial low density configurations of a mixture of hard and soft particles. The large particle diameters range from $\sim 0.8-1.2 \mathrm{~mm}$, while the small particles range from $0.4-0.6 \mathrm{~mm}$, in this particular case the soft particles constitute $15 \%$ of the total number of particles. Note that the particle arrangement has a very loose packing and the void fraction is more than 0.5 . This is not a stable pack and cannot be subjected to compressive loading directly.

The finite element modeling of the loose configuration is carried out in the preprocessing software Altair Hypermesh V9 (Altair Engineering Inc, MI). Particles are enclosed within a rectangular domain and are subjected to gravitational loading allowing particles to fall freely in ABAQUS explicit 6.8.3, Fig. 3.2a. During this step particles are treated as linear elastic with a high value of elastic modulus to prevent deformation due to impact. Three dimensional continuum elements, C3D8R, with eight nodes and reduced integration are used to model the particles and the rock platens. The spherical particles are represented as cylinders for simplicity. Plane strain conditions are enforced along the cylinder axis. The largest particles are composed of 1200 elements while the smallest particles are described with 150 elements. Fig. 3.2a shows a representative model with 150 particles. The particle configuration at the end of free fall (Fig. 3.2b) is imported into a new analysis employing the *IMPORT keyword and the particles are then assigned appropriate linear or nonlinear material properties. This approach removes the need to monitor the plastic strains developed during free fall which is required in $[42,43]$. The imported configuration is then subjected to compressive loading, via the top rock platen. For confined compression load case the boundary conditions are similar to those described in section 2.2. For the above mentioned representative model with 150 particles the compressed pack is shown in Fig. $3.2 \mathrm{c}$. 


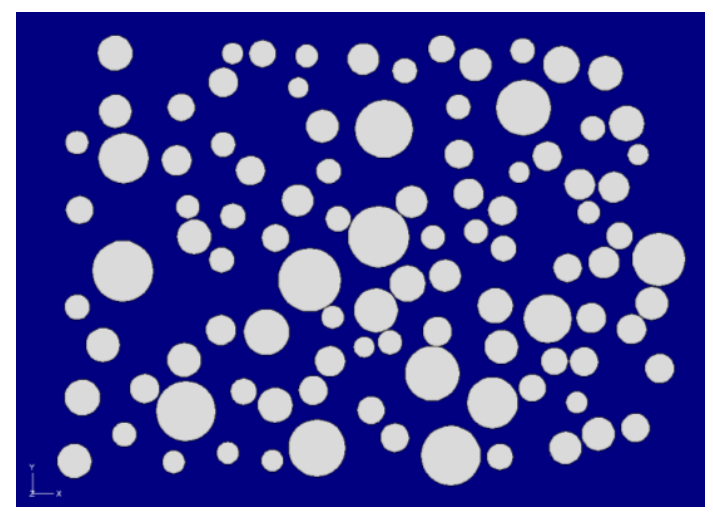

(a)

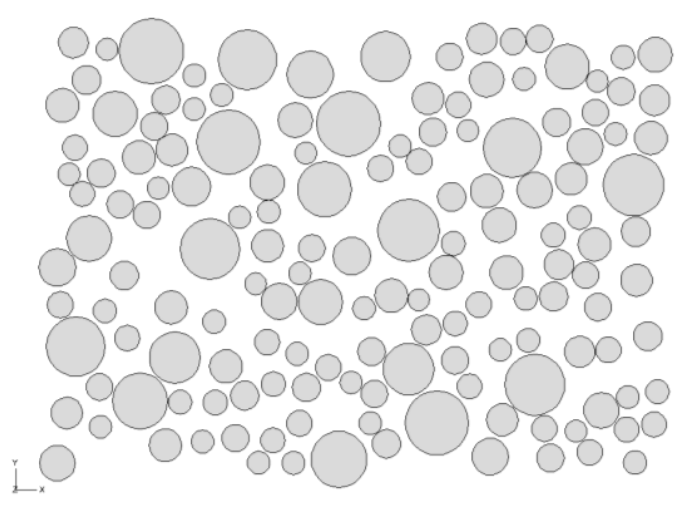

(b)

Fig. 3.1. Models with 100 particles (a) and 150 particles (b) showing the initial low density particle configuration

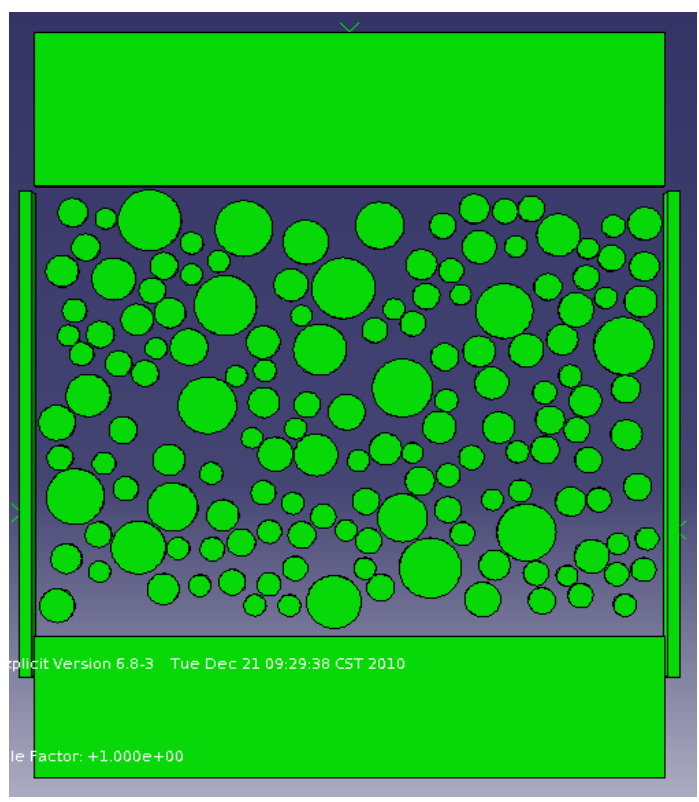

(a)

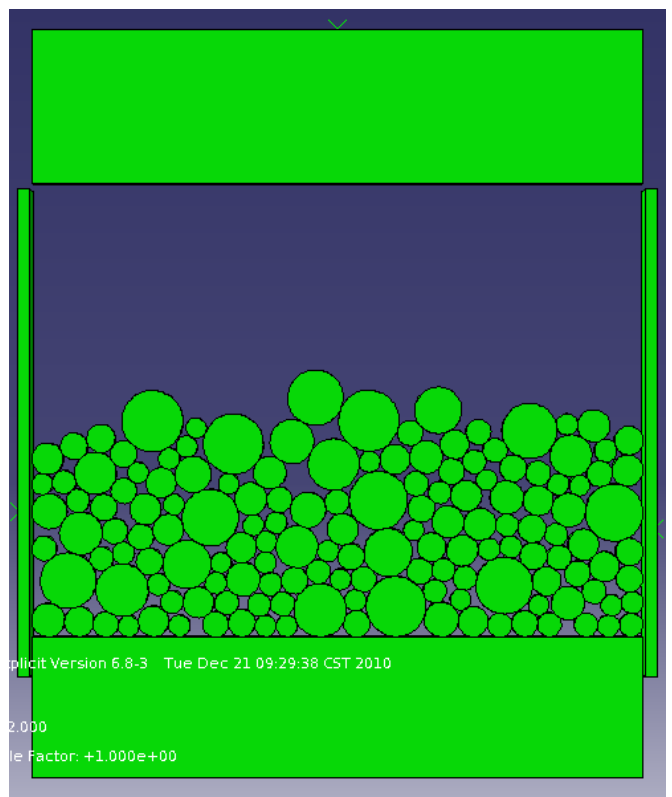

(b)

Fig. 3.2. (a) Loose particle configuration with a rectangular domain (b) Particle configuration at the end of free fall (c) Imported particles in new analysis at the end of compressive loading 


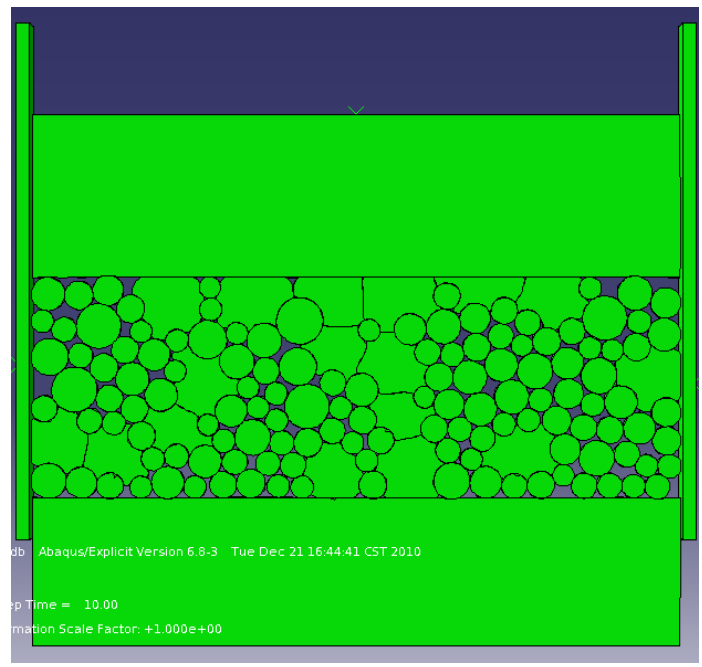

(c)

Fig. 3.2. Continued

\subsection{Polydisperse Pack Response Studies}

Following the procedure described above, further computational models are developed to study the influence of mixture composition, soft particle type and interparticle friction on the compression response of a granular pack. Only the confined compression load case is documented here. The first class of models described here is simulated by introducing damage into the ceramic particles without allowing particle fragmentation

\subsubsection{Model Description}

The boundary conditions for confined compression case remain same as discussed in section 2.2. For simplicity and reducing the computational cost 2D simulation is considered. The load case is simulated by placing the pack between the 
two rock platens and two side platens as shown, in Fig. 2.1 section 2.2. The distance between the two rigid platens is limited to $26 \mathrm{~mm}$. Compressive force (max 150N) is applied at the reference node of the rigid element layer equivalent to a pressure of 57 $\mathrm{MPa}$. The analysis is quasi-static and mass scaling is employed to speed up the calculations. Inertia effects are monitored to ensure their effects on the results are negligible. Prior to the application of compressive load the randomly arranged particles in the sparse packing are subjected to gravitational loading and allowed to undergo free fall. At the end of free fall for each of the different mixtures considered depending on particle settlement the fracture width $\left(f_{w}\right.$ - Fig. 2.1 section 2.2$)$ varies between $4-6 \mathrm{~mm}$.

Three dimensional continuum elements, C3D8R, with eight nodes and reduced integration are used to model the particles and the rock platens. The model consists of 400 particles with 113,688 elements and 247,686 nodes. Two different types of particle mixtures are studied, Type-A consists of oddly shaped but rounded walnut and spherical ceramic particles. For simplicity the oddly shaped walnut shell particles are simulated as cylinders in 2D as are the ceramic particles. The randomly distributed particles for Type-A mixture of $10 \%$ walnut by weight prior to free fall are observed in Fig. 3.3a. The configuration of the particles at the end of free fall is shown in Fig. 3.3b. Type-B is a combination of prismatic $99 \%$ pure aluminum $(A l)$ and spherical ceramic particles. Fig. 3.4 shows the Type-B mixture ( $25 \%$ pure Al by weight) at the end of free fall. To assess the impact of softer material property on the pack response a harder alloy of $\mathrm{Al}$ is also considered, the response due to hard and soft Al particles are compared. The particle elastic material property, shape and size range are summarized in Table - 3.1. Walnut and $A l$ as softer particles are treated as elastic-plastic materials. Walnut data is obtained from single particle compression tests on walnut shell specimens $[59,60]$ this data is same as used for the uniform size particle models in section 2.2. The yield strength of $A l$-pure and a harder $A l$-alloy is taken as $50 \mathrm{MPa}$, and $270 \mathrm{MPa}$ respectively [69]. Ceramic is represented as a quasi-brittle material and simulated with the concrete damage plasticity model described in [23, 61], discussed in APPENDIX B. It remains linear-elastic until tensile stresses reach its bending strength (here $180 \mathrm{MPa}$ ). 


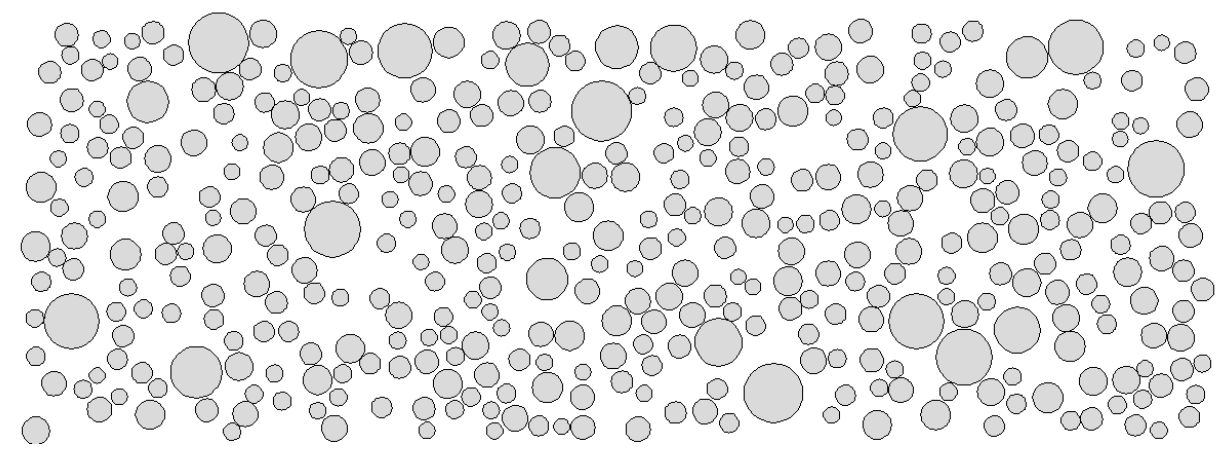

(a)

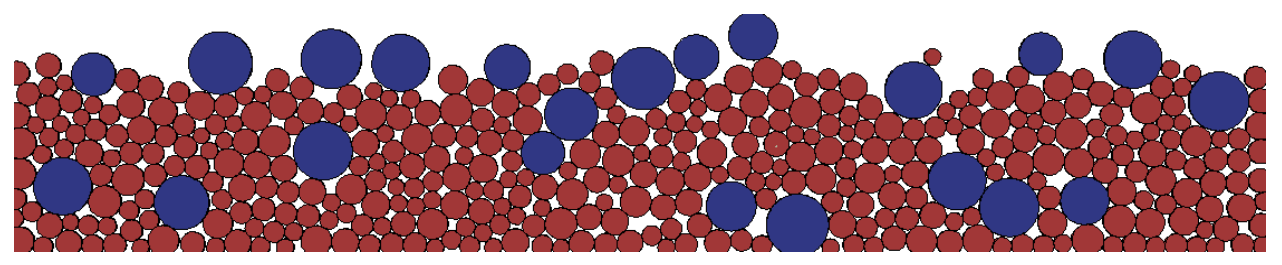

(b)

Fig. 3.3. Type-A mixture, 10\% walnut shell by weight: (a) Randomly generated polydisperse particles (b) particles at the end of free fall

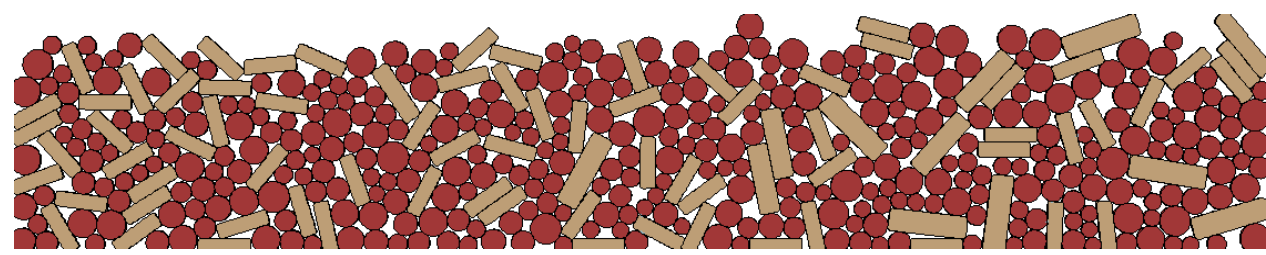

Fig. 3.4. Particle configuration after free fall for $25 \%$ pure Al, Type-B mixture 
Table. 3.1. Material properties and particle description

\begin{tabular}{|c|c|c|c|c|c|}
\hline Material & $\begin{array}{c}\text { Elastic } \\
\text { modulus } \\
(\mathbf{G P a})\end{array}$ & $\begin{array}{c}\text { Poisson's } \\
\text { ratio }\end{array}$ & $\begin{array}{c}\text { Specific } \\
\text { gravity }\end{array}$ & $\begin{array}{c}\text { Section } \\
\text { Shape }\end{array}$ & $\begin{array}{c}\text { Particle size } \\
\text { range (mm) }\end{array}$ \\
\hline Ceramic & 259 & 0.25 & 3.6 & Circle & $0.35-0.55$ \\
Walnut shell & 3.7 & 0.3 & 1.25 & Circle & $0.8-1.2$ \\
Pure Al & 70 & 0.3 & 2.7 & Prismatic & $(1-2) \mathrm{X}(0.35-0.7)$ \\
Al alloy & 70 & 0.3 & 2.7 & Prismatic & $(1-2) \mathrm{X}(0.35-0.7)$ \\
\hline Shale Rock & 12 & 0.22 & 2.5 & - & - \\
\hline
\end{tabular}

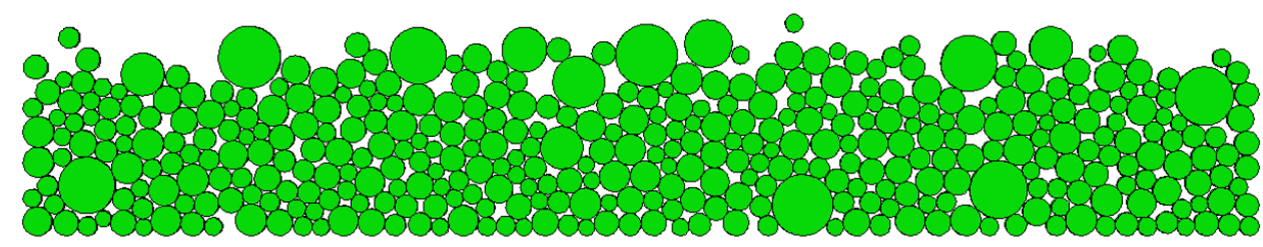

Fig. 3.5. Particle configuration after free fall for $6 \%$ walnut shell, Type-A mixture

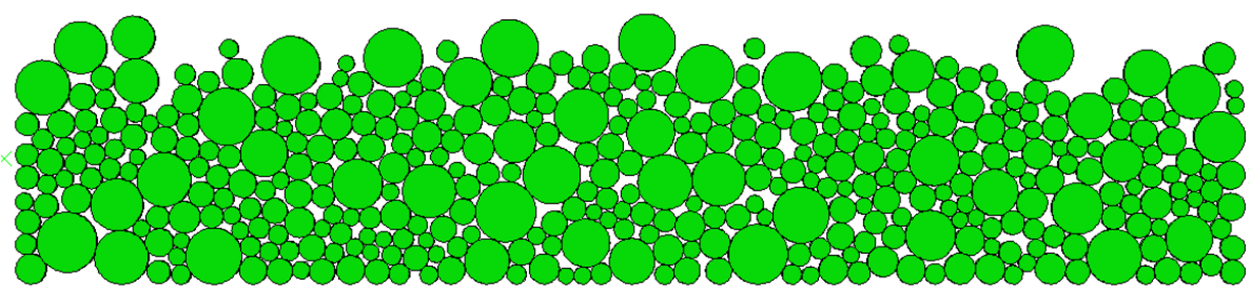

Fig. 3.6. Particle configuration after free fall for $18 \%$ walnut shell, Type-A mixture 


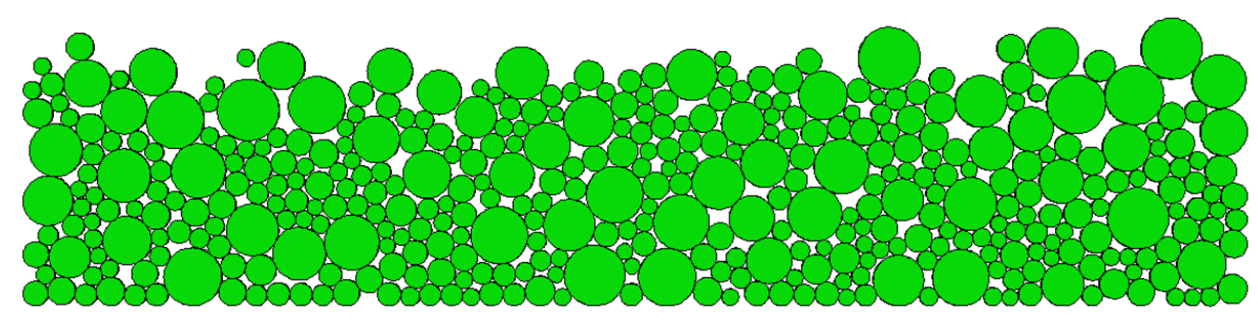

Fig. 3.7. Particle configuration after free fall for $25 \%$ walnut shell, Type-A mixture

Table. 3.2. Models A-B in simulations

\begin{tabular}{|c|c|c|c|}
\hline $\begin{array}{c}\text { Model } \\
\text { Type }\end{array}$ & Mixture & $\begin{array}{c}\text { Loading } \\
\text { condition }\end{array}$ & $\begin{array}{c}\text { \% Soft } \\
\text { particles added }\end{array}$ \\
\hline A & Walnut, Ceramic & $\begin{array}{c}\text { Confined } \\
\text { compression } \\
\text { Confined }\end{array}$ & $6,10,18,25$ \\
B & Al, Ceramic & $\begin{array}{c}\text { compression } \\
\text { com }\end{array}$ \\
\hline
\end{tabular}

The pack response is characterized in terms of pressure vs displacement response and the change in pack porosity with pressure. Pressure is represented as the ratio of the applied force to the platen area while displacement is non-dimensionalized by dividing it with the pack thickness at the end of free fall. To study the effect of mixture composition on the pack response, four different Type-A mixtures are considered. The configuration at the end of free fall for the four mixtures is presented in Fig 3.3b, 3.5, 3.6 and 3.7, note that the larger particles are walnut while ceramic are the smaller ones. The influence of particle material and inter-particle friction is illustrated in the corresponding von Mises stress contours of Type-A and B mixtures each with 25\% soft particles by weight and two different coefficients of friction $(\mu) 0.3$ and 0.03 . The different models are summarized in Table. 3.2. 
Another FEM model was developed for the 25\% walnut Type-A mixture with 850 particles. This model is developed to study the influence of pack thickness and number of particles on the response of the computational model. This model consists of 298767 C3D8R three dimensional continuum reduced integration elements and 644442 nodes. The boundary conditions and particle placement procedures are same as those employed for the 400 particles models discussed previously. For this model the rectangular domain has $36 \mathrm{~mm}$ distance between the two vertical rigid confining platens and the pack thickness at the end of particle settlement is $11 \mathrm{~mm}$, compared to $26 \mathrm{~mm}$ and $6.2 \mathrm{~mm}$ respectively for the 400 particle model. The particle configuration at the end of free fall for this model is presented in Fig. 3.8. The pressure vs non-dimensional displacement response of the 850 particle model is compared to the 400 particle $25 \%$ Type-A mixture.

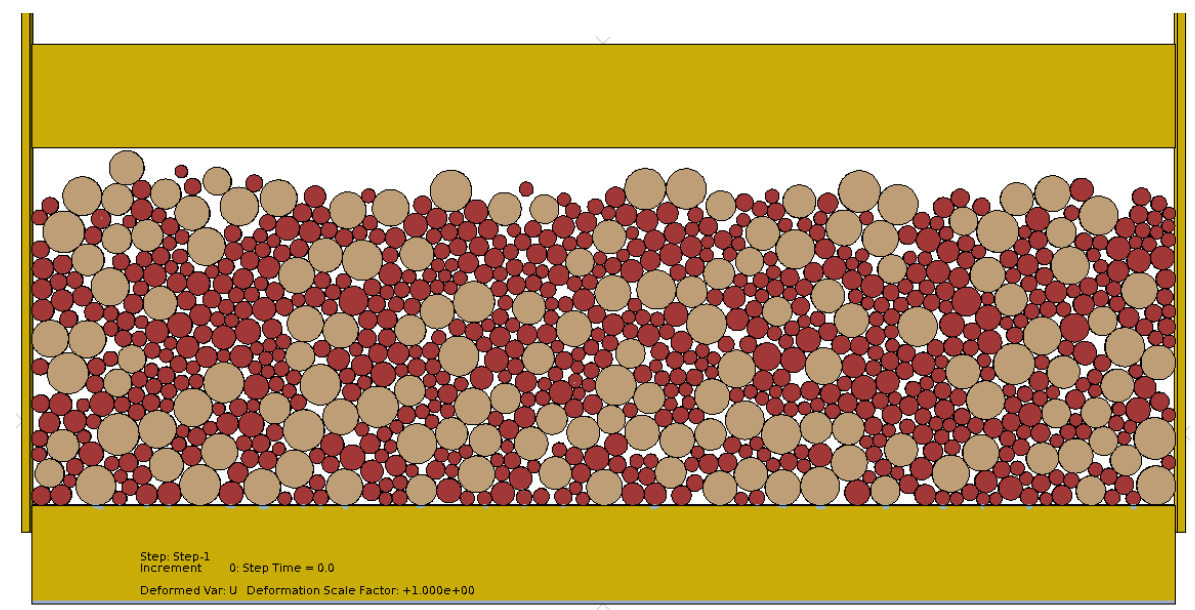

Fig. 3.8. Particle configuration at the end of free fall for the $25 \%$ by weight walnut particles mixture (light colored - walnut, dark shade - ceramic), 850 particles pack thickness $11 \mathrm{~mm}$, width $36 \mathrm{~mm}$. 


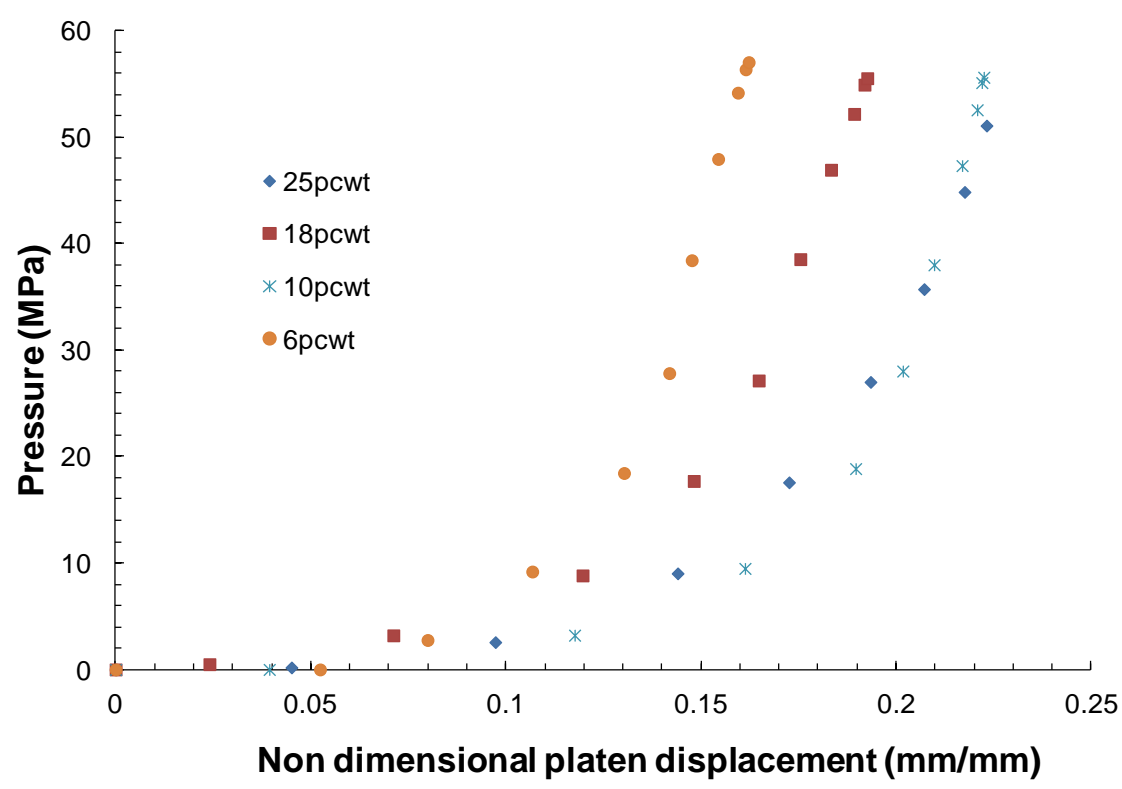

Fig. 3.9. Type-A mixtures, $\mu=0.3$, Pressure vs displacement

\subsubsection{Results and Discussion}

Effect of Percent Weight of Soft Particles

The pressure vs non-dimensional displacement response for the four Type-A mixtures, $\mu=0.3$ are presented in Fig. 3.9. The nonlinear response is typical for granular materials with an initial particle rearrangement followed by densification and particle deformation; this is similar to the response observed for uniform sized particles in section 2.2.1. Notice that in Fig. 3.9 the pack with $10 \%$ walnut shows a high overall displacement, greater than $18 \%$ mixture and almost equivalent to the $25 \%$ walnut mixture. This is contradictory to the expectation that the addition of softer particles should reduce the overall pack stiffness and result in a larger platen displacement. Even in the small scale models with 60 uniformly sized particles (section 2.2.1) it was observed that the response stiffened for packs with a higher fraction of ceramic particles. 
For the models in section 2.2.1 the initial particle placement prior to compressive loading was uniform for each mixture composition. This is impossible to maintain for the polydisperse pack where the initial pack is obtained by allowing the randomly distributed particles to fall freely under gravity. Notice the highly varying particle arrangement of the top layer of particle pack for each of the mixtures in Fig 3.3b, 3.5, 3.6 and 3.7 .

To further elaborate on the effect of top particle arrangement on the pack deformation let us consider Fig. 3.10a, 3.10b and 3.10c. These three figures show the von Mises stress contours of the $10 \%$ by weight walnut mixtures at three load increments during the analysis at pressures of 0.18, 3.2 and 9.4 MPa respectively. Also note that at these three increments the non-dimensional platen displacements are 0.0392 , 0.11 and $0.161 \mathrm{~mm} / \mathrm{mm}$ respectively. It can be observed that for the first two increments at even low pressures significant platen displacement is obtained. Closely observing Fig. $3.10 \mathrm{a}$ and $3.10 \mathrm{~b}$ we notice that the deformation is limited to the softer walnut particles. If we observe the initial configuration we notice that the highest positions of the pack are occupied by the softer walnut particles and these particles are the first to come in contact with the top platen once compression is initiated. The initial stages of pressure vs displacement response are thus dominated by the compression response of a few walnut particles and do not effectively represent the response of the whole pack. At the third increment which is at a pressure of $9.4 \mathrm{MPa}$ we observe that the top layer is now almost flattened, also the stress contours now traverse the entire pack thickness indicating that the load is now getting effectively transmitted from the top platen to the bottom platen. The same observation is repeated as we study the von Mises stress contours for the three load increments of the 25\% walnut mixture, in Fig. 3.11a, 3.11b and 3.11c. In this case also the applied pressure is $0.18,3.2$ and $9.4 \mathrm{MPa}$ respectively while the nondimensional platen displacement is $0.045,0.0975$ and $0.144 \mathrm{~mm} / \mathrm{mm}$ respectively. Again for the first two loads only a few walnut particles seem to deform, while at third increment most of the top layer particles are in contact with the top platen and stress is transmitted from the top platen to the bottom platen. 

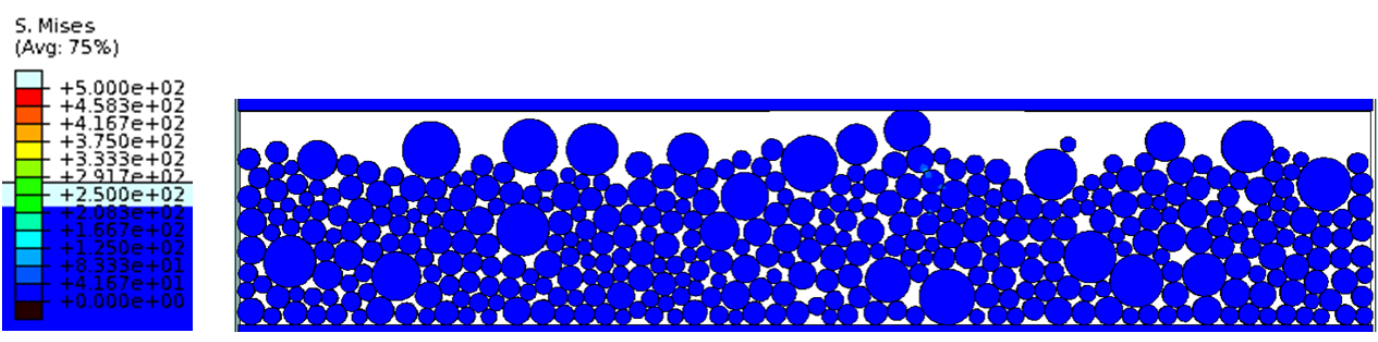

(a)
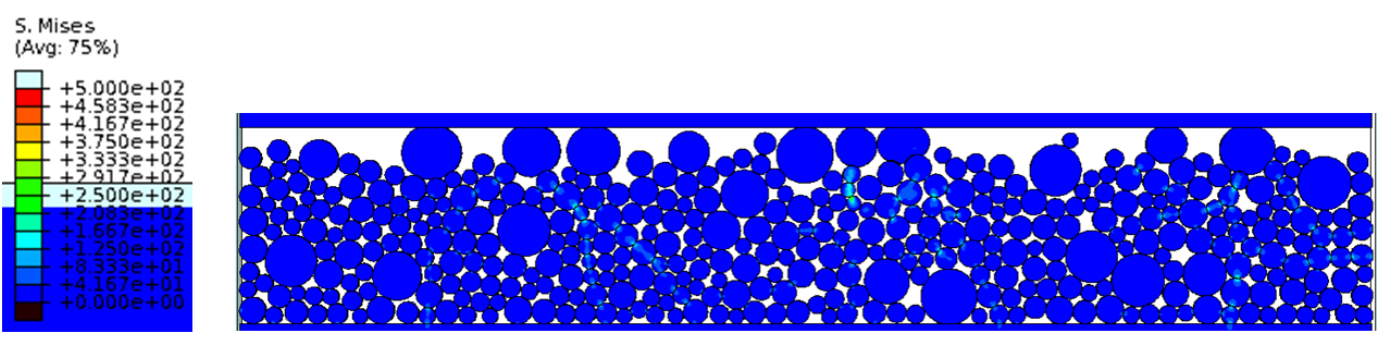

(b)
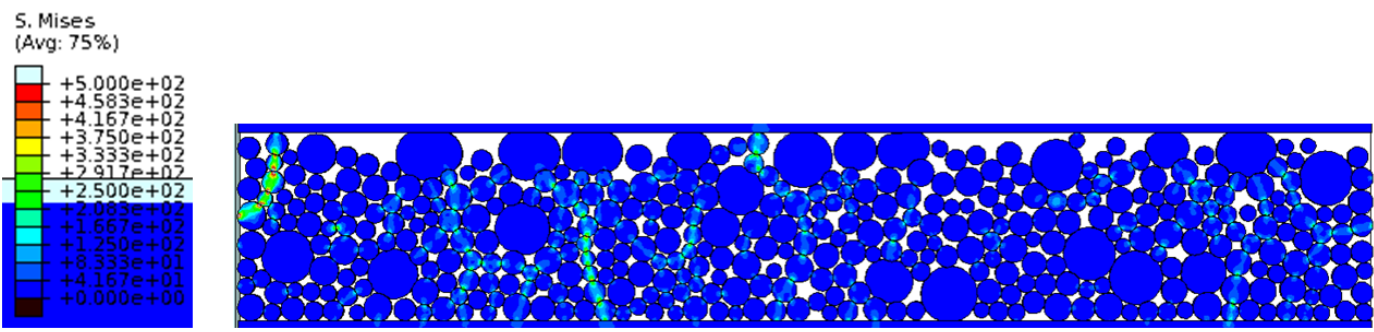

(c)

Fig. 3.10. Von Mises stress contour for 10\% walnut - ceramic mixture at (a) $0.18 \mathrm{MPa}$ pressure (b) $3.2 \mathrm{MPa}$ pressure and (c) $9.4 \mathrm{MPa}$ pressure 

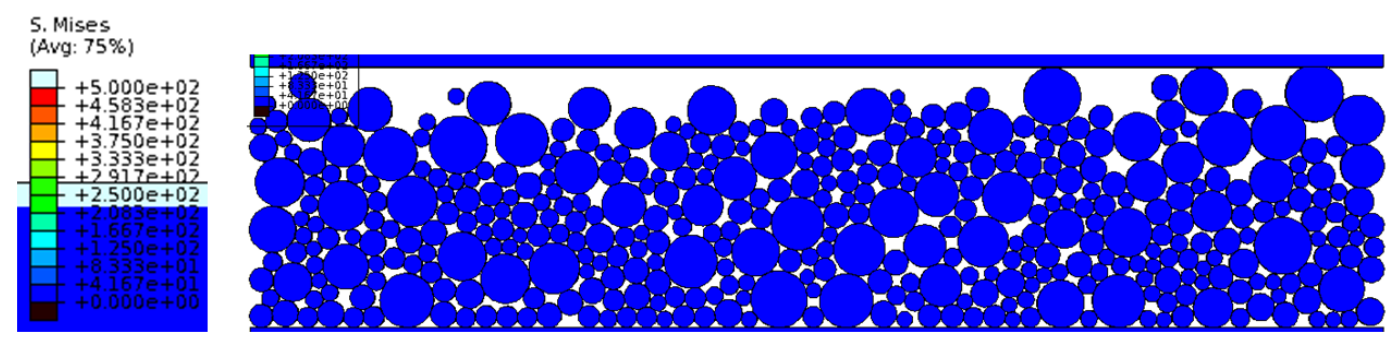

(a)
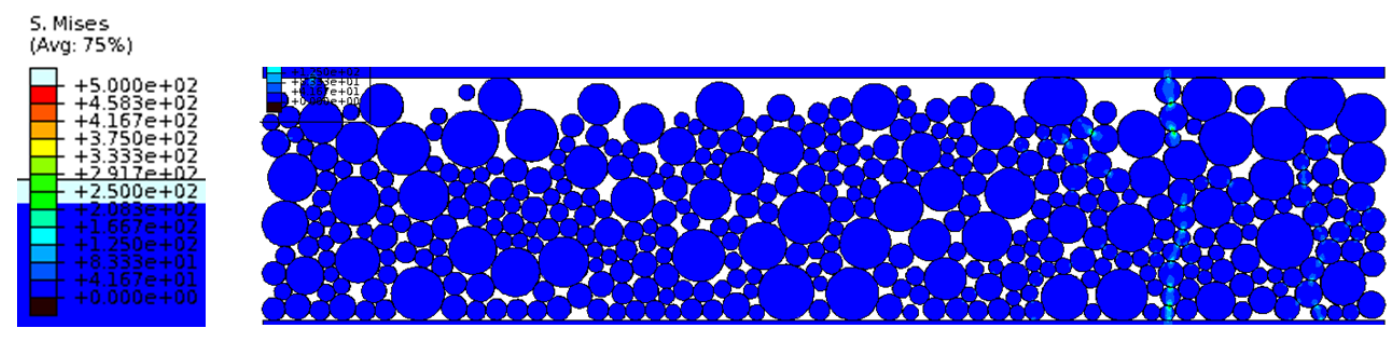

(b)
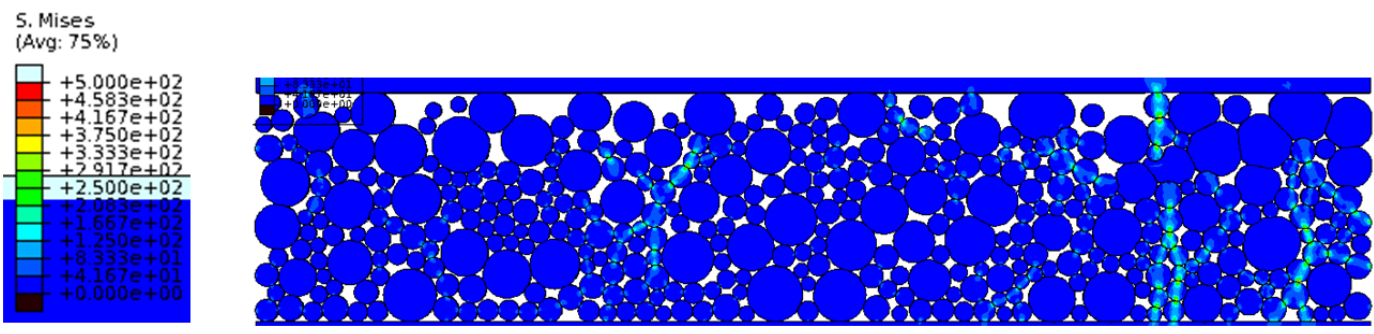

(c)

Fig. 3.11. Von Mises stress contour for 25\% walnut - ceramic mixture at (a) $0.18 \mathrm{MPa}$ pressure (b) 3.2 MPa pressure and (c) 9.4 MPa pressure

During the confined compression crush tests which are discussed later in section 4.2, a uniform top layer is maintained and a preload is applied to the pack before the actual compression is initiated. These two steps ensure that during compression the effect of just the particles at the top layer is minimized and the load vs displacement response represents the overall pack response. The same approach needs to be applied to the computational model. If we consider the value of preload as the pressure at which most of the particles in the top layer are in contact with the platen and stress is getting 
transmitted to the bottom platen then we get preload pressure as $9.4 \mathrm{MPa}$. The plot of pressure vs non-dimensional displacement for all the 4 mixtures assuming a preload of 9.4 $\mathrm{MPa}$ is presented in Fig. 3.12. We now observe that the results are consistent with our expectations and the stiffness of the curve reduces as the percentage of walnut in the mixture increases.

As explained in section 2.2 the image of the deformed particle configuration is first converted to an eight bit black and white image in the image analysis software Image J. The deformed configuration and a black and white image of the processed image for the $10 \%$ walnut mixture at $57 \mathrm{MPa}$ pressure are presented in Fig. 3.13a and $3.13 \mathrm{~b}$. Then the total number of black and white pixels is counted. Void fraction is defined as the ratio of number of black pixels to the total number of pixels in the rectangular domain. The variation of void fraction as a function of pressure for each composition is presented in Fig. 3.14. The hard particles demonstrate larger void fractions at the same applied pressure.

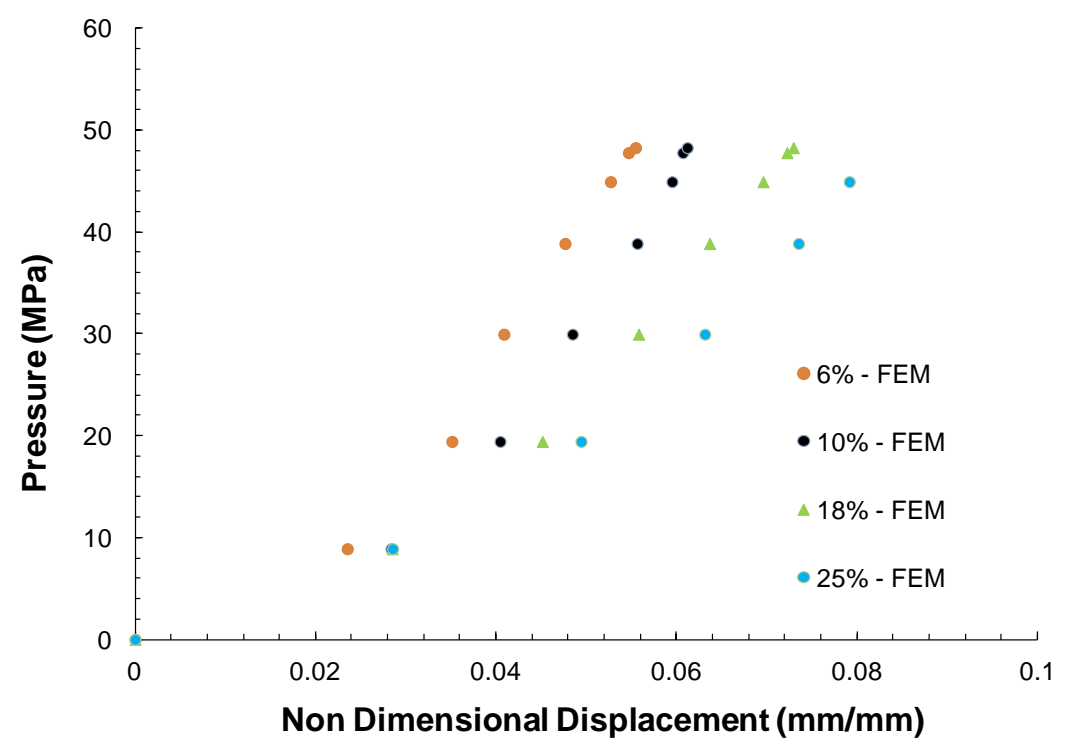

Fig. 3.12. Type-A mixtures, $\mu=0.3$, Pressure vs displacement after preload 


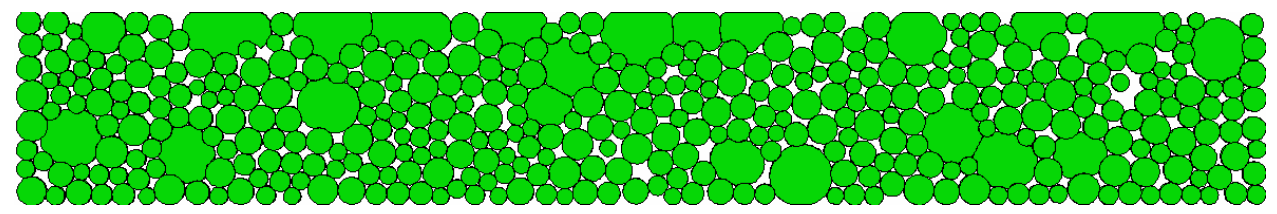

(a)

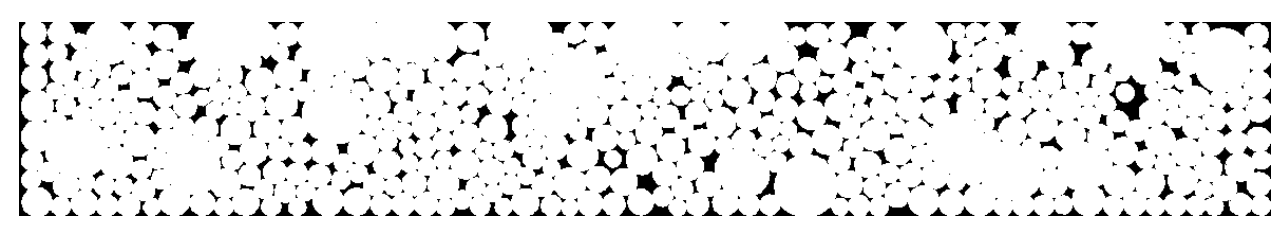

(b)

Fig. 3.13. 10\% walnut mixture at $57 \mathrm{MPa}$ pressure to calculate void fraction (a) Deformed configuration and (b) processed image

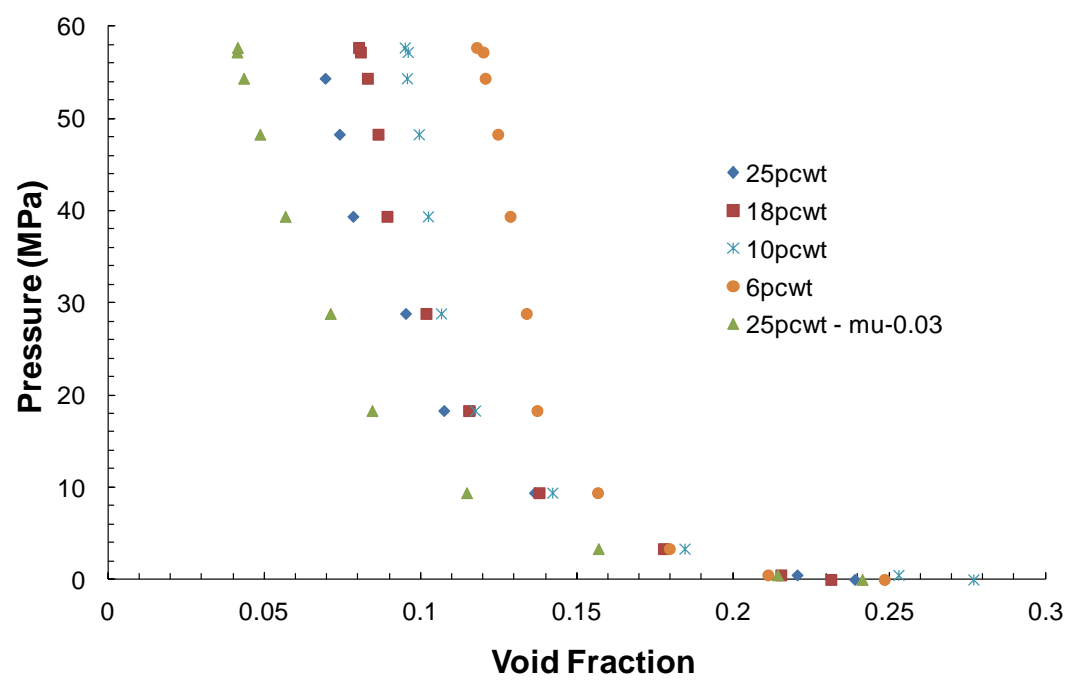

Fig. 3.14. Type-A mixtures, Pressure vs void fraction 
The contour plots for von Mises stress and equivalent plastic strain for the $25 \%$ walnut composition are presented in Fig. $3.15 \mathrm{a}$ and $3.15 \mathrm{~b}$ respectively. It is observed that the stress path passes through the harder ceramic particles while plastic straining is mostly observed in case of the softer particles. It can also be observed that the deformation of the softer particles fills up the void spaces reducing the effective flow area.

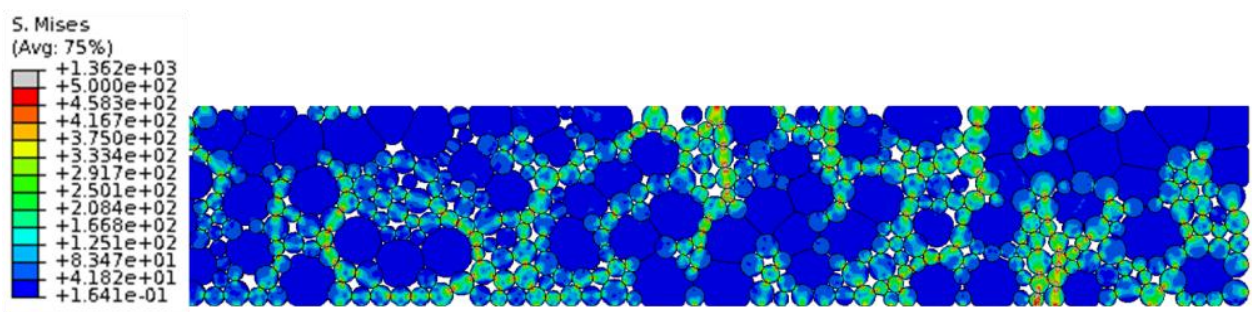

(a)

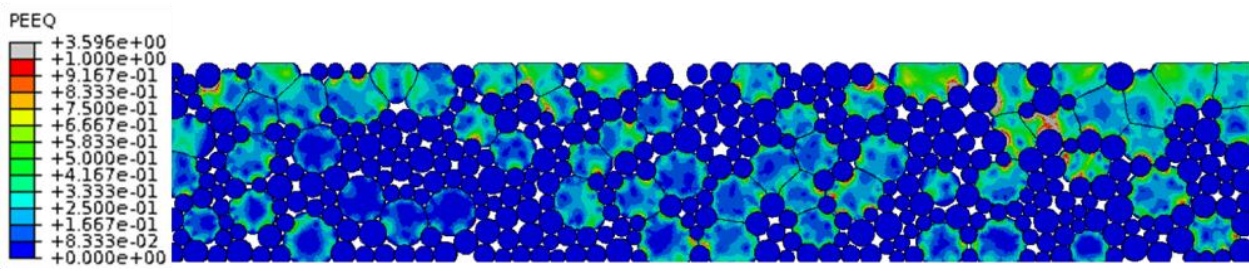

(b)

Fig. 3.15. 25\% walnut at $54 \mathrm{MPa}$ pressure (a) von Mises stress and (b) Equivalent plastic strain

Effect of Material Type and Shape of Soft Particles

The configuration at end of free fall for Type-B mixture at $\mu=0.3$ is presented in Fig. 3.4. The effect of soft/deformable particle material property on pack response is compared by studying the response of two models with different Al material properties, pure $\mathrm{Al}$ and a stronger $\mathrm{Al}$ alloy. A significant increase in porosity is observed with the use of a harder $A l$ alloy compared to pure $A l$, Fig. 3.16. In comparison to walnutceramic mixtures with $25 \%$ walnut composition (Fig. 3.14), the void fraction is much 
higher for Al-ceramic particles. This is attributed to the higher stiffness and strength of aluminum compared to walnut. Aluminum itself carries significant load as observed in the von Mises contour plot in Fig. 3.17a, in contrast to the walnut - ceramic mixtures (Fig. 3.15a) where walnut particles do not carry much load.

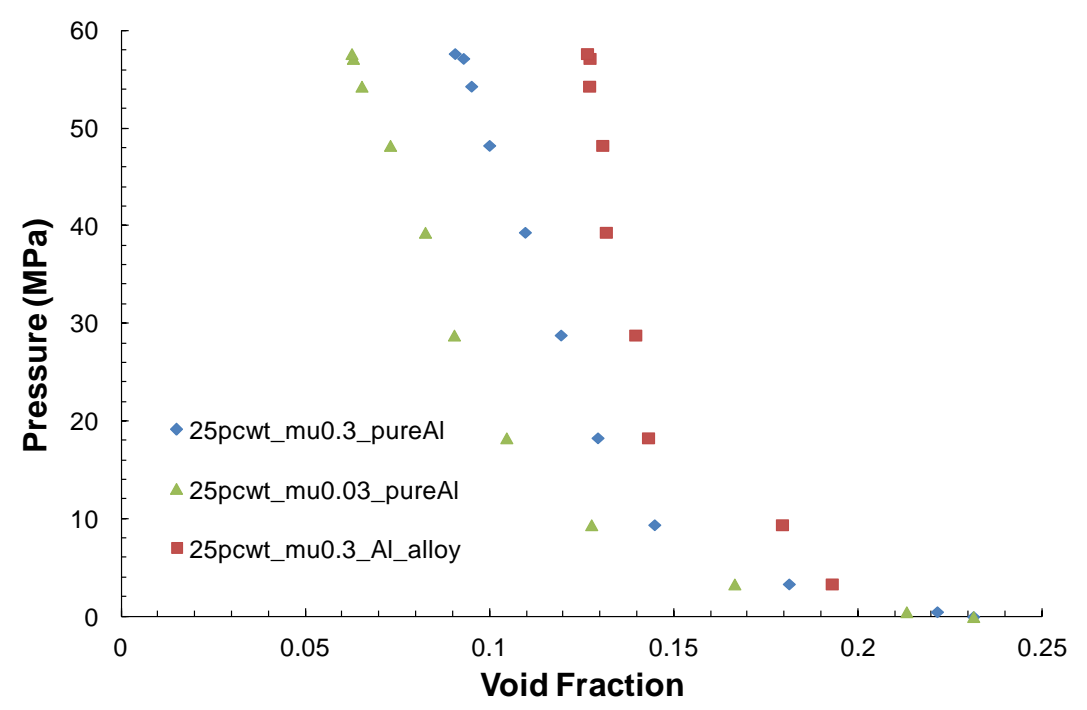

Fig. 3.16. Pressure vs void fraction for Type-B mixture, Al-pure and Al-alloy

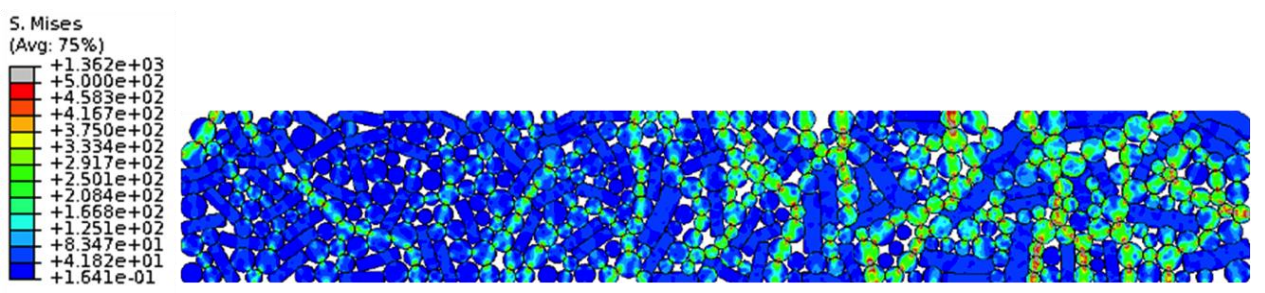

(a)

Fig. 3.17. Von Mises stress for $25 \%$ by weight Type-B mixture at 57 MPa pressure (a) 0.091 void fraction, $\mu=0.3$ and (b) 0.063 void fraction, $\mu=0.03$ 


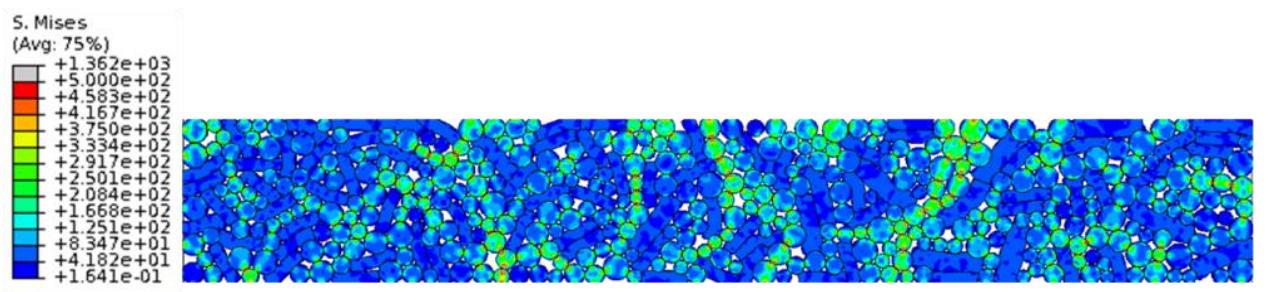

(b)

Fig. 3.17. Continued

Effect of Inter-particle Friction

In both types of mixtures, Type-B and A with $25 \%$ soft particles, the particles undergo greater rearrangement and deformation when friction is lower as resistance to sliding is reduced. In case of Type-A (walnut - ceramic) mixture at $54 \mathrm{MPa}$ applied pressure for the cases with $\mu=0.3$ and 0.03 respectively, the void fraction reduces from 0.069 to 0.043 a reduction of $37 \%$, two leftmost curves in Fig. 3.14. For Type-B (pure aluminum - ceramic) mixture at $54 \mathrm{MPa}$ pressure the void fraction reduces from 0.095 to 0.065 a reduction of $31 \%$, two leftmost curves in Fig. 3.16. Comparing von Mises stress contour plots for Type-B mixture at $\mu=0.3$ and 0.03 in Fig. 3.17, it is noted that particles are much more evenly stressed when friction is lower while high stress concentrations are observed with higher $\mu$. This is because lower friction allows for a greater and easier rearrangement of the pack as it gets compressed thus transmitting the stress more evenly through the particles.

\section{Comparison of Number of Particles in Models}

The von Mises stress contour for the $25 \%$ Type-A 850 particle model at $58 \mathrm{MPa}$ is presented in Fig. 3.18. We can observe the stress path to pass through the ceramic particles while the walnut particles show high deformation. The pressure vs non- 
dimensional displacement response of this model is compared to the 400 particle, TypeA model in Fig. 3.19. The response is very similar with the 850 particle model being a little stiffer at higher loads. The time of solution for the 850 particle model was $50 \mathrm{cpu}$ hours on an IBM p5-575 Cluster, while the 400 particle model took $20 \mathrm{cpu}$ hours. As the difference in the response of the two models is small and it takes much longer to complete the simulation of the larger model we will continue all the future simulations with the 400 particle model.

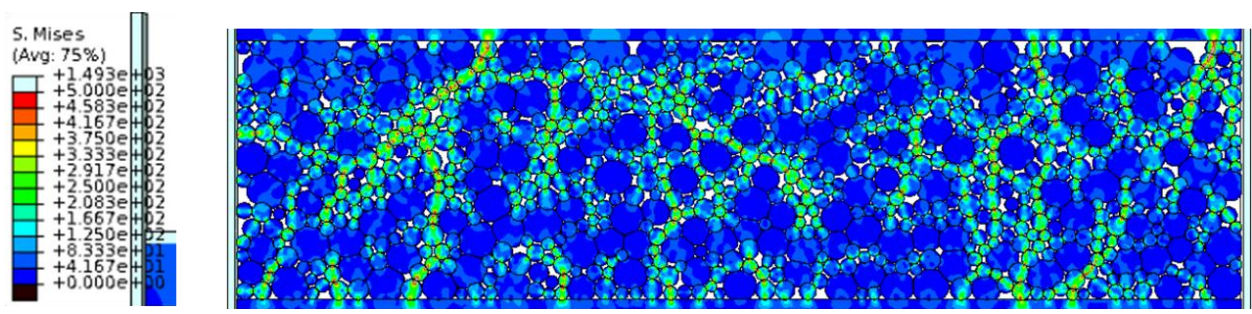

Fig. 3.18. Von Mises stress contour at $58 \mathrm{MPa}$ stress for the 850 particle, $25 \%$ walnut model 


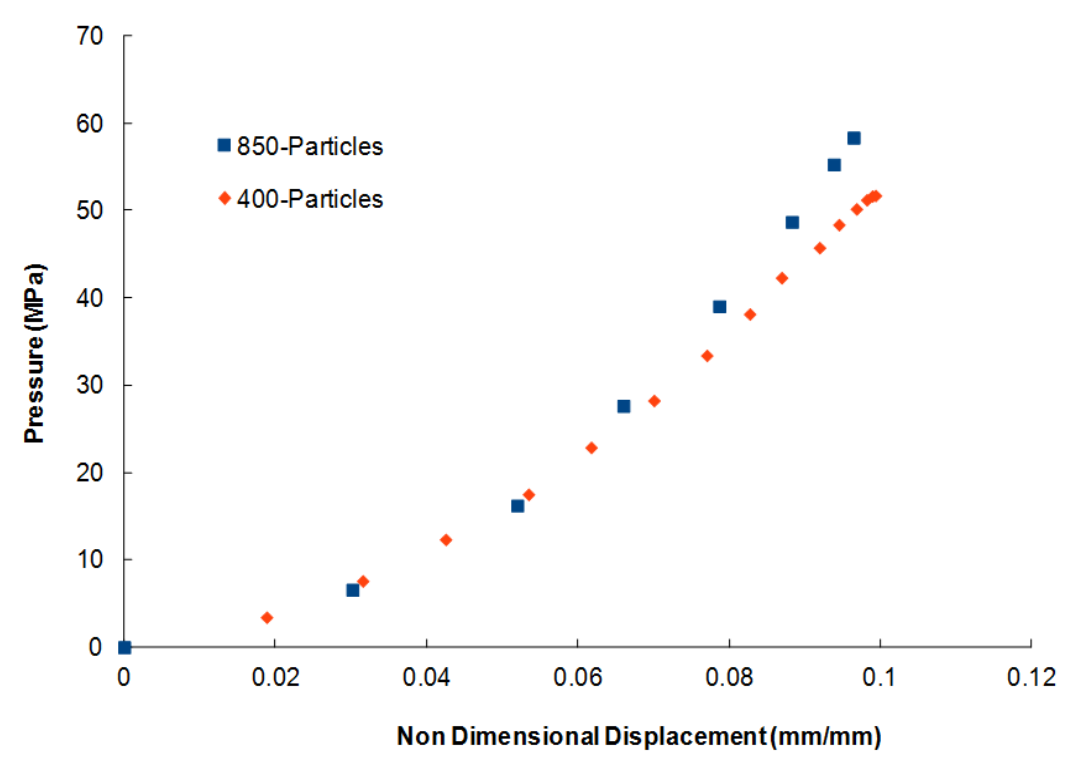

Fig. 3.19. Pressure vs Non dimensional displacement plot comparing Type-A, 25\% by weight walnut response for 400 and 850 particle models

\subsection{Incorporating Particle Fragmentation}

In all the models presented so far, ceramic is assigned the concrete damage plasticity behavior; i.e. isotropic damaged elasticity combined with isotropic tensile and compressive plasticity described in further detail in APPENDIX - B. We now present models where ceramic is considered as a linear elastic material until it reaches its tensile strength limit at which point cracking is allowed to occur. Post cracking, the material shows degradation in its tensile behavior while compression remains as linear elastic. The brittle ceramic is modeled using the brittle failure material model for concrete in ABAQUS $v$ 6.8.3, discussed in detail in APPENDIX - B. Note that in both these approaches a crack does not necessarily mean a discrete single opening, rather a crack is replaced by a continuous medium whose tensile properties can undergo degradation [23, 70]. The models and boundary conditions remain the same as discussed in section 3.2; 
the only difference here is the material constitutive property definition of ceramic particles.

Further, particle fragmentation is incorporated by removing the degraded element from the mesh based on a failure criterion. With the element deletion approach the number of elements and nodes in the model at each increment can vary depending on the change in an element's status. Contact surfaces also needs to be defined on the newly exposed surfaces, this aspect is discussed in APPENDIX - A where general contact is discussed. It is to be noted that even though element deletion method is very widely employed in simulations ranging from material forming, machining, impact and vehicle crash it is not the best approach [71-73]. According to literature for dynamic crack propagation element deletion method is unable to match the performance of certain other fracture simulation techniques like interelement method or XFEM (extended FEM) [74]. Its wide applicability is more due to its ease of implementation. Furthermore, in certain applications even when a crack has been formed the structure can still carry compressive loads in such scenarios element deletion can introduce errors in the simulation. In our case we need to implement particle fragmentation as without particles breaking into pieces a softening in the load vs displacement curve for the proppant pack cannot be simulated. Such a softening in the pack response is observed from the confined compression tests on proppant mixtures, Section 4.2. Currently ABAQUS does not have the capability of implementing XFEM or the interelement method for the explicit solver hence element deletion has been employed in our simulations.

We model the post cracking softening response to have a linear variation by specifying the strain $\left(\varepsilon_{0}\right)$ at which stress reduces to zero. According to literature a better approach in modeling post cracking softening is by specifying the stress vs displacement data as this reduces the problem of mesh sensitivity in the results $[23,75]$. Here displacement means crack opening. Under tension the crack opens but some bridging mechanism exists which ensures that its load bearing capacity reduces gradually. The Mode-1 fracture energy $\left(\mathrm{G}_{\mathrm{I}}\right)$ or in other words the fracture toughness of quasi-brittle material like concrete, rocks or certain ceramics needs to be determined experimentally 
$[70,76,77]$. Mode-1 means a crack opening under tension normal to the crack face. For fracture toughness measurement the most crucial and problematic part of specimen preparation is the generation of a sharp crack and measuring its size. It also requires stiff load frames.

We haven't conducted any experiments on ceramic samples to study its post cracking behavior. Therefore two values of failure strain, $0.4 \%$ and $0.8 \%$ are considered to observe its influence on the response of the structure. Note that if the value of $\mathrm{G}_{\mathrm{I}}$ for a certain material is known the failure strain $\varepsilon_{0}$ can be calculated from equation, $\varepsilon_{0}=2 \mathrm{G}_{\mathrm{I}} /\left(\sigma_{\mathrm{f}}^{*} \mathrm{a}\right)[23,74]$. Where $\sigma_{f}$ is the cracking failure stress (180 MPa in our case), $a$ is the characteristic element length in the mesh $(0.038 \mathrm{~mm}$ in our case $)$. Basically this equation enables us to adjust the value of failure strain according to the mesh size and provides an approximate mesh insensitive result for the known values of $\mathrm{G}_{\mathrm{I}}$. For our models with the failure strains of $0.4 \%$ and $0.8 \%$ we are actually specifying the Mode-I fracture energy as $13.68 \mathrm{~N} / \mathrm{m}$ and $27.36 \mathrm{~N} / \mathrm{m}$ respectively. 25\% Type-A mixture of walnut and ceramic is employed for this study.

The pressure vs non dimensional displacement responses of the $10 \%$ and $25 \%$ Type-A models with concrete damage plasticity obtained in section 3.2 are compared to the response of models with concrete brittle cracking with $0.8 \%$ failure strain. Similar comparison is carried out for the $25 \%$ Type-B (pure Al needles with ceramic) mixtures. The number of particles showing failure is also compared for each of the three models.

\subsubsection{Results and Discussion}

\section{Effect of Failure Strain on Pack Response}

The pressure vs non-dimensional displacement curves for Type-A, 25\% by weight walnut mixture with 0.4 and $0.8 \%$ failure strain for ceramic particles are presented in Fig. 3.20. 


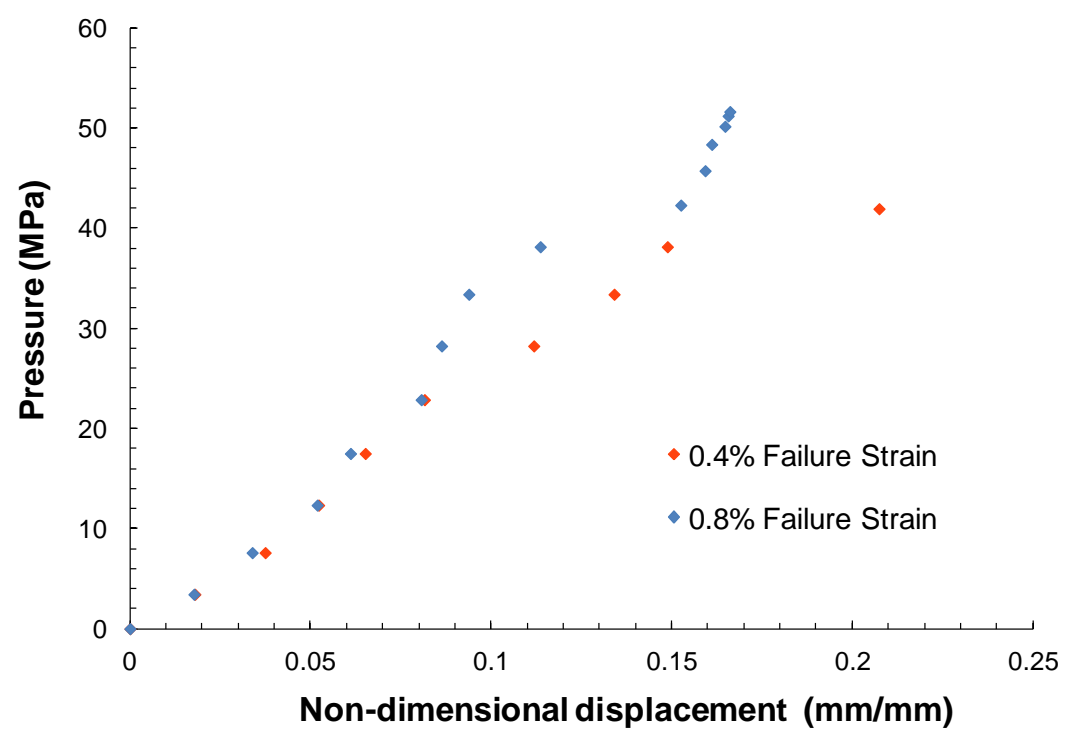

Fig. 3.20. Pressure vs Non dimensional displacement plot comparing Type-A, $25 \%$ by weight walnut response for 0.4 and $0.8 \%$ failure strain, brittle cracking model

The main effect of changing the failure strain is a change in softening behavior of the curve; at the lower loads the response for both cases is similar. Differences arise at larger loads and the $0.8 \%$ failure strain model provides a more stable response. This can be attributed to the fact that the $0.8 \%$ failure strain element will continue to carry stress longer compared to the $0.4 \%$ model. Thus particle fragmentation will occur earlier for the $0.4 \%$ failure strain model compared to the $0.8 \%$ failure strain model which results in greater softening in its pressure vs non-dimensional displacement response.

Comparing Response for Concrete Damage and Brittle Cracking Models

The Type-A 25\% walnut composition forms the basis for this comparison. The $0.8 \%$ failure strain fracture model is compared to the corresponding concrete damage plasticity model in Fig. 3.21. Recall, as discussed earlier in section 3.2 and 2.2, the pressure vs non-dimensional displacement response for models with ceramic damage 
without particle fragmentation showed two distinct regions, one was initial nonlinear response primarily attributed to particle rearrangement and second was continuous stiffening in response due to particle consolidation. With the introduction of particle fragmentation we still observe the two regions, but a third section is also observed wherein the stiffness of the consolidating curve changes and the response softens. As elements are deleted the load carrying capacity of the remaining particle segments is reduced which is reflected in the sudden change in the response which then shows divergence from the damage model as more and more particles continue to fragment. Similarly, in the simulations with damage once particles begin to carry higher pressures and consolidate they do not undergo significant rearrangement. In the models with fracture the remaining fragments can undergo rearrangement which also contributes to the observed softening in the response. Notice in Fig. 3.21 the sudden change in slope for the model with fracture between pressures of 30 and $38 \mathrm{MPa}$, the corresponding deformed configuration is displayed in Fig. 3.22a and 3.22b respectively.

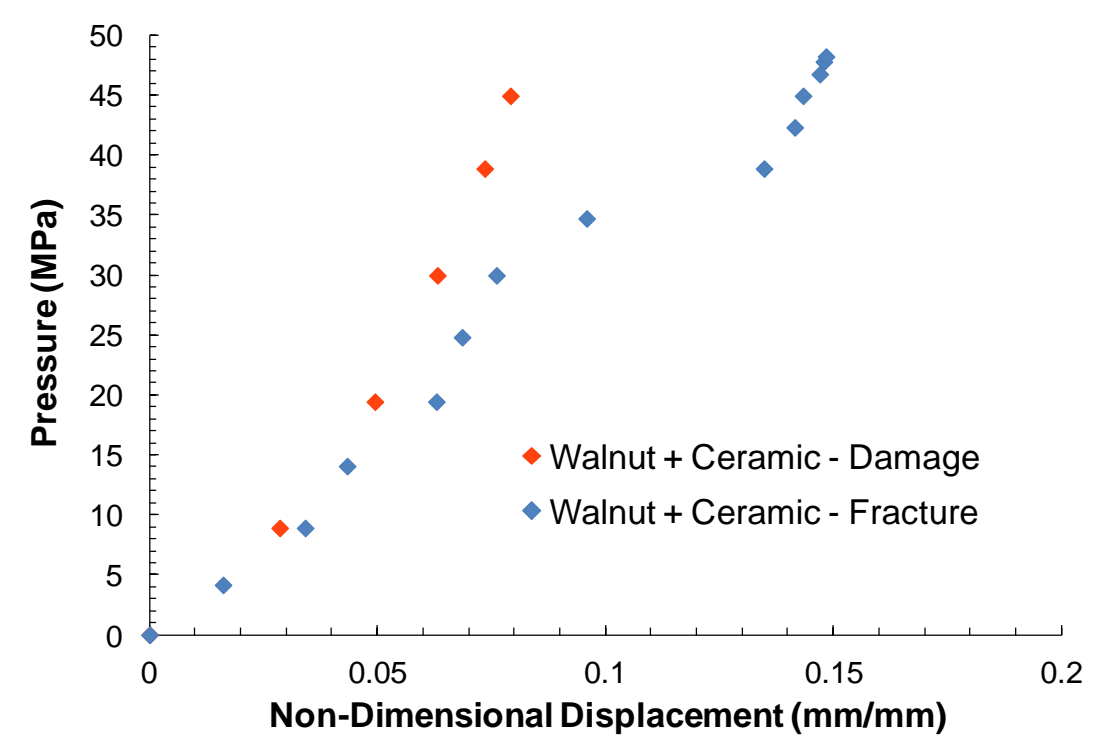

Fig. 3.21. Pressure vs Non dimensional displacement plot for Type-A, 25\% by weight walnut, plastic damage and brittle cracking model comparison 


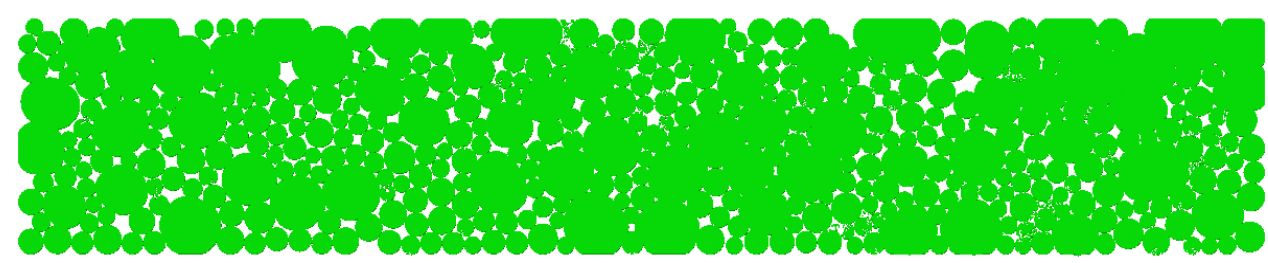

(a)

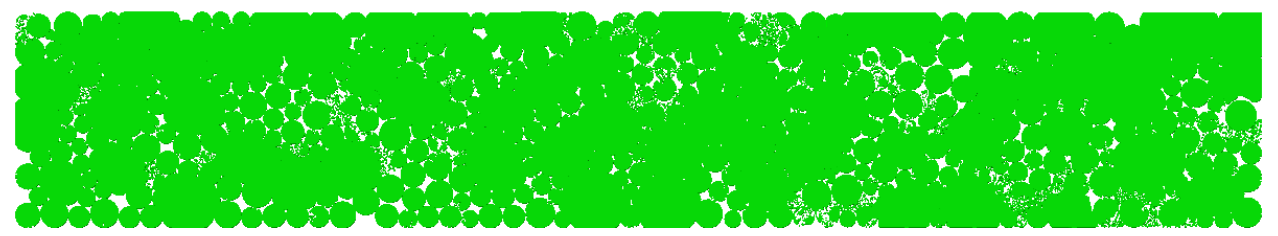

(b)

Fig. 3.22. Configuration of 25\% Walnut, Type-A model at (a) $30 \mathrm{MPa}$ pressure and (b) $38 \mathrm{MPa}$ pressure

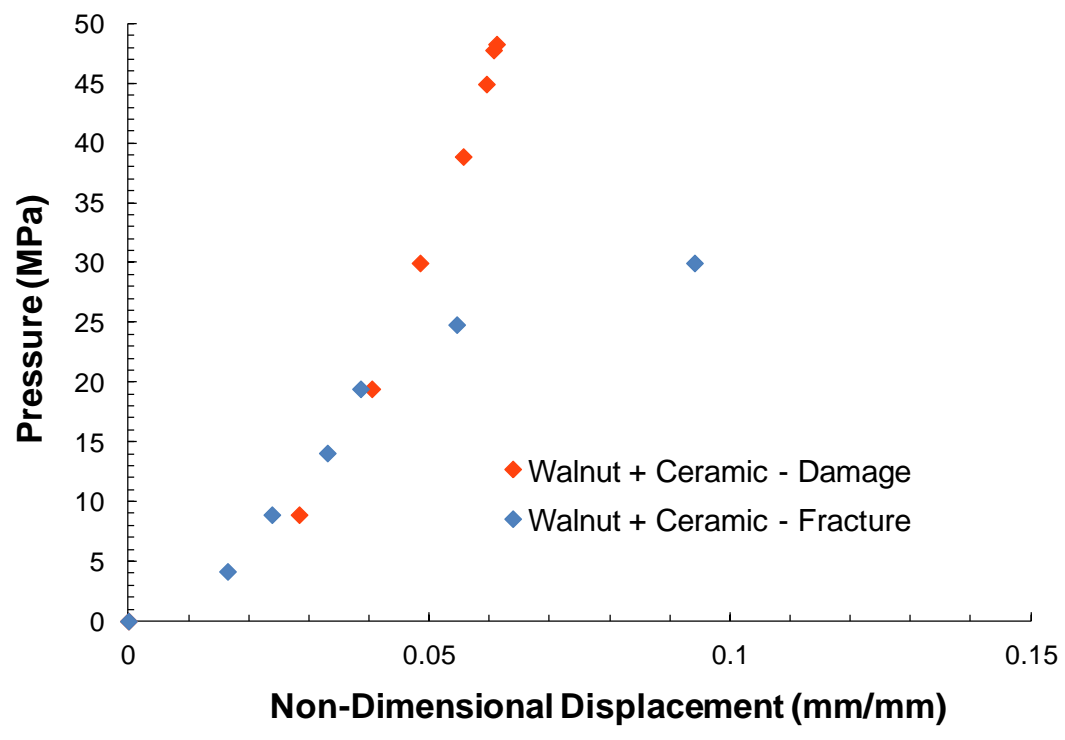

Fig. 3.23. Pressure vs Non dimensional displacement plot for Type-A, 10\% by weight walnut, plastic damage and brittle cracking model comparison 


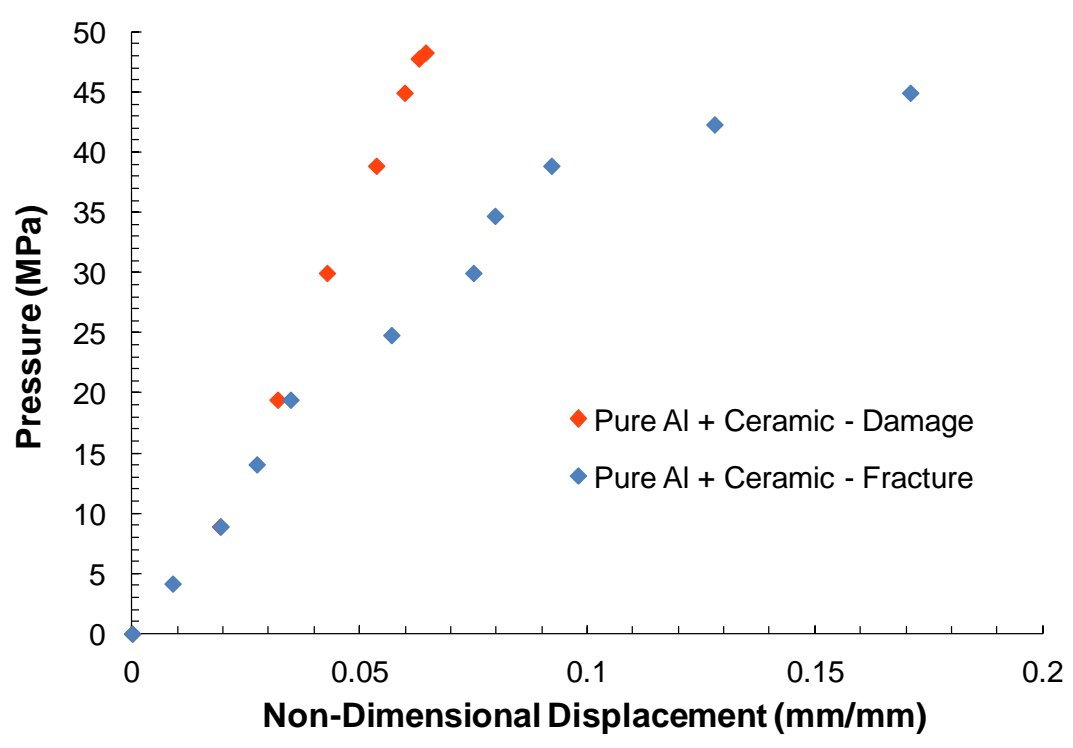

Fig. 3.24. Pressure vs Non dimensional displacement plot for Type-B, 25\% by weight pure Al, plastic damage and brittle cracking model comparison

Similar pressure vs non-dimensional displacement plots for 10\% Type-A mixture and 25\% Type-B (pure $\mathrm{Al}+$ ceramic) mixture comparing the response due to particle fragmentation are presented in Fig. 3.23 and Fig. 3.24 respectively. As with Fig. 3.21 we observe a divergence in the fracture models from the damage models with a decrease in the stiffness of the curve.

\section{Effect of Composition on Response of Brittle Cracking Models}

The pressure vs non-dimensional displacement curves comparing the responses for $10 \%$ and $25 \%$ Type-A (walnut + ceramic) models are presented in Fig. 3.25. At the lower pressures before particle fragmentation assumes significant proportion the $10 \%$ model provides a stiffer response which is similar to our observations for the concrete damage models in section 3.2.2, Fig. 3.12. For the $10 \%$ model particles fracture much earlier at lower pressures compared to the $25 \%$ model. This indicates that the softer 
particles provide cushioning to the ceramic particles and absorb energy while deforming and inhibit ceramic fracture.

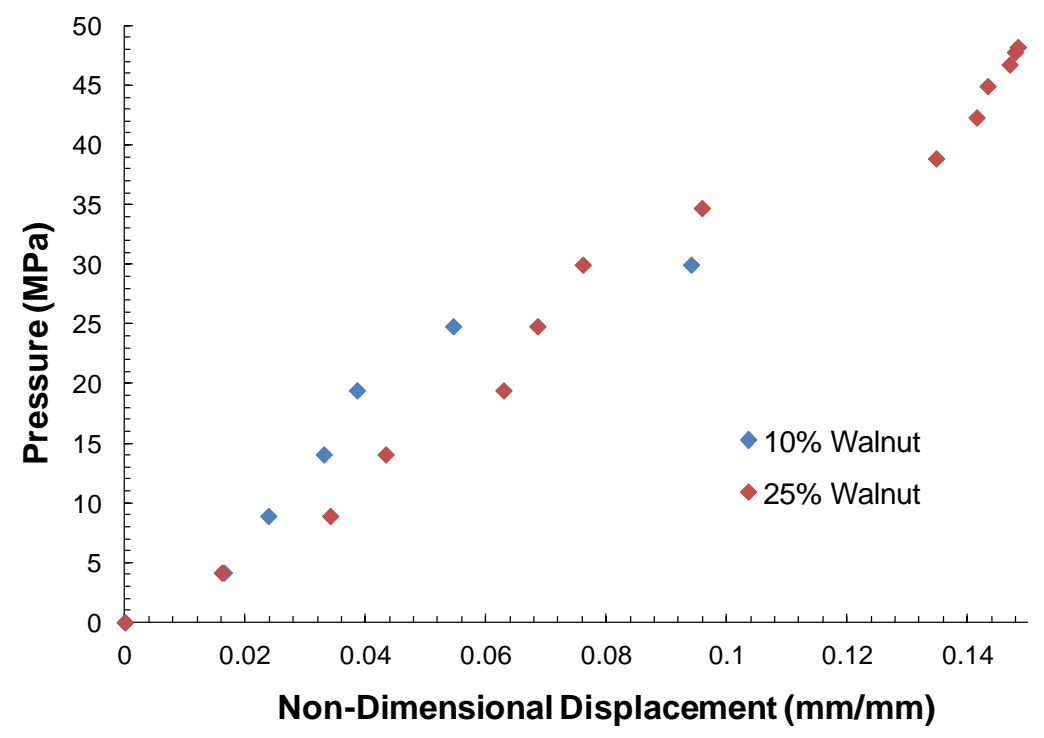

Fig. 3.25. Comparison of $10 \%$ and $25 \%$ walnut mixtures with fracture

We can compare the number of particles fragmenting by observing the element status for the two models at pressure of 25 and $30 \mathrm{MPa}$. This is represented in Fig. 3.26a and 3.26b for both mixtures at $25 \mathrm{MPa}$ and Fig. 3.27a and 3.27b at $30 \mathrm{MPa}$. Note that the two figures show the undeformed configuration and only the element status is plotted with blue indicating deleted element. It can be observed that a much higher proportion of ceramic particle elements are deleted for the $10 \%$ model at $30 \mathrm{MPa}$ which corroborates the observation in Fig. 3.25 where the slope change occurs much earlier for the $10 \%$ model compared to the $25 \%$ walnut model. Also notice the significant increase in the number of deleted particles as pressure increases from $25 \mathrm{MPa}$ to $30 \mathrm{MPa}$ for the $10 \%$ mixture compared to the $25 \%$ mixture. This matches well with the observation in 
Fig. 3.25 where at pressure below $30 \mathrm{MPa}$ the former is stiffer compared to the later but at higher pressures it loses its stiffness due to particle fracture.

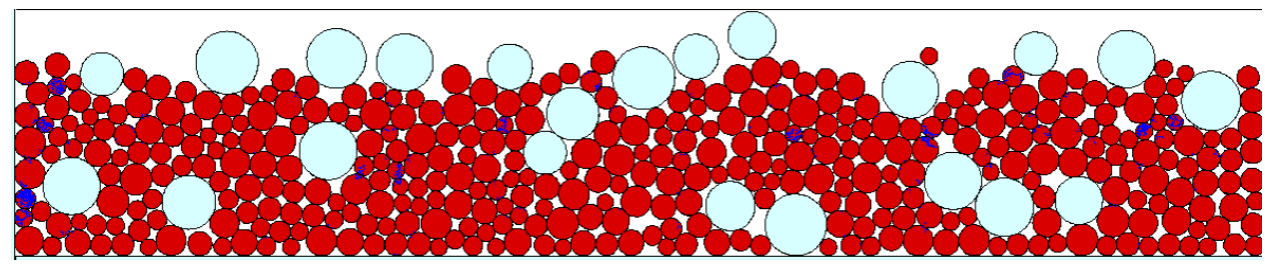

(a)

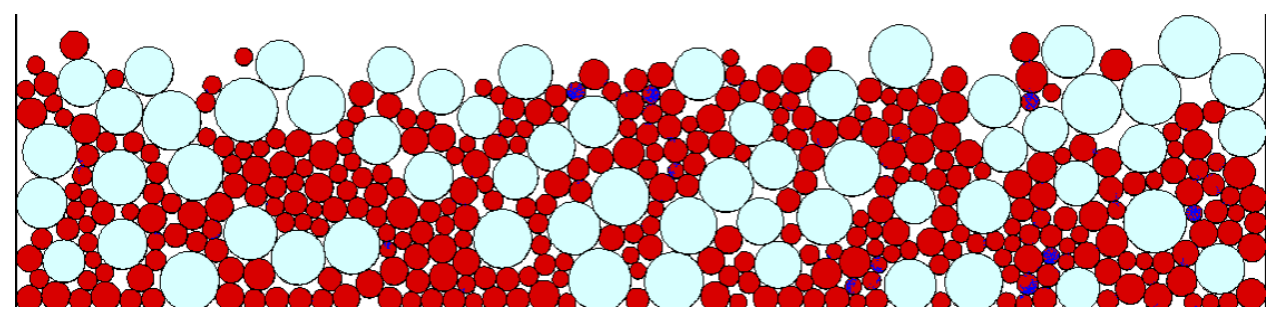

(b)

Fig. 3.26. Comparison of element status at $25 \mathrm{MPa}$ pressure for (a) $10 \%$ walnut ceramic mixture and (b) $25 \%$ walnut ceramic mixtures 


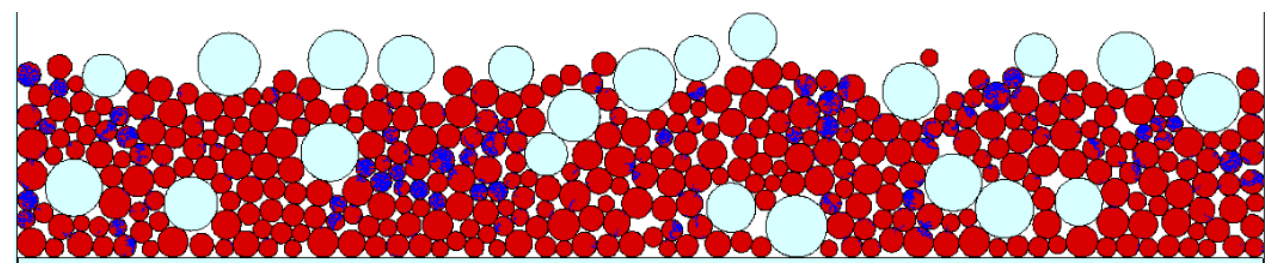

(a)

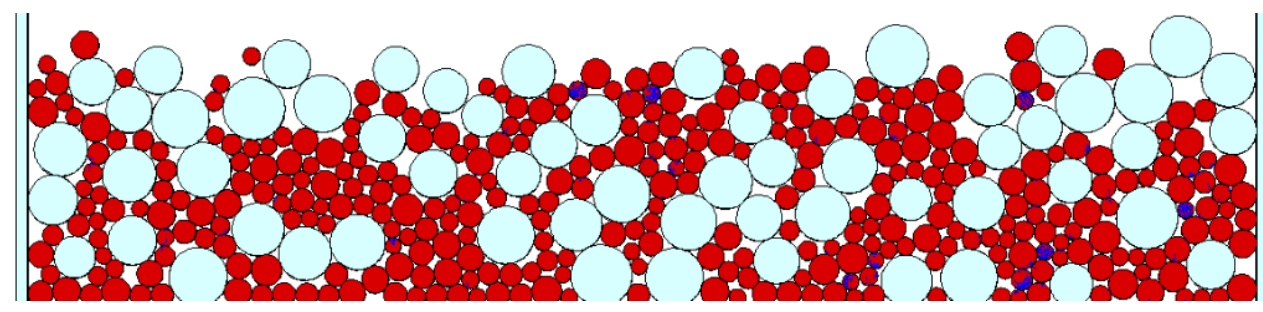

(b)

Fig. 3.27. Comparison of element status at $30 \mathrm{MPa}$ pressure for (a) $10 \%$ walnut ceramic mixture and (b) $25 \%$ walnut ceramic mixtures

Effect of Deformable Material Type on Response of Brittle Cracking Models

The pressure vs non-dimensional displacement responses for 25\% Type-A (walnut + ceramic) and Type-B (pure Al + ceramic) models are presented in Fig. 3.28. It is observed that up to a pressure of $42 \mathrm{MPa}$ the Type-B mixture provides a stiffer response compared to the Type-A mixture. This is mainly due to the stronger material response of pure Al compared to walnut shell. At pressures beyond $42 \mathrm{MPa}$, the pure $\mathrm{Al}$ mixture shows a sudden change in slope and softens. Again comparing the element statuses at $48 \mathrm{MPa}$ pressure for both the mixtures (Fig. 3.29) we observe that the Type-A walnut mixture shows lower fracture compared to the Type-B mixture which explains the observed response. 


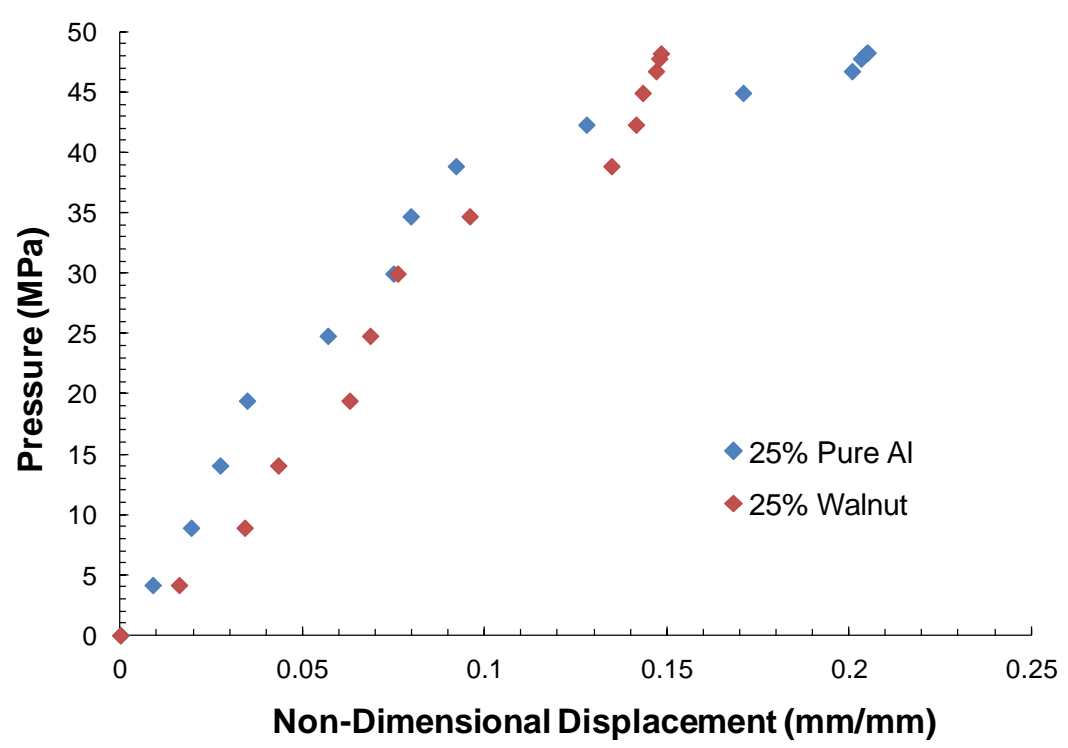

Fig. 3.28. Comparison of 25\% Type-A and Type-B mixtures with fracture

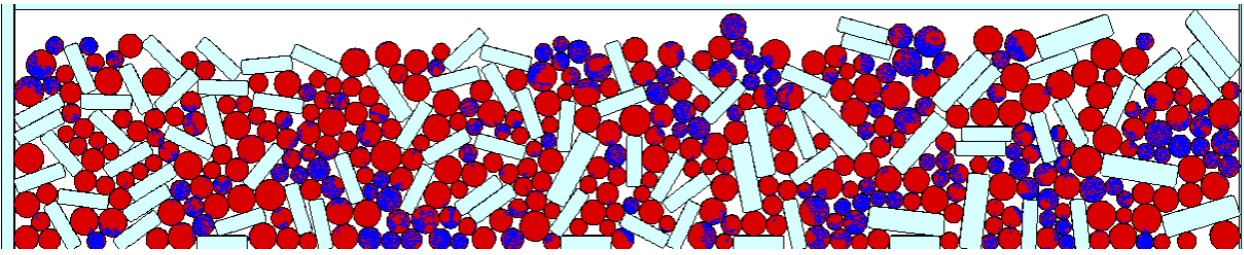

(a)

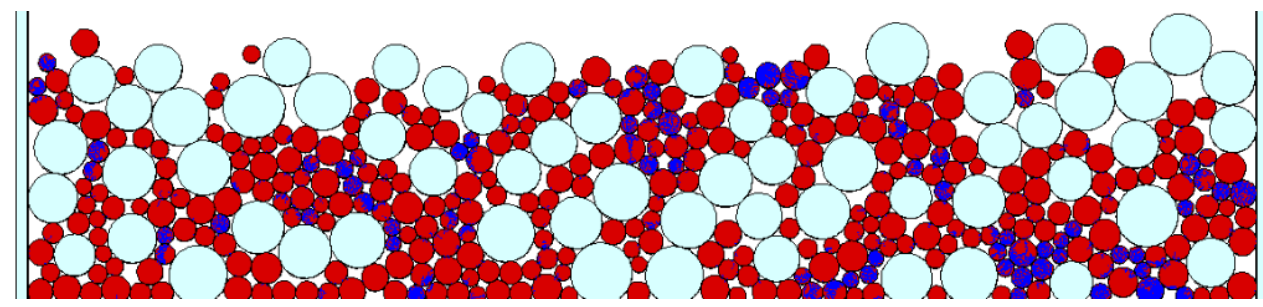

(b)

Fig. 3.29. Comparison of element status at $48 \mathrm{MPa}$ pressure for (a) $25 \%$ pure $\mathrm{Al}$ ceramic mixture and (b) 25\% walnut - ceramic mixtures 


\section{EXPERIMENTAL APPROACH}

Single particle compression and confined compression (crush tests) on proppant samples are reported in this section. The single particle tests on two types of ceramic particles (light weight and high strength) are conducted to study the mechanical response of a single ceramic particle and determine its fracture strength. This data is then incorporated in the FEM models as part of ceramic material property definition. The tests on walnut and pistachios are conducted to compare their mechanical response. The single particle response is also related to the crush test results of proppant mixtures.

Confined compression (crush tests) have been carried out on different proppant mixtures to study the effect on crush resistance and pack stiffness due to the addition of deformable particles of different material types and shapes. The deformable particles considered are $\mathrm{Al}$ needles, $\mathrm{Al}$ spheres, walnut shell particles and pistachio shell particles. Primarily we have focused on proppant packs with the light weight ceramic proppants (LWC) as the base material. Few crush tests have also been reported with low strength (Ottawa sand) and high strength (bauxite) proppants employed as base materials. The comparison of FEM models developed in the previous section with the load vs displacement data from the crush tests is presented in section 5. The crush tests enable us to further refine the models.

\subsection{Single Particle Compression Tests}

Single particle compression tests are conducted on samples of LWC ceramic proppants, high strength (HSP) sintered bauxite proppants, polymer coated walnut and polymer coated pistachio particles. The chemical constitution of the LWC proppant consists of $51 \% \mathrm{Al}_{2} \mathrm{O}_{3}, 45 \% \mathrm{SiO}_{2}, 2 \% \mathrm{TiO}_{2}$ and $1 \% \mathrm{Fe}_{2} \mathrm{O}_{3}$, specific gravity 2.6 [78]. The chemical constitution of the HSP proppant is $\mathrm{Al}_{2} \mathrm{O}_{3}-83 \%, \mathrm{SiO}_{2}-5 \%, \mathrm{TiO}_{2}-3.5 \%$, $\mathrm{Fe}_{2} \mathrm{O}_{3}-7 \%$, specific gravity $3.6[78]$. 
Tests are conducted on INSTRON 3345 vertical test bed with a $225 \mathrm{lbf} / 1000 \mathrm{~N}$ load cell. The tests are displacement control with a loading rate of $0.006 \mathrm{in} / \mathrm{min}$ or $0.00254 \mathrm{~mm} / \mathrm{s}$. The tests are terminated at a maximum load of $150 \mathrm{lbf}$. A total of 36 particles are tested for LWC, 33 for HSP and 12 particles are tested for pistachio and walnut samples respectively. Prior to testing each particle is photographed under a microscope and compared with a datum to determine its initial dimensions. For ceramic particles the initial dimension is its diameter as the particles are predominantly spherical. The LWC ceramic and the HSP bauxite particle along with the datum sphere are shown in Fig. 4.1a and $4.1 \mathrm{~b}$ respectively. It has been reported previously that walnut particles can be segregated into three categories depending on their shape; the flat top particles were observed to show the least scatter in the data and were ideal for comparison purposes $[59,60]$. As the primary intention of testing walnut and pistachio particles is to compare their mechanical response flat top particles were selected for testing. The initial dimension involves cross-section area and thickness, thickness is considered as the smallest dimension of a cuboid. Load is applied along the thickness direction perpendicular to the cross-section. It was observed that pistachios predominantly fell in the flat top category. Fig. 4.2a and Fig. 4.2b show the cross-section and thickness of the walnut particles, Fig. $4.3 \mathrm{a}$ and $4.3 \mathrm{~b}$ show the same for pistachio particles. 


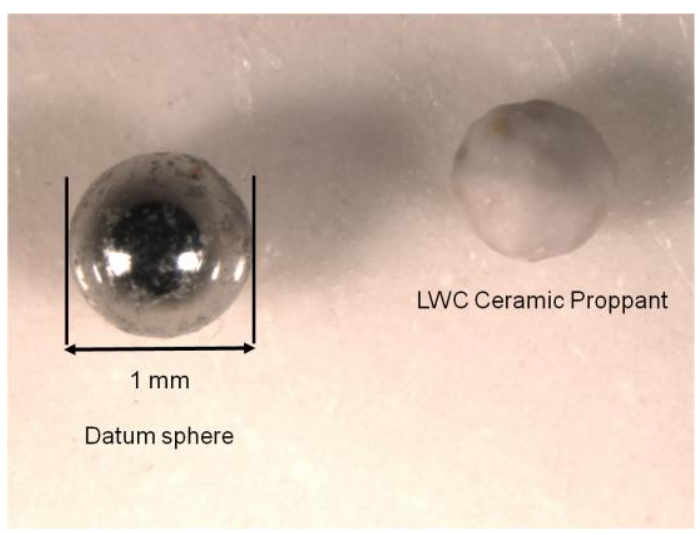

(a)

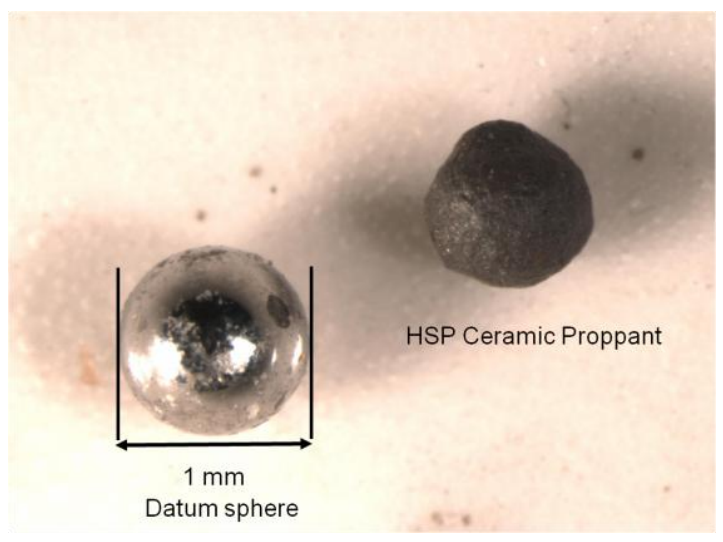

(b)

Fig. 4.1. (a) LWC ceramic proppant and (b) HSP proppant prior to test with the datum sphere

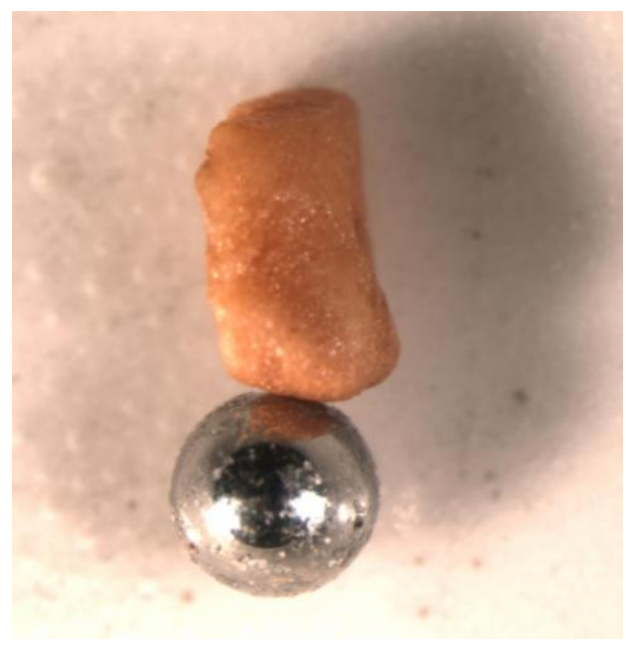

(a)

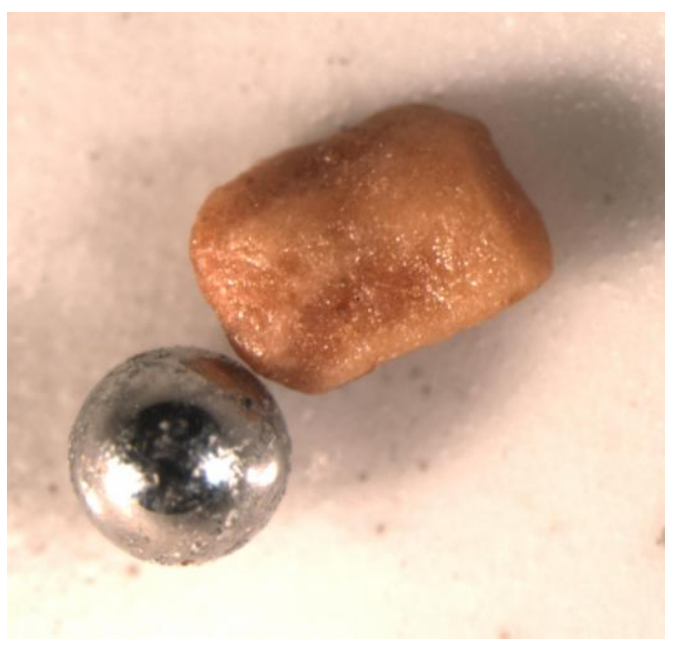

(b)

Fig. 4.2. (a) Walnut proppant with the datum particle highlighting the thickness (b) Same particle along the cross-section highlighting the area 


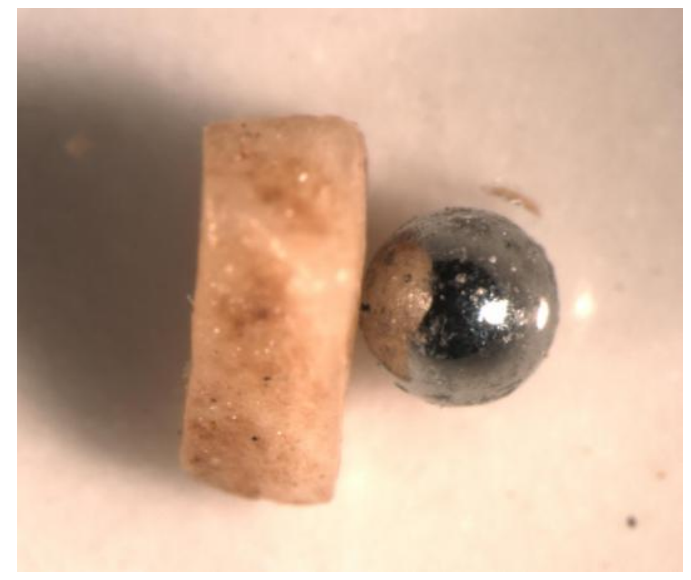

(a)

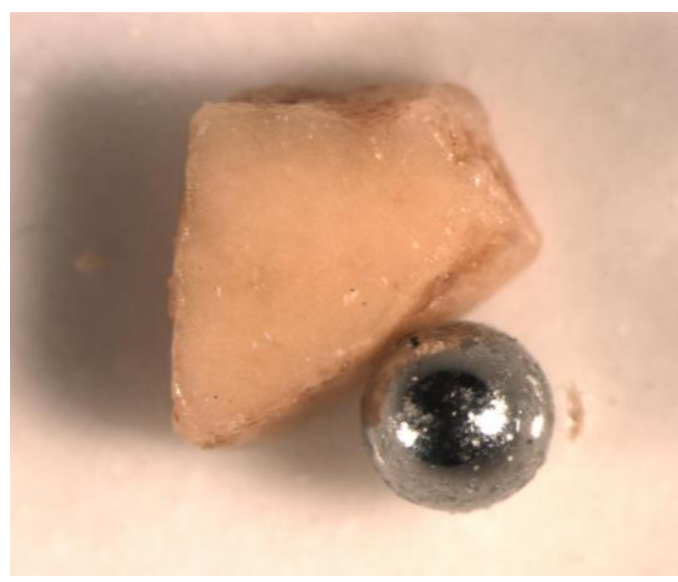

(b)

Fig. 4.3. (a) Pistachio proppant with the datum particle highlighting the thickness (b) Same particle along the cross-section highlighting the area

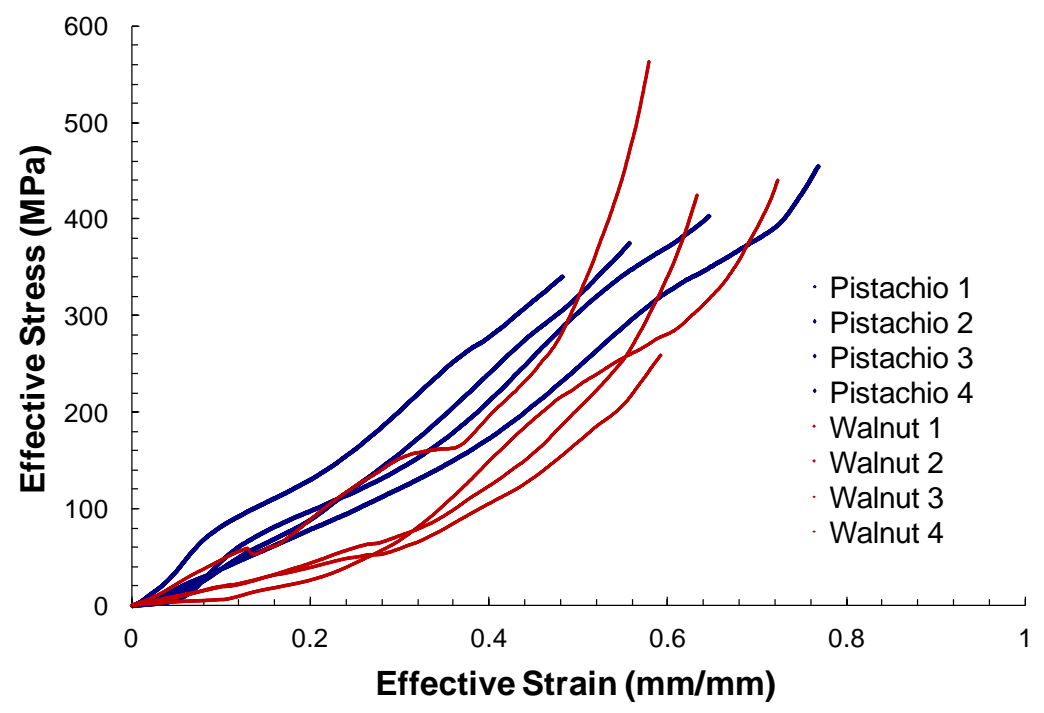

Fig. 4.4. Effective stress vs Effective strain response for single particle compression of coated walnut (maroon) and coated pistachio (blue) 


\subsubsection{Results and Discussion}

\section{Walnut and Pistachio Single Particle}

The effective stress vs effective strain response for the coated flat top walnut and coated pistachio particles is presented in Fig. 4.4. Response for 4 of the 12 tested particles for each type is shown which represents the scatter in the data from least stiff to most stiff response. The load as measured from the load cell is non-dimensionalized with the cross-section area to obtain the effective stress; the crosshead displacement is nondimensionalized with the undeformed particle thickness to obtain the effective strain. The particle dimensions show significant variation, the cross-section area for the pistachios vary from 2.11 to $1.5 \mathrm{~mm}^{2}$ and thickness varies from 0.54 to $0.8 \mathrm{~mm}$. Similarly for walnuts the cross-section area varies from 2.55 to $1.17 \mathrm{~mm}^{2}$ and thickness varies from 0.63 to $0.99 \mathrm{~mm}$. It can be observed that overall the pistachios tend to give a much stiffer response compared to the walnut particles. This can be attributed to their more uniform cuboid shape while the flat top walnut particles showed a slight surface curvature compared to the pistachios. The difference could also be due to different cell structure and chemical composition which needs to be investigated.

\section{Light Weight Ceramic Proppant}

The load vs displacement response for 12 of the total 36 particles tested is plotted in Fig. 4.5a. The diameters for the particles varied from 0.75 to $0.98 \mathrm{~mm}$. The response is primarily linear elastic in accordance with the Hertz contact description of a sphere and a rigid flat. The load at failure is identified as a break in the load vs displacement curve where a sudden steep drop in the force is observed for the displacement controlled loading of a ceramic particle, Fig $4.5 \mathrm{~b}$. This behavior is different from the walnut and pistachio results where the load increases continuously and a break in the curve is not observed. It has been reported in the literature that brittle failure of spherical particles 
initiates at the center of the sphere and the crack extends along the diameter oriented with the loading axis [79]. Fracture is assumed to initiate when the principle stress in tension exceeds the failure stress in tension. The single particle tests on spherical brittle ceramic particles can be used to determine the failure stress for the ceramic material as $\sigma_{\mathrm{f}}=2.8 \mathrm{~F}_{\text {failure }} /\left(\pi \mathrm{D}^{2}\right)[58,79]$. Here $\sigma_{\mathrm{f}}$ is the failure stress in tension, $\mathrm{F}_{\text {failure }}$ is the load measured by the load cell at the point of particle fracture and $\mathrm{D}$ is the particle diameter. It can also be observed that there is significant variation in the failure load for each particle which is not uncommon for brittle material like ceramics. The Weibull statistical analysis is employed to characterize the mechanical response for the ceramic particles [58].

According to the procedure as described in [58] for each of the 36 particles tested we get a specific diameter, failure load and hence tensile strength. These 36 data points are then sorted in an ascending order of their strength. A probability of failure is defined for each particle as $\mathrm{Pf}_{\mathrm{i}}=(\mathrm{i}-0.5) / \mathrm{N}$, where $\mathrm{i}$ ranges from 1 to 36 and $\mathrm{N}=36$. A plot of $\ln \left(\ln \left(1 /\left(1-\mathrm{Pf}_{\mathrm{i}}\right)\right)\right)$ vs $\ln \left(\sigma_{\mathrm{f}}\right)$ is then constructed, Fig. 4.6. By applying a linear fit to this plot we can obtain Weibull modulus $(\mathrm{m})$ and characteristic strength $\left(\sigma_{0}\right)$ for the material based on the equation $\ln \left(\ln \left(1 /\left(1-\mathrm{Pf}_{\mathrm{i}}\right)\right)\right)=\mathrm{m} \ln \left(\sigma_{\mathrm{f}} / \sigma_{0}\right)$. A higher value of $\sigma_{0}$ points to a higher material strength and a higher value of Weibull modulus (m) imply a better reliability of the predicted strength data. For the current 36 single particle tests the characteristic strength is obtained as $159.11 \mathrm{MPa}$ while the Weibull modulus is calculated to be 4.729 . At the same time the average strength is calculated as 149.39 MPa with a standard deviation of $24.73 \mathrm{MPa}$. 


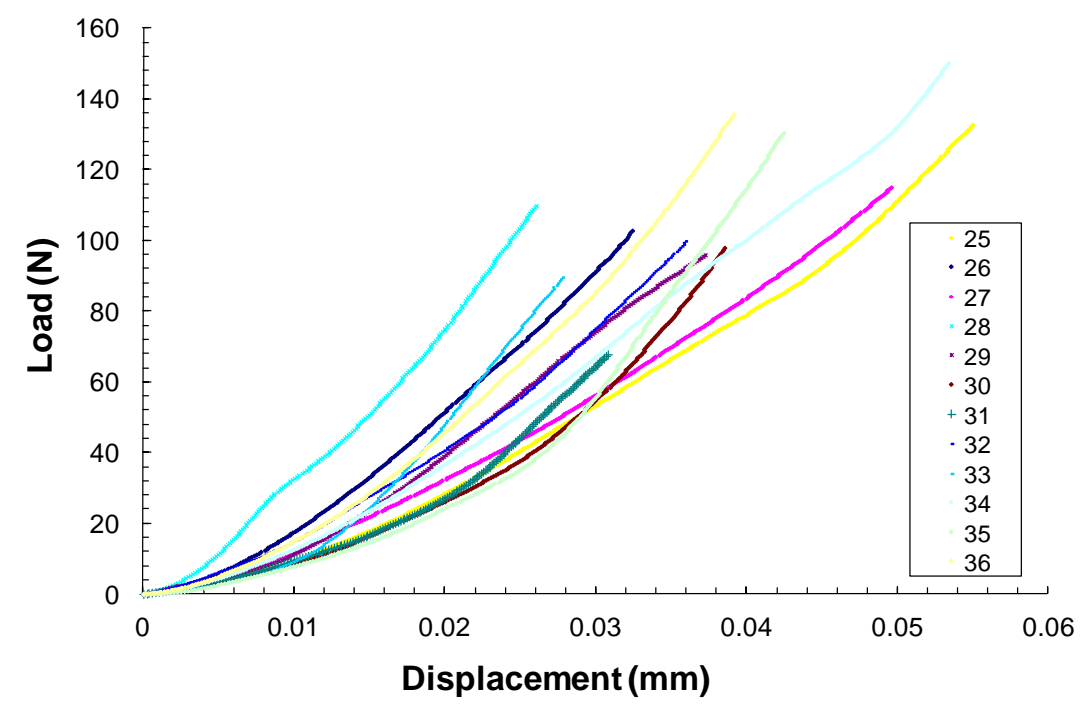

(a)

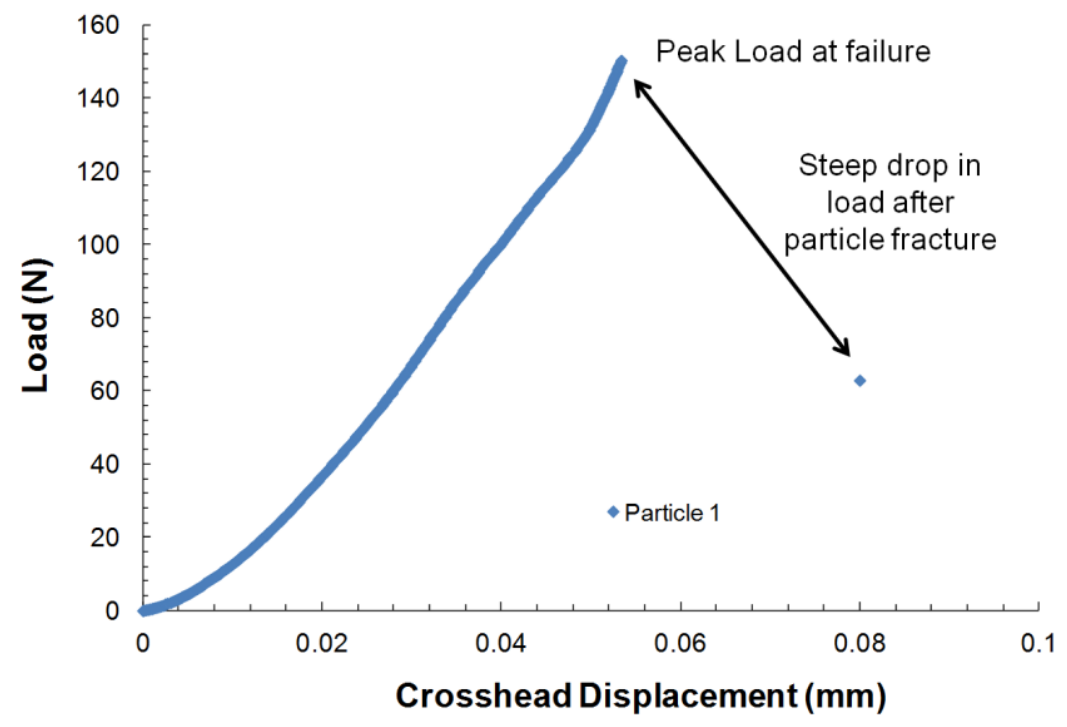

(b)

Fig. 4.5. (a) Load vs Displacement response for 12 of the 36 LWC ceramic particles (b) Load vs Displacement for a single particle highlighting the peak load at failure and drop in load after fracture 


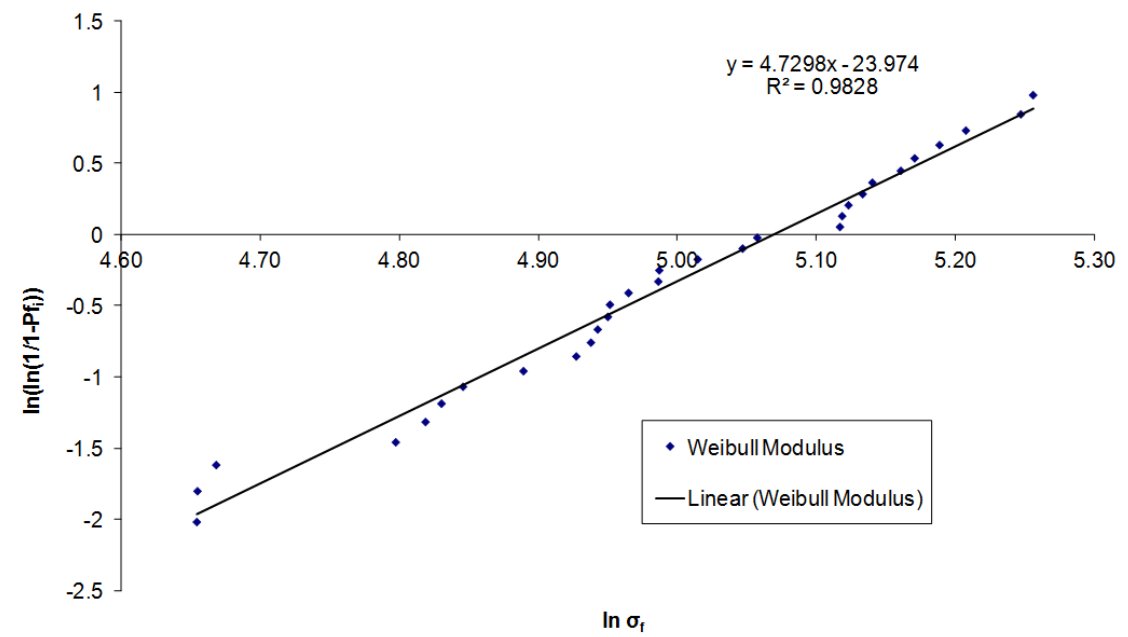

Fig. 4.6. $\ln \left(\ln \left(1 /\left(1-P f_{i}\right)\right)\right)$ vs $\ln \left(\sigma_{f}\right)$ plot with a linear fit to obtain Weibull modulus for LWC proppants

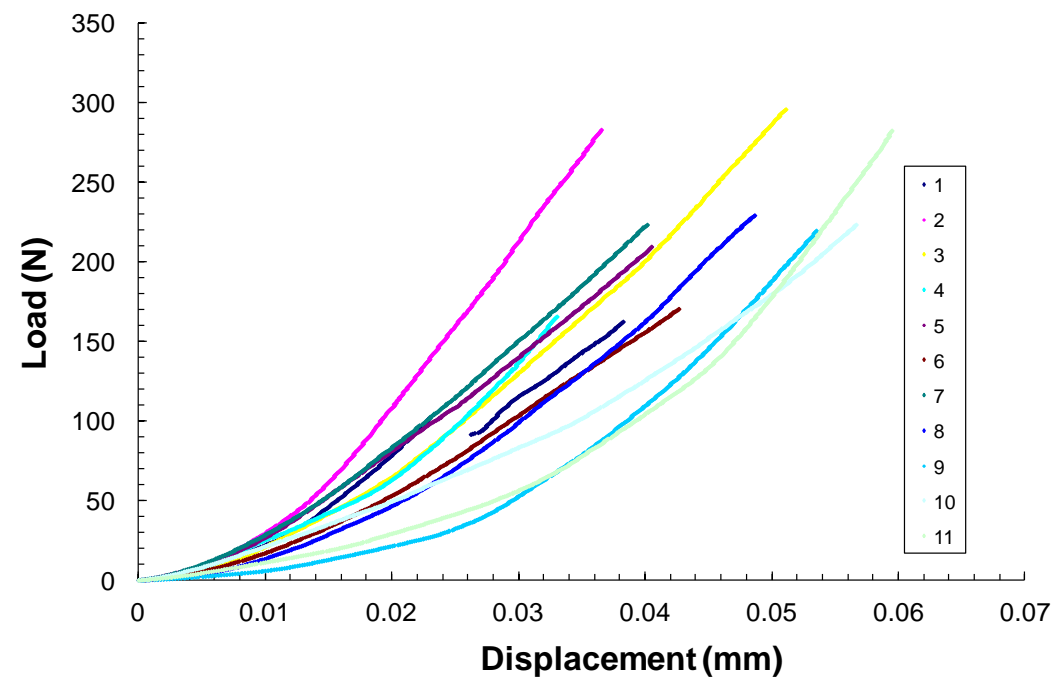

Fig. 4.7. Load vs Displacement response for 12 of the 33 HSP ceramic particles 


\section{High Strength Proppant}

The load vs displacement responses for 12 high strength proppants are presented in Fig. 4.7. The diameters for the particles ranged from 0.74 to $0.91 \mathrm{~mm}$. Here also we observed a linear elastic response followed by brittle fracture. Compared to the LWC particles the fracture load is higher. In this case also Weibull statistical analysis is employed to characterize the fracture strength of the particles. Fig. 4.8 presents the $\ln \left(\ln \left(1 /\left(1-\mathrm{Pf}_{\mathrm{i}}\right)\right)\right)$ vs $\ln \left(\sigma_{\mathrm{f}}\right)$ plot. The characteristic strength of this proppant is calculated as $298.94 \mathrm{MPa}$, the Weibull modulus is 8.054. Both these values are much higher compared to the ISP proppants, indicating a much stronger material and better reliability in the prediction of the strength. The higher strength is primarily attributed to the composition of the material with $83 \% \mathrm{Al}_{2} \mathrm{O}_{3}$ for $\mathrm{HSP}$ proppant compared to $51 \%$ for the LWC proppant.

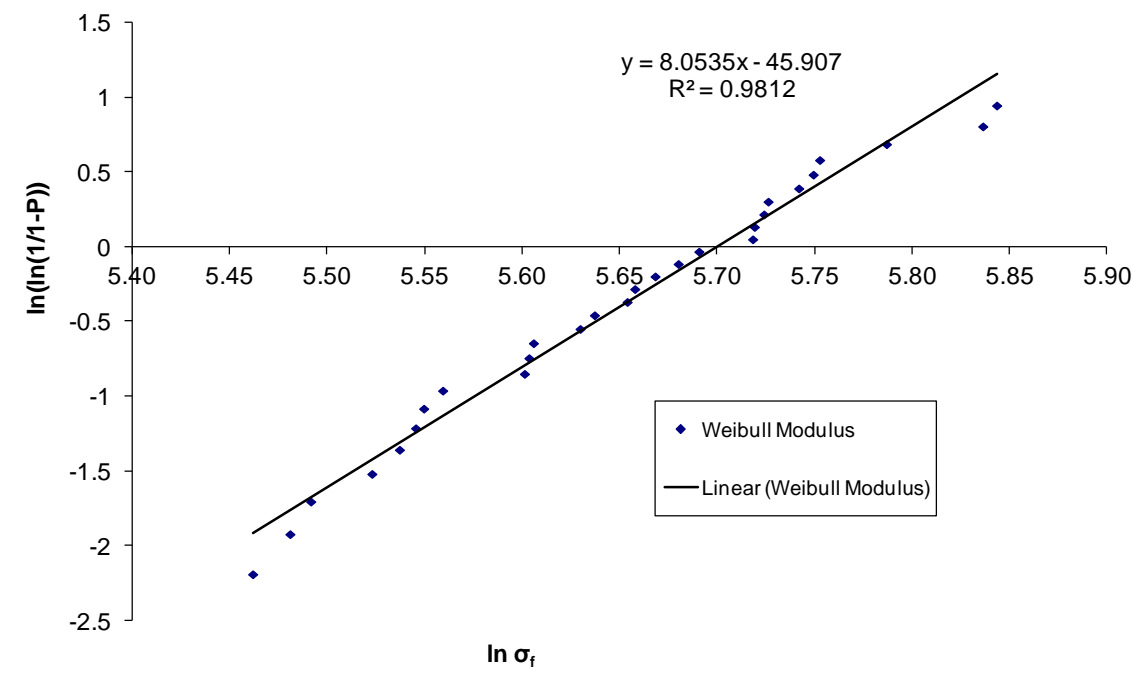

Fig. 4.8. $\ln \left(\ln \left(1 /\left(1-\mathrm{Pf}_{\mathrm{i}}\right)\right)\right)$ vs $\ln \left(\sigma_{\mathrm{f}}\right)$ plot with a linear fit to obtain Weibull modulus for HSP proppant particles 


\subsection{Crush Testing}

Fines formed due to the crush/fracture of the brittle proppants occupy the interparticle porosities and are severely detrimental to oil/gas production. Crush testing conducted in accordance with the API RP 60 standards is a standard industrial test procedure to evaluate the strength of a proppant pack. These tests represent stress state on proppants deep within a fracture away from the well bore; they can also be termed as confined compression tests. In case of proppant mixtures the brittle particles fragment and form smaller particles. Crush tests are evaluated based on the weight of sample with size less than mesh 40 or 400 microns remaining at the end of the test. We have also obtained the load vs displacement response from the tests to evaluate the effective pack stiffness. In the present study three base materials have been considered LWC, HSP and Ottawa sand. Deformable particles are added to these baseline materials and the effect on crush and pack stiffness is evaluated. The sand + walnut mixtures and the HSP $+\mathrm{Al}$ needle mixtures are commercial products employed in the industry. The sand + walnut mixtures are generally employed at pressures below 7500 psi while the HSP + Al needles mixtures are employed at high pressures of 15000 psi.

\subsubsection{Equipment and Materials}

The crush cell has a diameter of 2.033 in with a wall thickness of 0.5 in and height of $3 \mathrm{in}$. The loading piston has a diameter of crush cell diameter minus $0.005 \pm$ $0.001 \mathrm{in}$. The height of the piston is $3.5 \mathrm{in}$. The piston material is 4340 alloy steel with minimum hardness of Rockwell C 43. These specifications are in accordance with the API RP 56 and 60 standards [7, 8]. Tests are conducted on a MTS load frame with a load cell capacity of $250,000 \mathrm{lbs}$ calibrated to an accuracy of $250 \mathrm{lbs}$. Spherical seats are used on the compression platens to ensure proper alignment while loading. Load is applied hydraulically and is computer controlled. The crush cell with the loading piston mounted on the load cell is shown in Fig. 4.9. As the granular pack is compressed in the 
crush cell the piston displacement is measured with an LVDT (linear variable differential transducer) calibrated to an accuracy of 0.001 in.

As stated previously three different proppant types are considered as base materials, low strength (Ottawa sand), light weight ceramic and high strength. The chemical constitution of the LWC proppant consists of $48 \% \mathrm{Al}_{2} \mathrm{O}_{3}, 48 \% \mathrm{SiO}_{2}, 2 \% \mathrm{TiO}_{2}$ and $1 \% \mathrm{Fe}_{2} \mathrm{O}_{3}$, specific gravity is 2.6 [78]. The chemical constitution of the HSP proppant includes $\mathrm{Al}_{2} \mathrm{O}_{3}-83 \%, \mathrm{SiO}_{2}-5 \%, \mathrm{TiO}_{2}-3.5 \%, \mathrm{Fe}_{2} \mathrm{O}_{3}-7 \%$, specific gravity is 3.6 [78]. The bulk density of Ottawa sand and the LWC ceramic proppant is $1.56 \mathrm{~g} / \mathrm{cm}^{3}$ and $41.15 \mathrm{~g}$ particles are used in each test, the particle size ranges between sieve \# 40 and 20 or $425-850$ microns. For the HSP proppants the bulk density is $2.0 \mathrm{~g} / \mathrm{cm}^{3}$ and $50.0 \mathrm{~g}$ particles are used in each test. These quantities are in accordance with the API RP 60 test standards. The baseline tests are followed by tests on proppant mixtures where deformable particles are added to the base proppant. We have primarily focused on the LWC proppant mixtures, the different mixtures considered, particle sizes and percent weight of soft particles added are summarized in Table. 4.1. In all cases the total weight of particles used in a test is kept constant at $41.15 \mathrm{~g}$.

For the HSP proppants we have considered $10 \%$ and $25 \%$ mixtures of Al needles and $\mathrm{Al}$ spheres, for the tests $50.0 \mathrm{~g}$ sample is used. For the low strength Ottawa sand proppants $10 \%$ mixtures of walnut and pistachio are considered and sample weight is $41.15 \mathrm{~g}$ for each mixture during the test. 


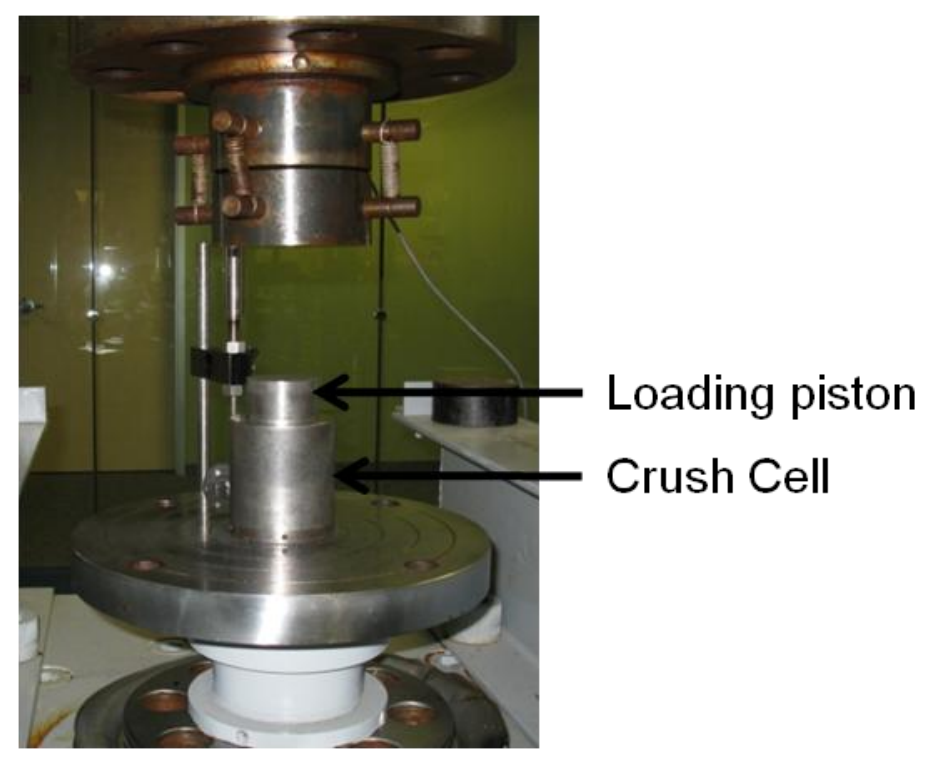

Fig. 4.9. Crush cell and loading piston mounted on the load frame

Table. 4.1. Material, size range and concentration of deformable particles considered in mixtures. The base material is LWC ceramic proppant. Total sample weight is $41.15 \mathrm{~g}$

\begin{tabular}{|c|c|c|c|}
\hline No. & Material & Size range (microns) & $\%$ weight added \\
\hline 1 & Walnut shells & $1180-600$ & $6,10,18,25,33$ \\
\hline 2 & Pistachio shells & $1180-600$ & $6,10,18,25,33$ \\
\hline 3 & Al needles & $(3500-2500) \mathrm{X}(425-850)$ & $6,10,18,25,33$ \\
\hline 4 & Al spheres & $1180-600$ & $6,10,18,25,33$ \\
\hline
\end{tabular}

\subsubsection{Test Procedure}

The first step of the test involves sieving the samples to obtain particles in a specific size range. The ceramic particle's diameter ranges from 425-850 micron (sieve 
\# 40 - 20) while those of the walnut particles range from 600-1180 micron (sieve \# 30 16). Then the particles are mixed evenly and the mixture is poured in the crush cell using a funnel. A loose pack is maintained and the cell is neither jarred nor shaken. The piston is lowered gently on the pack and twisted by 180 degrees to obtain a level surface. The particle layer thickness is measured. A preload of $\sim 75 \mathrm{lbs}$ is applied on the pack prior to actual loading. Loading is force controlled and applied over three steps i) ramp up over 1 minute ii) hold constant for 2 minutes and iii) unloading over 1 minute. During the current tests the maximum pressure is maintained at $68 \mathrm{MPa}(10,000 \mathrm{psi})$ for LWC mixtures, $103 \mathrm{MPa}(15,000 \mathrm{psi})$ for HSP and $51 \mathrm{MPa}(7500 \mathrm{psi})$ for sand. At the end of the test the contents from the crush cell are transferred to a sieve. The sieve is placed on a shaker for 10 minutes to allow for segregation and the weight of crushed particles (diameter smaller than 400 microns) which fall through the \# 40 sieve is measured. An LVDT provides the additional displacement data. Note that the displacement data provided from the LVDT does not account for pack deformation during the 180 degree twist of the loading piston and preload.

\subsubsection{Results and Discussion: LWC Mixtures}

A typical pressure vs non-dimensional displacement response obtained from a crush test is depicted in Fig. 4.10. Pressure is obtained by dividing the load with the crush cell diameter; the displacement is non-dimensionalized by dividing it with the initial loose pack thickness. The figure shows the response obtained during the 1 minute load ramp for the LWC ceramic proppants. These tests form the baseline with which the mixtures are compared. As is shown in Fig. 4.10, the response can be divided into three distinct phases the initial nonlinear response is mainly due to particle rearrangement. The second phase involves consolidation and an approximately linear response, here rearrangement is not significant and the particles start carrying significant load. In this stage some particles start fracturing but their overall influence on the response is not 
significant. The final stage shows softening which is primarily due to a large number of particles fragmenting.

The percentage weight of proppant crushed (fractured) is obtained by first sieving the sample at the end of the test and then weighting the sieved sample with size less the 400 microns. For the baseline tests on LWC ceramic proppants the average percentage crush (weight of fractured particles with size less than 400 microns related to the original sample weight of $41.15 \mathrm{~g}$ ) is obtained as $11.906 \%$ with a maximum of $12.29 \%$ and minimum of $11.23 \%$ with a standard deviation of $0.588 \%$. Effective pack stiffness is obtained by measuring the slope in the linear region of the pressure vs nondimensional displacement (between pressures of 14 and $40 \mathrm{MPa}$ ). The average effective stiffness for the baseline tests is calculated to be $2408 \mathrm{MPa}$ with a standard deviation of 54.4 $\mathrm{MPa}$, the minimum and maximum values are calculated as $2382.2 \mathrm{MPa}$ and 2470.5 $\mathrm{MPa}$ respectively. The data for the average percent crush and effective pack stiffness for the mixtures is presented in Tables 4.2-4.5 along with the standard deviation.

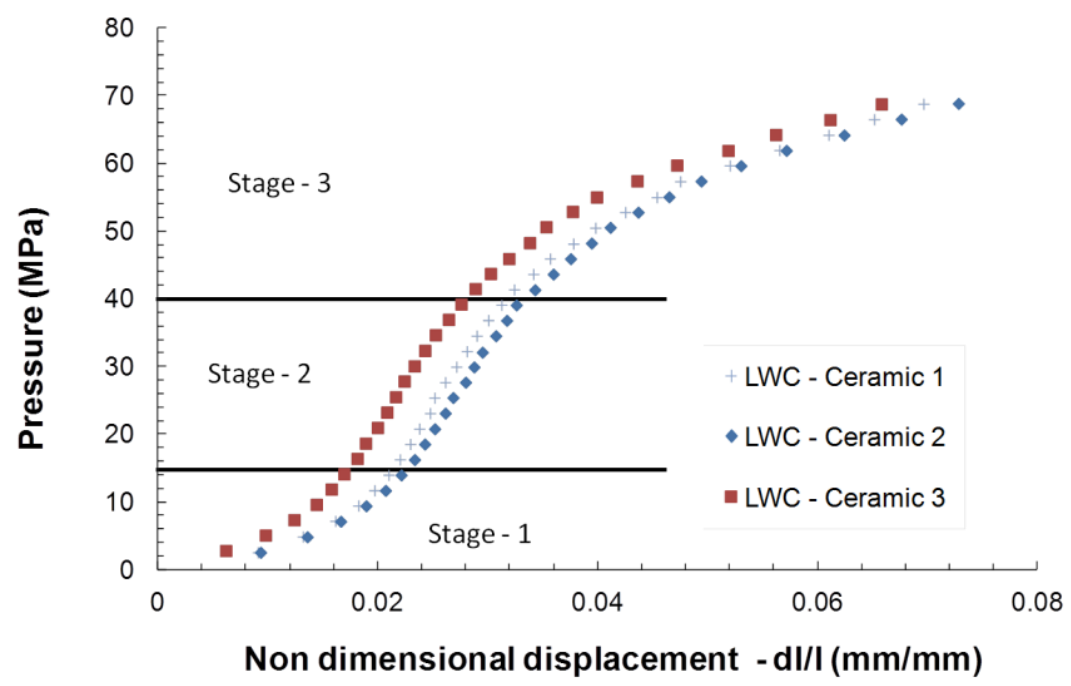

Fig. 4.10. Pressure vs non dimensional displacement LWC ceramic baseline 
Table. 4.2. Crush and Stiffness data for walnut ceramic mixture

\begin{tabular}{|c|c|c|c|c|}
\hline & \multicolumn{3}{|c|}{ Walnut + Ceramic } \\
& \multicolumn{2}{|c|}{ Weight percent crush (\%) } & \multicolumn{2}{c|}{ Effective Stiffness (MPa) } \\
\hline $\begin{array}{c}\text { Deformable } \\
\text { particles by } \\
\text { weight (\%) }\end{array}$ & Average & Std dev & Average & Std dev \\
\hline 6 & 18.05 & 0.17 & 1050.06 & 91.78 \\
\hline 10 & 16.23 & 0.68 & 792.20 & 45.98 \\
\hline 18 & 12.77 & 0.64 & 591.99 & 46.79 \\
\hline 25 & 9.51 & 0.23 & 526.66 & 6.91 \\
\hline 33 & 6.79 & 0.22 & 499.93 & 7.86 \\
\hline
\end{tabular}

Table. 4.3. Crush and Stiffness data for Al needles ceramic mixture

\begin{tabular}{|c|c|c|c|c|}
\hline \multicolumn{5}{|c|}{ Al Needles + Ceramic } \\
\hline & \multicolumn{2}{|c|}{$\begin{array}{c}\text { Weight percent crush } \\
(\%)\end{array}$} & \multicolumn{2}{c|}{ Effective Stiffness (MPa) } \\
\hline $\begin{array}{c}\text { Deformable } \\
\text { particles by } \\
\text { weight (\%) }\end{array}$ & Average & Std dev & Average & Std dev \\
\hline 6 & 14.42 & 1.54 & 1344.42 & 108.87 \\
\hline 10 & 16.29 & 0.30 & 894.51 & 36.76 \\
\hline 18 & 13.57 & 0.87 & 595.39 & 51.65 \\
\hline 25 & 12.01 & 0.31 & 439.38 & 5.11 \\
\hline 33 & 9.26 & 0.38 & 365.39 & 14.00 \\
\hline
\end{tabular}


Table. 4.4. Crush and Stiffness data for Al pellets ceramic mixture

\begin{tabular}{|c|c|c|c|c|}
\hline \multicolumn{5}{|c|}{ Al Pellets + Ceramic } \\
& \multicolumn{2}{|c|}{ Weight percent crush (\%) } & \multicolumn{2}{c|}{ Effective Stiffness (MPa) } \\
\hline $\begin{array}{l}\text { Deformable } \\
\text { particles by } \\
\text { weight (\%) }\end{array}$ & Average & Std dev & Average & Std dev \\
\hline 6 & 13.11 & 0.28 & 2056.83 & 127.69 \\
\hline 10 & 11.91 & 0.69 & 1865.20 & 63.24 \\
\hline 18 & 10.53 & 0.32 & 1657.60 & 75.67 \\
\hline 25 & 8.60 & 0.23 & 1434.67 & 46.63 \\
\hline 33 & 5.29 & 0.22 & 1353.07 & 73.57 \\
\hline
\end{tabular}

Table. 4.5. Crush and Stiffness data for pistachio ceramic mixture

\begin{tabular}{|c|c|c|c|c|}
\hline & \multicolumn{3}{|c|}{ Pistachio + Ceramic } \\
\hline & Weight percent crush (\%) & \multicolumn{2}{c|}{ Effective Stiffness (MPa) } \\
\hline $\begin{array}{c}\text { Deformable } \\
\text { particles by } \\
\text { weight (\%) }\end{array}$ & Average & Std dev & Average & Std dev \\
\hline 6 & 14.44 & 0.71 & 1239.97 & 49.41 \\
\hline 10 & 11.14 & 0.75 & 1079.00 & 75.82 \\
\hline 18 & 11.14 & 0.53 & 651.47 & 6.42 \\
\hline 25 & 8.53 & 0.12 & 600.69 & 17.54 \\
\hline 33 & 6.89 & 0.54 & 434.95 & 6.95 \\
\hline
\end{tabular}


Fig. 4.11a and $4.11 \mathrm{~b}$ show the variation of average crush and effective pack stiffness for the ceramic - walnut mixtures. The vertical black lines on each data point in the figures represent the variability in the data for each composition which is found to be small. Fig. 4.11c shows the pressure vs displacement data for the walnut - ceramic mixture, the curve on the left represents the baseline ceramic response. It is observed that stiffness reduces with the addition of deformable particles. From Fig. 4.11a it is also observed that for small concentrations of softer particles in particular $6 \%$ and $10 \%$ the crush is actually higher compared to the baseline ceramic. This can be attributed to the disruption of the packing arrangement. The softer walnut particles are larger compared to the ceramic particles and also have a low bulk density of $0.76 \mathrm{~g} / \mathrm{cm}^{3}$ compared to $1.56 \mathrm{~g} / \mathrm{cm}^{3}$ for the LWC ceramic which results in an increase in the initial pack porosity and reduce the number of ceramic particles surrounding other ceramics thus reducing the co-ordination number. Ceramic is a brittle material and particles fracture by breaking along the diameter when the maximum principle stress in tension exceeds the fracture strength. A higher co-ordination number of ceramic particles prevent the tensile stresses from growing and keep the particles predominantly in compression. A decrease in co-ordination number results in a larger number of particles getting subjected to tensile stresses and strains resulting in their fracturing. At higher concentrations the softer particles provide a cushioning to the harder ceramic particles and absorb the applied load by undergoing greater deformation and preventing particle fracture. This also explains the observation that with the addition of softer particles the stiffness initially reduces significantly but later at higher concentration it plateaus as the loss in stiffness due to the addition of softer particles is compensated by the reduction in ceramic particle fracture which also causes a loss in stiffness. Notice that in Fig. 10 the curve shows a very distinct " $\mathrm{S}$ " shape, with a pronounced change in slope due to particle fragmentation. At higher concentrations this change becomes more subtle. 


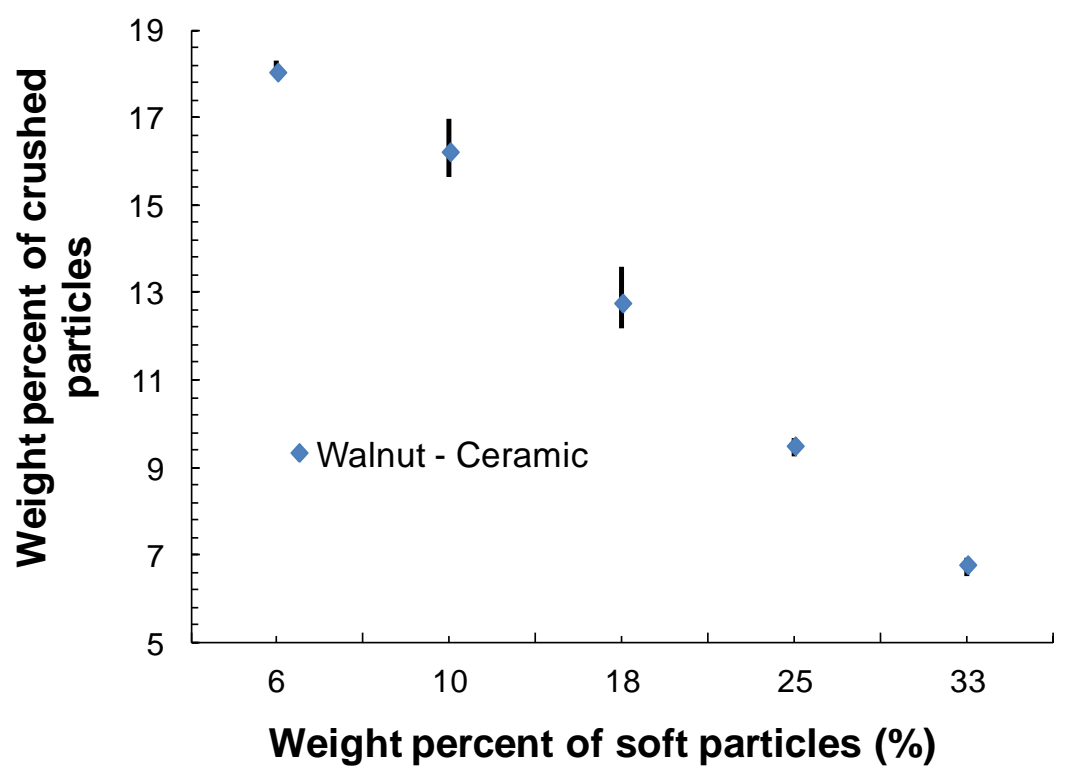

(a)

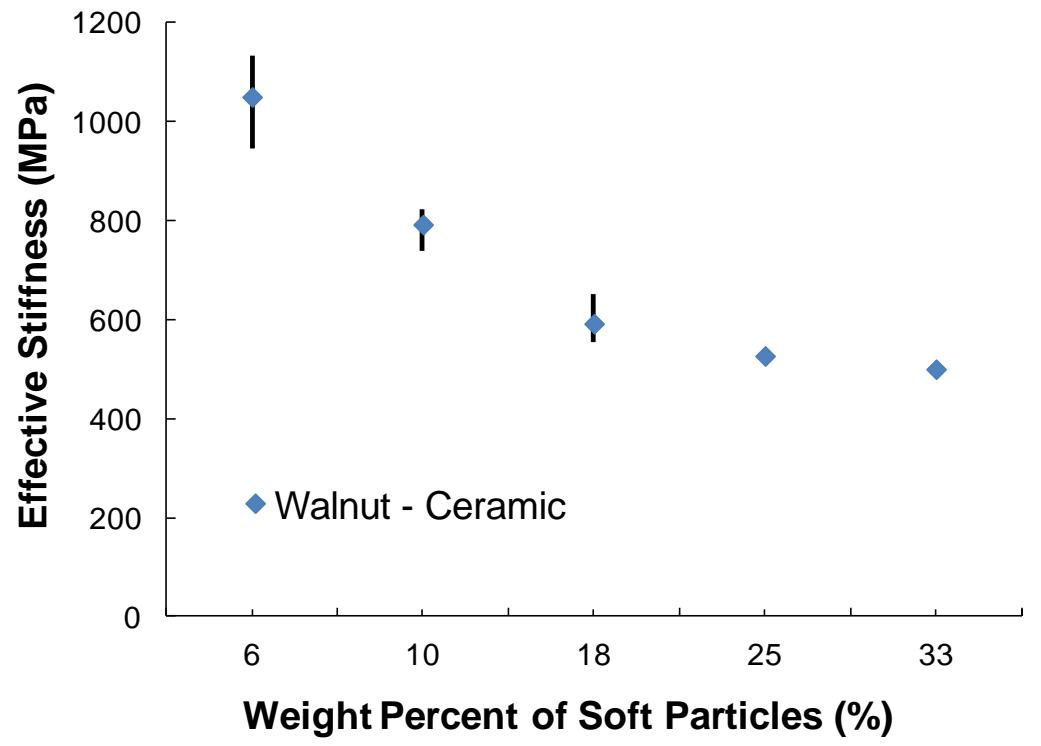

(b)

Fig. 4.11. Walnut-Ceramic mixture (a) Crush as a function of weight percent of soft particles (b) Effective stiffness as a function of weight percent of soft particles and (c) Pressure vs non dimensional displacement 


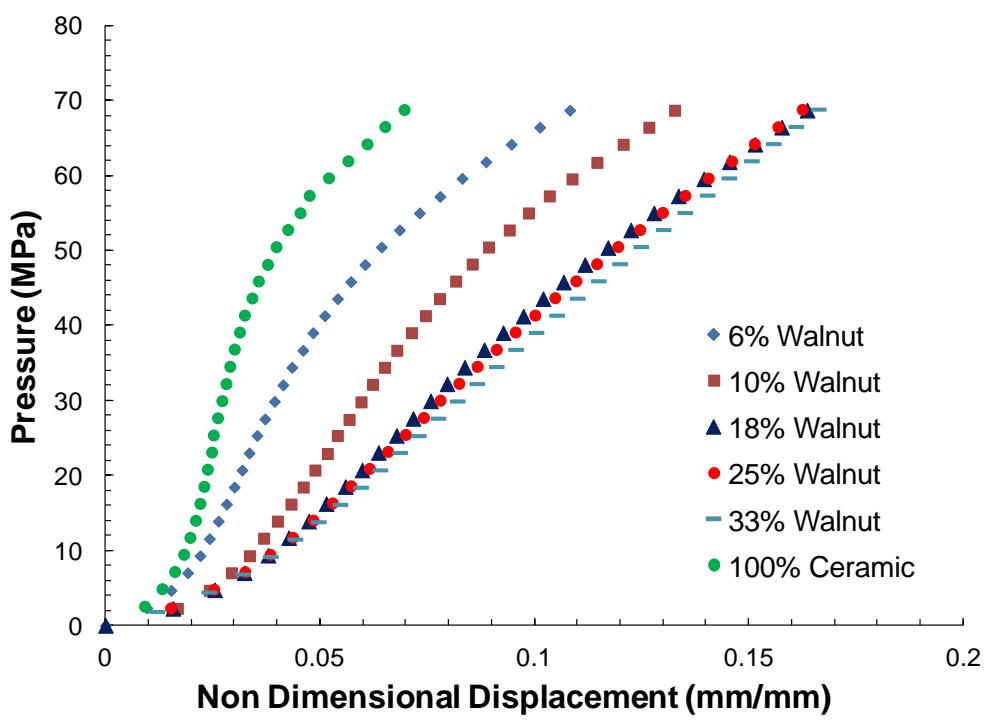

(c)

Fig. 4.11. Continued

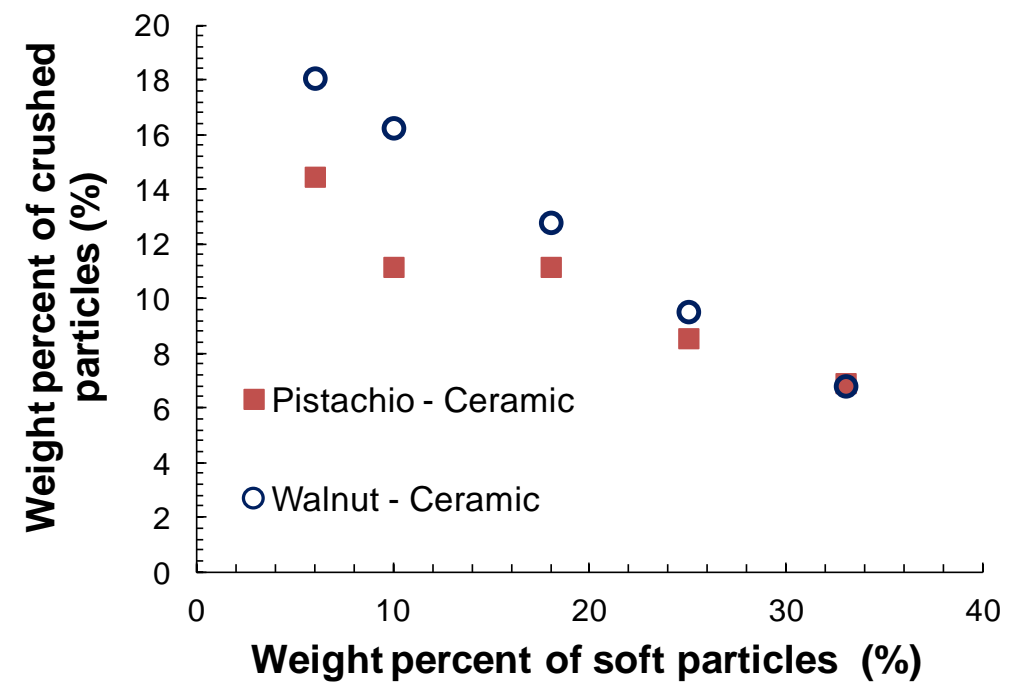

(a)

Fig. 4.12. Walnut-Ceramic and Pistachio Ceramic mixtures (a) Average percent crushes (b) Average stiffness (c) Walnut - Ceramic mixture image and (d) Pistachio - Ceramic mixture image 


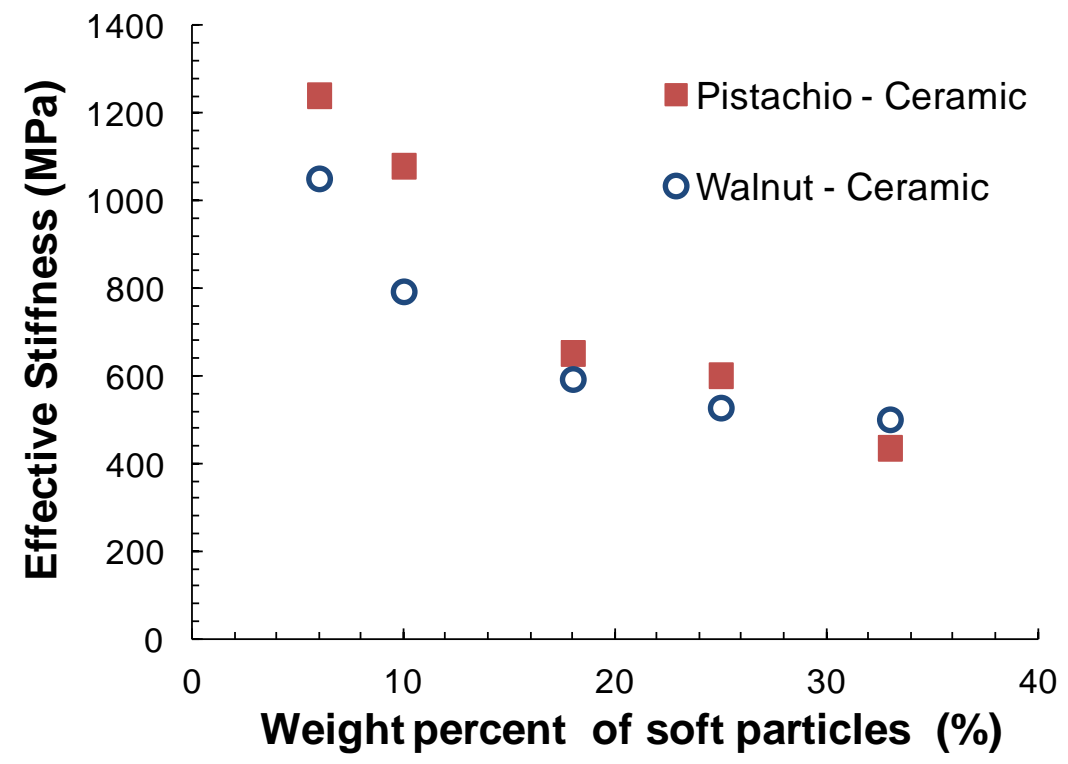

(b)

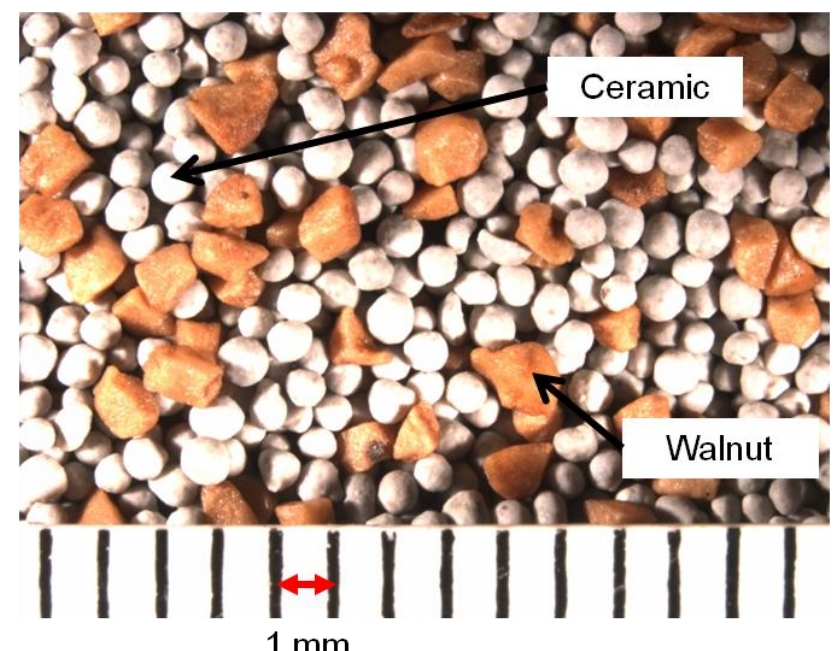

(c)

Fig. 4.12. Continued 


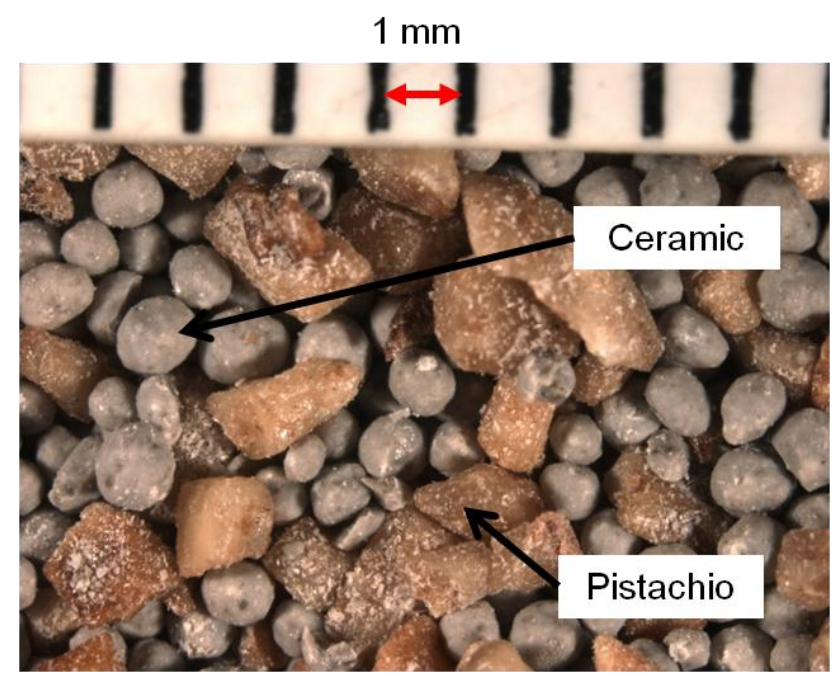

(d)

Fig. 4.12. Continued

A comparison of average crush and stiffness variation with composition for walnut - ceramic and pistachio - ceramic mixtures is presented in Fig. 4.12a and 4.12b. It is found that the variation of crush and stiffness with composition is similar for the two mixtures with pistachios giving better results. This can be attributed to a more uniform particle shape distribution for pistachios and a stiffer response to compression compared to walnut. The two different mixtures are shown in Fig. 4.12c and 4.12d.

Similarly comparing the average crush (Fig. 4.13a) and average stiffness (Fig. 4.13b) variation with composition for $\mathrm{Al}$ needles - ceramic and walnut - ceramic mixtures it is observed that the crush for $\mathrm{Al}$ needles at $6 \%$ is lower than the walnut mixture and then it increases and stays higher for the higher mixture concentrations. This can be attributed to the lower bulk density of walnut compared to Al needles. At $6 \%$ mixture concentration the number of $\mathrm{Al}$ needles is not significantly high enough to disrupt the packing and hence the number of particles fracturing is not as high as in case of walnut. At higher concentrations the shape of the Al needles which has a high aspect ratio (Fig. 4.13c) starts to play a significant role in disrupting the packing and thus 
increases the percentage crush in comparison to the walnut mixture. It is also observed that the crush is higher than the baseline crush for all the concentrations except $33 \%$ for the $\mathrm{Al}$ needles mixture on the other hand for the walnut-ceramic and pistachio-ceramic mixtures the crush is equal to or less then the baseline at concentrations of $18 \%$ or higher. In the same way we observe that for the $6 \%$ and $10 \%$ mixtures the $\mathrm{Al}$ needles shows a higher stiffness compared to walnut mixture which can be attributed to the higher strength and elastic modulus of pure Al compared to walnut. At higher concentrations the higher crush observed in Al needle - ceramic mixture results in stiffness reduction which eventually reduces below that of the walnut - ceramic mixture for the $25 \%$ and $33 \%$ mixtures.

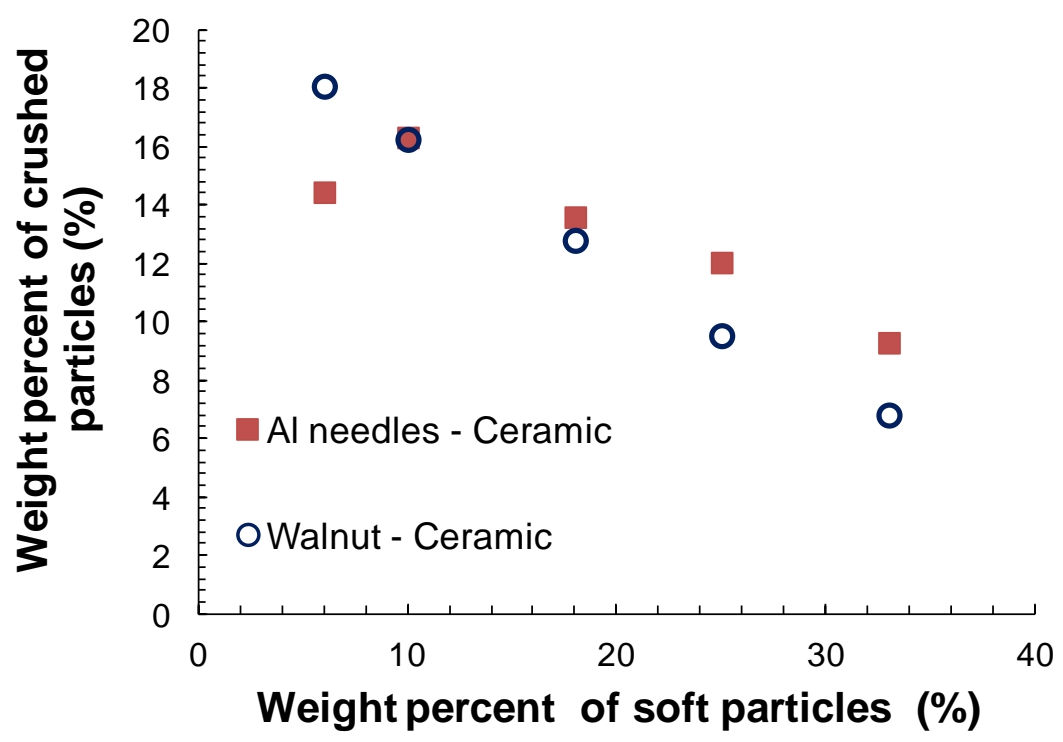

(a)

Fig. 4.13. Walnut-Ceramic and Al needles-Ceramic mixture (a) Average percent crushes (b) Average effective stiffness and (c) Al needles - ceramic mixture image 


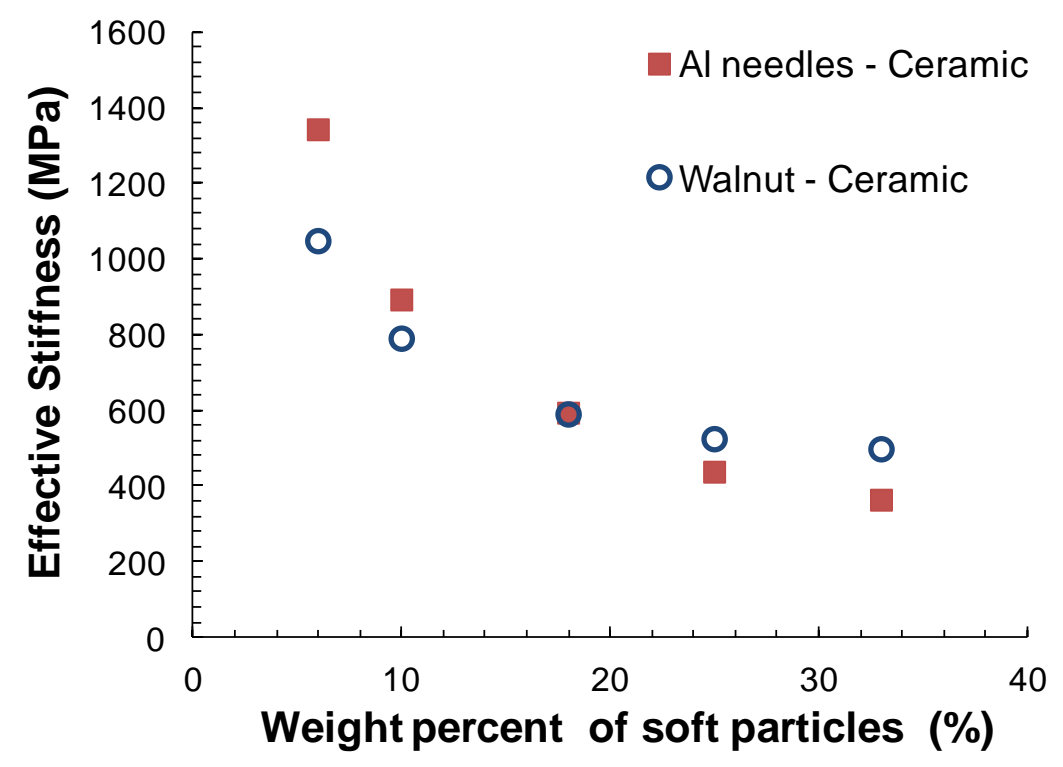

(b)

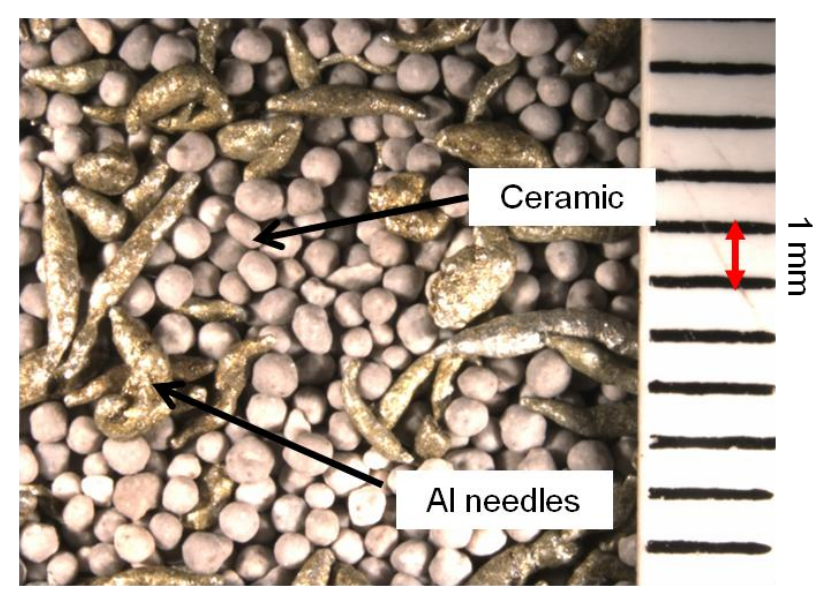

(c)

Fig. 4.13. Continued

Comparing Al needles - ceramic and Al pellets - ceramic crush and stiffness response (Fig 4.14a and 4.14b) it is observed that the crush for Al pellets - ceramic mixture is much lower than all the other mixtures considered. This can be attributed to the higher bulk density of Al pellets due to their highly uniform spherical shape (Fig 
4.14c) compared to $\mathrm{Al}$ needles resulting in little pack disruption and hence lower crush. As in case of the other mixtures crush reduces with increase in concentration of the soft particles, also note that for $\mathrm{Al}$ pellets apart from the $6 \%$ mixture the crush is lower than the baseline for all the other concentrations. It is also noticed that the Al pellet - ceramic mixture has a much higher stiffness compared to the other mixtures and this is also attributed to the higher bulk density which results in less pack disruption and low crush.

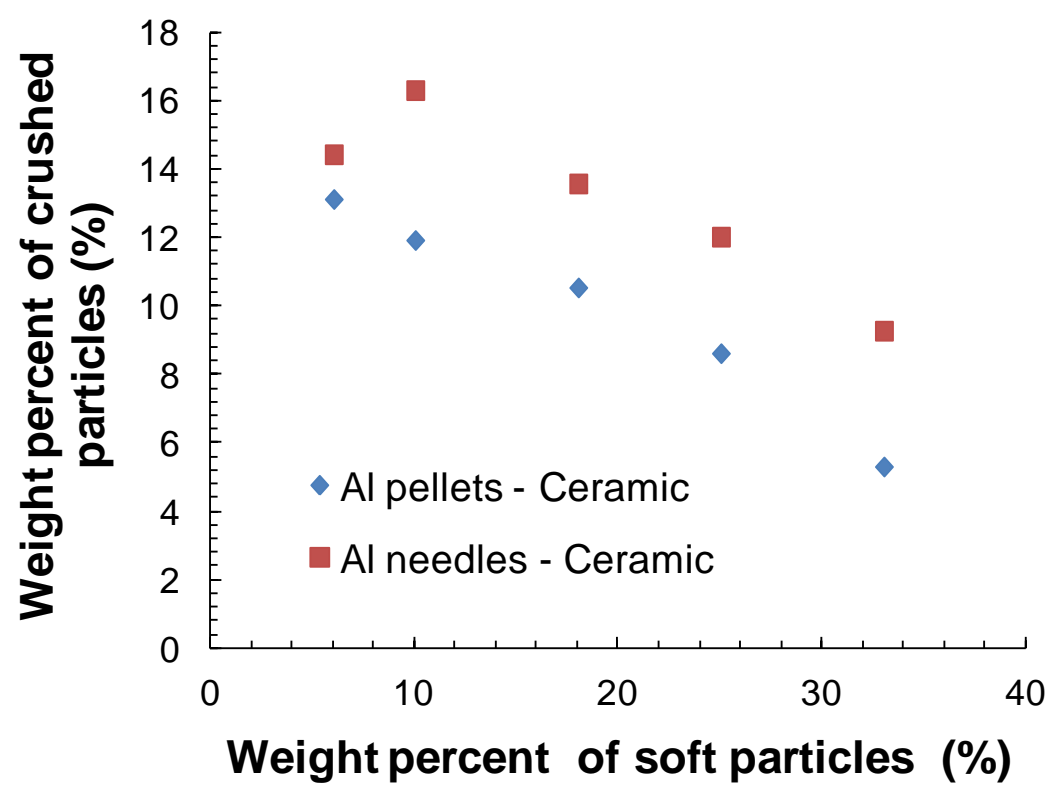

(a)

Fig. 4.14. Al pellets - Ceramic and Al needles - Ceramic mixtures (a) Average percent crushes (b) Average effective stiffness and (c) Al pellets - ceramic mixture image 


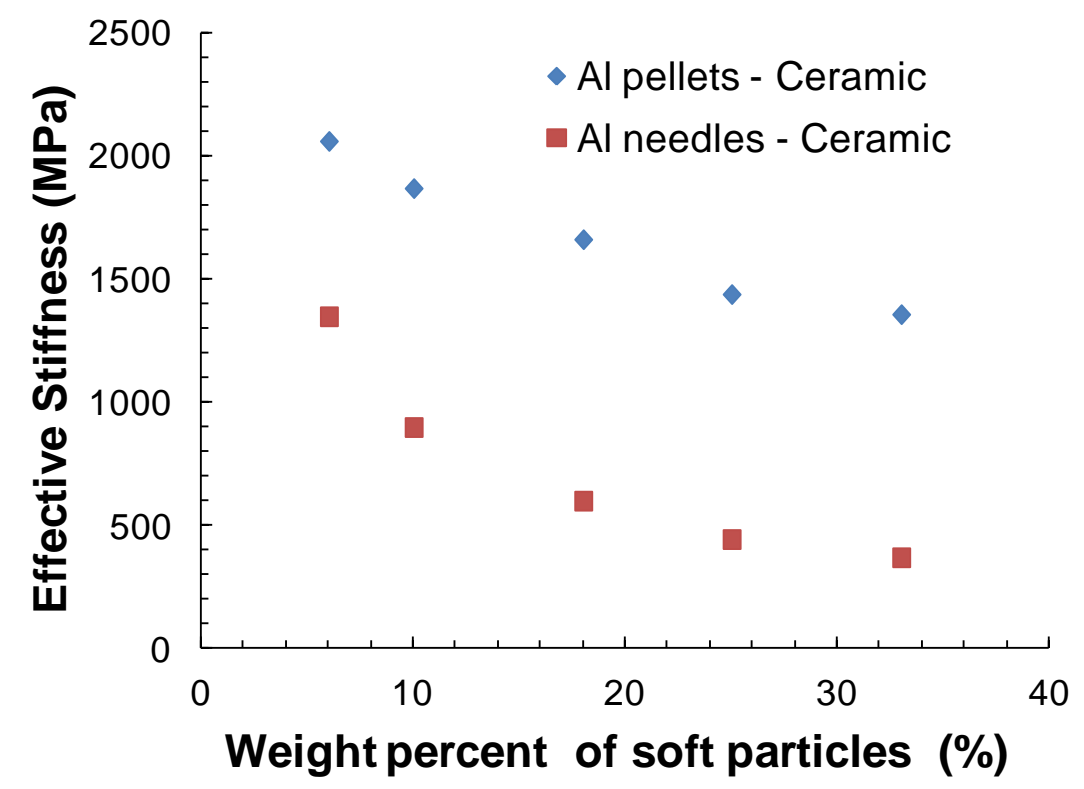

(b)

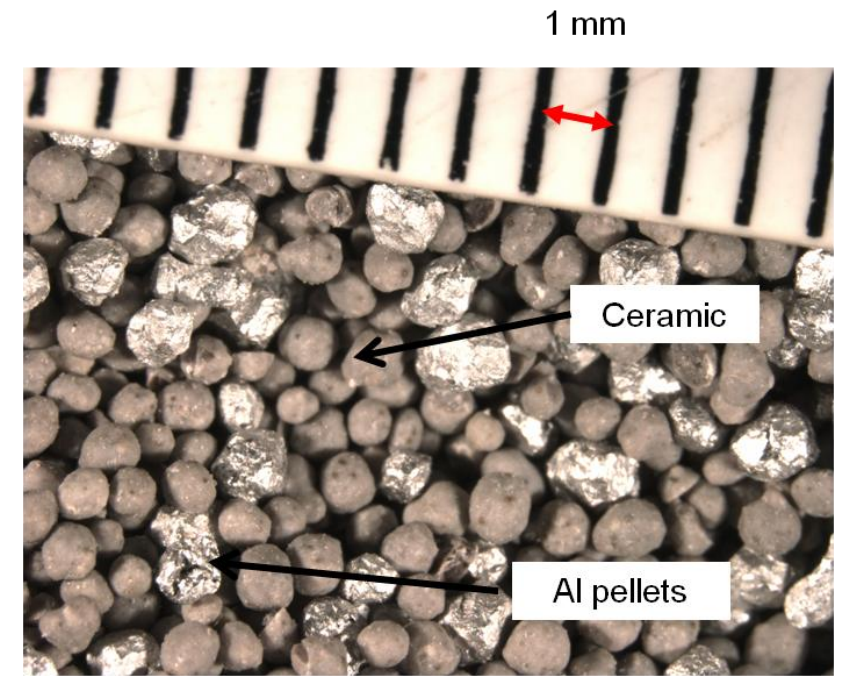

(c)

Fig. 4.14. Continued 


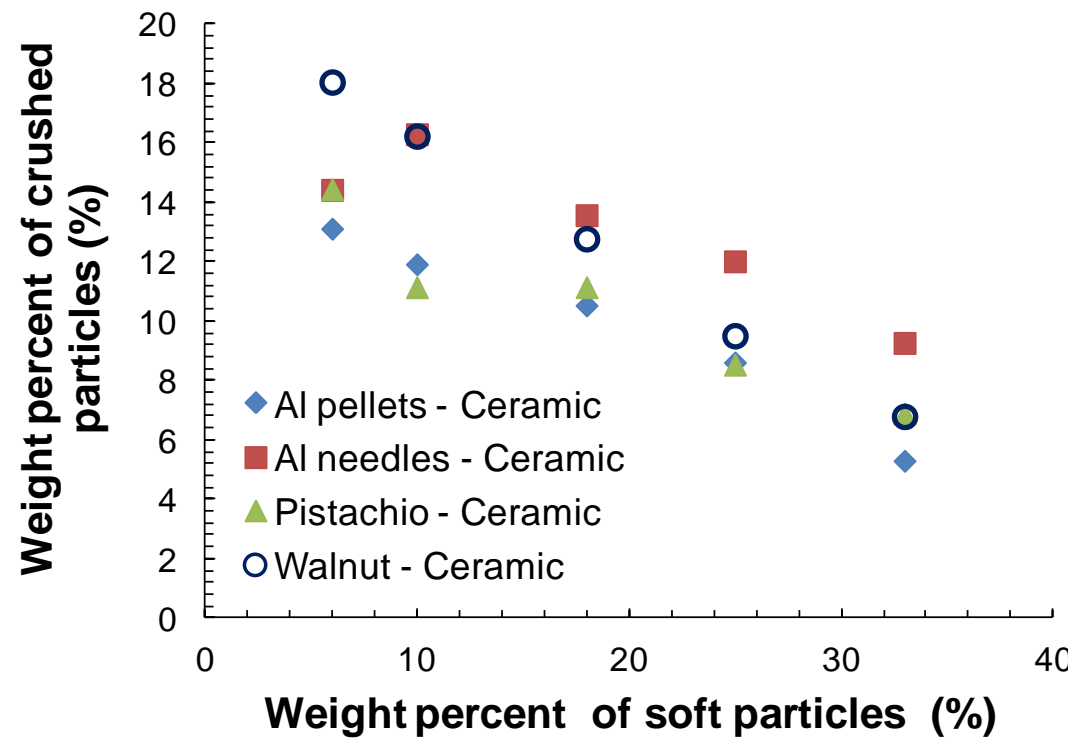

(a)

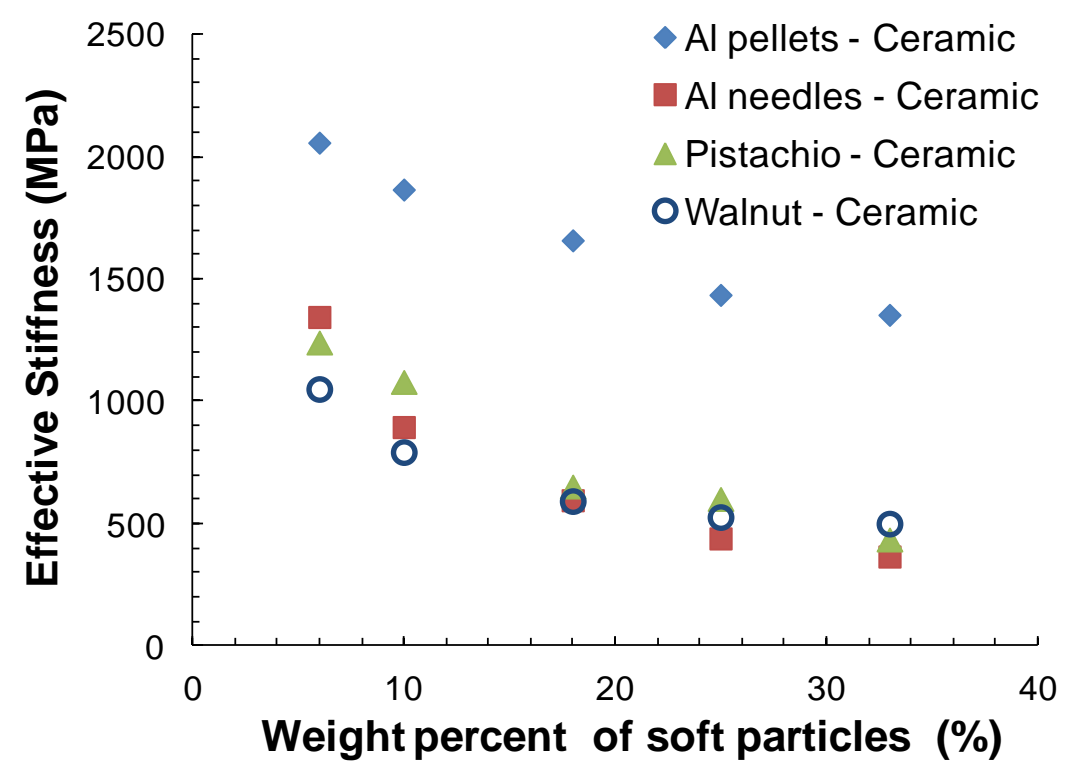

(b)

Fig. 4.15. (a) Comparisons of average crush for all mixtures (b) Comparison of average stiffness for all mixtures 
The variation of average crush and average stiffness for all the mixtures is compared in Fig. 4.15a and 4.15b respectively. As stated previously the Al pellets ceramic mixture gives the best response at all the mixtures for the stiffness. For crush it is observed that pistachio - ceramic mixture and Al pellets - ceramic mixtures both give a lower crush compared to the other two mixtures. The Al needles - ceramic mixture gives the highest crush for all concentrations except for $6 \%$ where the highest crush is observed for the walnut - ceramic mixtures.

It can be stated that the Al pellets - ceramic is the ideal mixture to be employed in the hydraulic fractures from a point of view of crush resistance and pack stiffness. Also the pistachio - ceramic mixture provides good results for crush resistance but the stiffness response is similar to the walnut - ceramic response. It needs to be stated that crush resistance and pack stiffness is only one part of proppant characterization and the final acceptability of a mixture is dependent on its effectiveness of maintaining high pack porosity and preventing flowback which needs to be investigated.

\subsubsection{Results and Discussion: HSP Mixtures}

For the baseline tests on HSP sintered bauxite proppants the average percentage crush at the applied pressure of $103 \mathrm{MPa}$ or $15000 \mathrm{psi}$ (weight of fractured particles with size less than 400 microns related to the original sample weight of $50.0 \mathrm{~g}$ ) is obtained as $6.03 \%$ with a maximum of $6.37 \%$ and minimum of $5.48 \%$ with a standard deviation of $0.48 \%$. It can be clearly observed that the HSP proppant pack has a much lower crush compared to the LWC pack even at a much higher pressure which is consistent with its higher stiffness and strength. Also the crush is less than $10 \%$ which means the pack is safe to employ at the 15000 psi closure stress. The average effective stiffness for the baseline tests is calculated to be $7943.46 \mathrm{MPa}$ with a standard deviation of $421.74 \mathrm{MPa}$, the minimum and maximum values are calculated as $7459.9 \mathrm{MPa}$ and $8293.8 \mathrm{MPa}$ respectively. The pack stiffness values are also significantly higher than those of the 
LWC proppants. Fig. 4.16 shows the load vs displacement response for the HSP baseline and the $10 \%$ and $25 \% \mathrm{Al}$ needles mixture. The trends here are similar to those observed with the ISP proppant mixtures with the stiffness reducing with the addition of softer particles.

For the HSP Al needles mixture, Table. 4.6 presents the average and standard deviation of percent crush and pack stiffness. A significant reduction in effective pack stiffness is reported with the addition of softer particles compared to the baseline. Observations for crush show that the percent crush is still lower than $10 \%$ which is the safe limit according to the API RP 60 standards but at $9.46 \%$ and $7.38 \%$ is higher than the baseline crush. Again, similar to the LWC mixtures at $25 \%$ mixture strength crush is reduced compared to the $10 \%$ mixture but here it is still higher than the baseline. Table. 4.7 presents the average and standard deviation data for the HSP and Al sphere/pellets mixture. We again observe a significant decline in the effective pack stiffness. The decline is not as large as observed with the Al needles. The percentage crush is also smaller than the baseline, this is similar to what is observed for the LWC mixtures and can be attributed to the shape difference. A comparison of average crush and average stiffness of the LWC and the HSP mixtures is presented in Table. 4.8.

Table. 4.6. Crush and Stiffness data for HSP with Al needle mixture

\begin{tabular}{|c|c|c|c|c|}
\hline \multicolumn{5}{|c|}{ Al Needles + HSP } \\
\hline & \multicolumn{2}{|c|}{ Weight percent crush (\%) } & \multicolumn{2}{l|}{ Effective Stiffness (MPa) } \\
\hline $\begin{array}{c}\text { Deformable } \\
\text { particles by } \\
\text { weight (\%) }\end{array}$ & Average & Std dev & Average & Std dev \\
\hline 10 & 9.46 & 0.17 & 1414.90 & 57.00 \\
\hline 25 & 7.38 & 0.12 & 531.29 & 5.21 \\
\hline
\end{tabular}




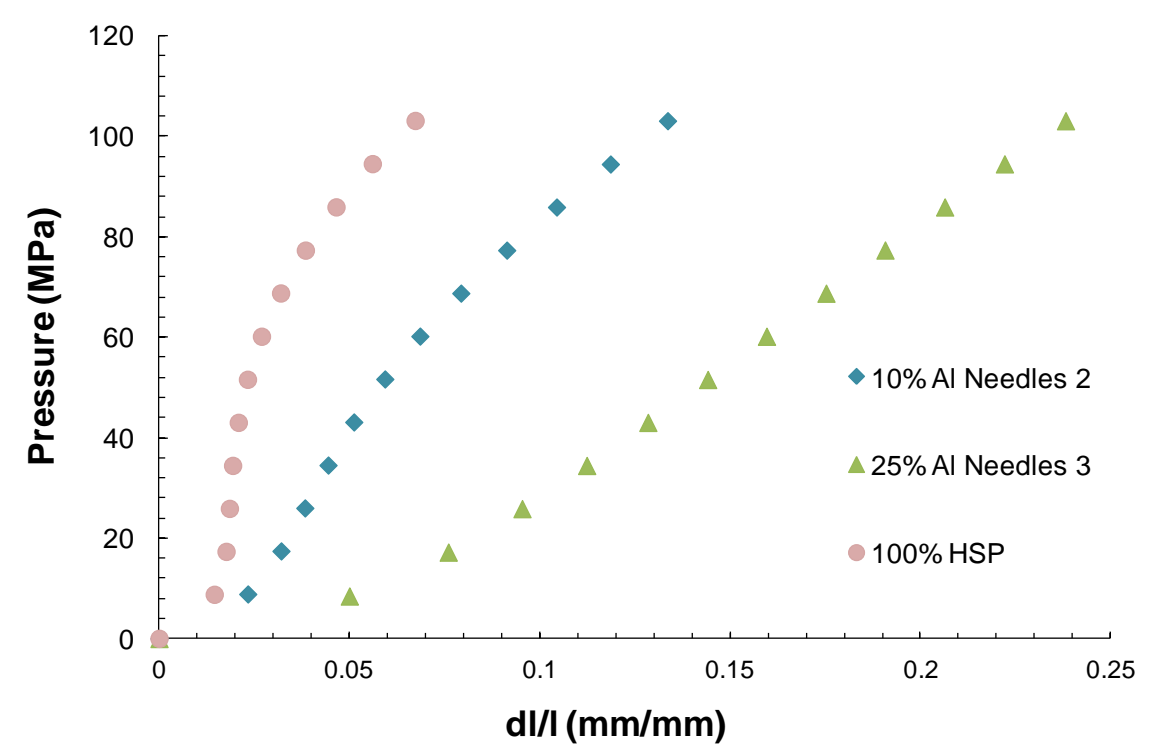

Fig. 4.16. Pressure vs non dimensional displacement Al Needles - HSP mixture

Table. 4.7. Crush and stiffness data for HSP with Al sphere mixture

\begin{tabular}{|c|c|c|c|c|}
\hline \multicolumn{5}{|c|}{ Al Spheres + HSP } \\
\hline & \multicolumn{2}{|c|}{ Weight percent crush (\%) } & \multicolumn{2}{|c|}{ Effective Stiffness (MPa) } \\
\hline $\begin{array}{c}\text { Deformable } \\
\text { particles by } \\
\text { weight (\%) }\end{array}$ & Average & Std dev & Average & Std dev \\
\hline 10 & 5.56 & 0.36 & 4250.90 & 371.00 \\
\hline 25 & 4.51 & 0.12 & 2044.20 & 99.88 \\
\hline
\end{tabular}


Table. 4.8. Average crush and stiffness comparison of Al needles and Al spheres mixtures with LWC and HSP proppants

\begin{tabular}{|c|c|c|c|c|}
\hline & \multicolumn{2}{|c|}{ LWC (ceramic) } & \multicolumn{2}{c|}{ HSP (bauxite) } \\
\hline & Crush (\%) & $\begin{array}{c}\text { Stiffness } \\
(\mathrm{MPa})\end{array}$ & Crush (\%) & $\begin{array}{c}\text { Stiffness } \\
(\mathrm{MPa})\end{array}$ \\
\hline Baseline & 11.9 & 2408 & 6.03 & 7913.46 \\
\hline $10 \% \mathrm{Al}$ needles & 16.29 & 894.51 & 9.47 & 1414.90 \\
\hline $10 \% \mathrm{Al}$ spheres & 11.14 & 1865.2 & 5.57 & 4250.90 \\
\hline $25 \% \mathrm{Al}$ needles & 12.01 & 439.38 & 7.38 & 531.29 \\
\hline $25 \% \mathrm{Al}$ spheres & 8.60 & 1434.67 & 4.52 & 2044.20 \\
\hline \multicolumn{5}{|c}{} \\
\hline NOTE: Crush for HSP at 15000 psi or 103 MPa, for LWC crush at 10000 \\
psi or 68 MPa
\end{tabular}

In both cases for LWC as well as HSP addition of $10 \% \mathrm{Al}$ needles results in increase of percent crush, at the same time a steep decline in pack stiffness is observed. In particular the decline is much steeper with the HSP particles. Even with Al spheres we observe a significant decline in pack stiffness for the HSP particles while crush remains practically unchanged. Comparing LWC and HSP it can be argued that addition of $\mathrm{Al}$ needles or spheres is probably more detrimental to the HSP pack response.

\subsubsection{Results and Discussion: Ottawa Sand Mixtures}

Fig. 4.17 shows the load vs displacement response for the sand baseline and the $10 \%$ walnut and $10 \%$ pistachio mixtures. The trends here are similar to those observed with the LWC proppant mixtures with the stiffness reducing with the addition of softer particles. Again as observed previously pistachio mixtures tend to be slightly stiffer compared to the walnut mixtures. The average stiffness and percent crush for the three 
sets of sand mixtures and a comparison with the corresponding LWC mixtures is provided in Table. 4.9.

At the applied 7500 psi pressure sand shows significant crush average $33.84 \%$. Addition of $10 \%$ walnut or pistachio results in the reduction of percent crush to $\sim 25 \%$. This value is still significantly larger than the limit of $10 \%$. Similarly effective pack stiffness is reduced with the addition of softer particles but the decline is not as steep as the LWC mixtures. If we compare the load vs displacement response for the baseline materials in Fig. 4.11c, 4.16 and 4.17 it is observed that for the three materials softening which indicates onset of significant crushing of the particles begins at $40 \mathrm{MPa}$ for LWC, $60 \mathrm{MPa}$ for HSP and $25 \mathrm{MPa}$ for sand. This is directly attributed to the strength of the base material.

Comparing the mixtures for the three base materials it is observed that for the economically feasible range of $10 \%$ soft particles improvement in crush resistance is obtained only for sand. For the LWC and HSP addition of small percent of softer particles (in particular Al needles) increases particle fragmentation while significantly decreasing the effective pack stiffness. Again as stated previously the final applicability of a mixture rests with its economic feasibility and capability of enhancing flowback resistance while maintaining sufficient pack permeability.

Table. 4.9. Average crush and stiffness of baseline sand and 10\% walnut and pistachio mixtures, also compared with LWC mixtures

\begin{tabular}{|l|c|c|c|c|c|c|}
\hline & \multicolumn{2}{|c|}{ Baseline } & \multicolumn{2}{c|}{$\mathbf{1 0 \%}$ Walnut } & \multicolumn{2}{c|}{$\mathbf{1 0 \%}$ Pistachio } \\
\hline & $\begin{array}{c}\text { Crush } \\
(\%)\end{array}$ & $\begin{array}{c}\text { Stiffness } \\
(\mathrm{MPa})\end{array}$ & $\begin{array}{c}\text { Crush } \\
(\%)\end{array}$ & $\begin{array}{c}\text { Stiffness } \\
(\mathrm{MPa})\end{array}$ & $\begin{array}{c}\text { Crush } \\
(\%)\end{array}$ & $\begin{array}{c}\text { Stiffness } \\
(\mathrm{MPa})\end{array}$ \\
\hline $\begin{array}{l}\text { LWC } \\
\text { (ceramic) }\end{array}$ & 11.9 & 2408 & 16.23 & 792.2 & 11.14 & 1079 \\
\hline Sand & 33.84 & 1161.35 & 25.64 & 638.54 & 25.55 & 669.17 \\
\hline
\end{tabular}

NOTE: Crush for Sand at 7500 psi or $51 \mathrm{MPa}$, for LWC crush at 10000 psi or $68 \mathrm{MPa}$ 


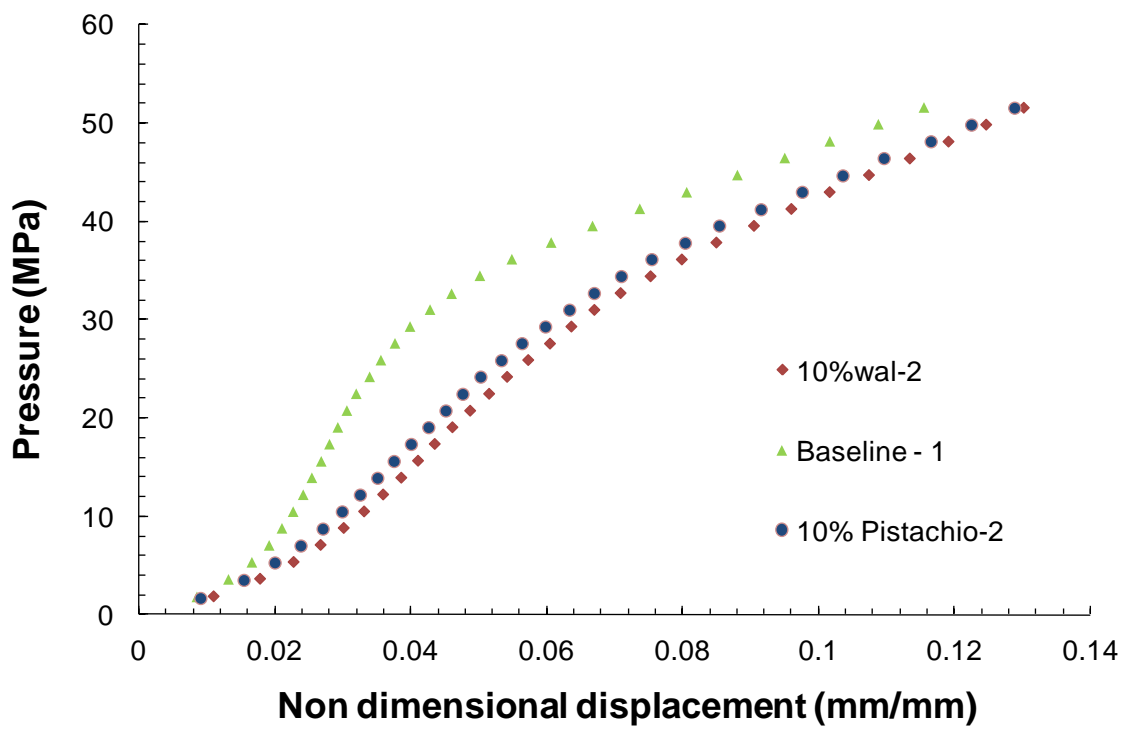

Fig. 4.17. Pressure vs non dimensional displacement baseline, 10\% walnut and 10\% pistachio - Ottawa sand mixture 


\section{COMPARISON OF EXPERIMENTS AND MODELS: CONFINED COMPRESSION}

In this section polydisperse FEM models for confined compression are compared with the crush test results. Two different models, ie with damage and with fracture form the basis in these observations of pressure vs non-dimensional displacement response and of particle crush. The models are also compared to each other from the point of view of void space available for fluid flow. Finally, the effect of particle shape and size on the pack response is presented by comparing three $25 \%$ pure Al needles - ceramic models with small needles, large needles and spheres.

\subsection{Models with Ceramic Damage}

The pressure vs displacement responses for the four Type-A (walnut - ceramic) models (Fig. 3.12, section 3.2) are compared to the corresponding crush test responses for LWC mixtures in Fig. 5.1. It is observed that apart from the $6 \%$ mixture the rest of the models show a stiffer response than the test data. This is primarily due to the absence of particle fragmentation in the models. In the tests we observe a sudden change in slope of the curves with the onset of particle fracture. This also causes additional particle rearrangement to take place which causes a further change in slope. Because we are not simulating particle fracture we only obtain the continuously rising curves with increasing stiffness and no sudden change in the slope is observed. Thus to better simulate the crush test response it is imperative to incorporate particle fracture which is discussed next. 


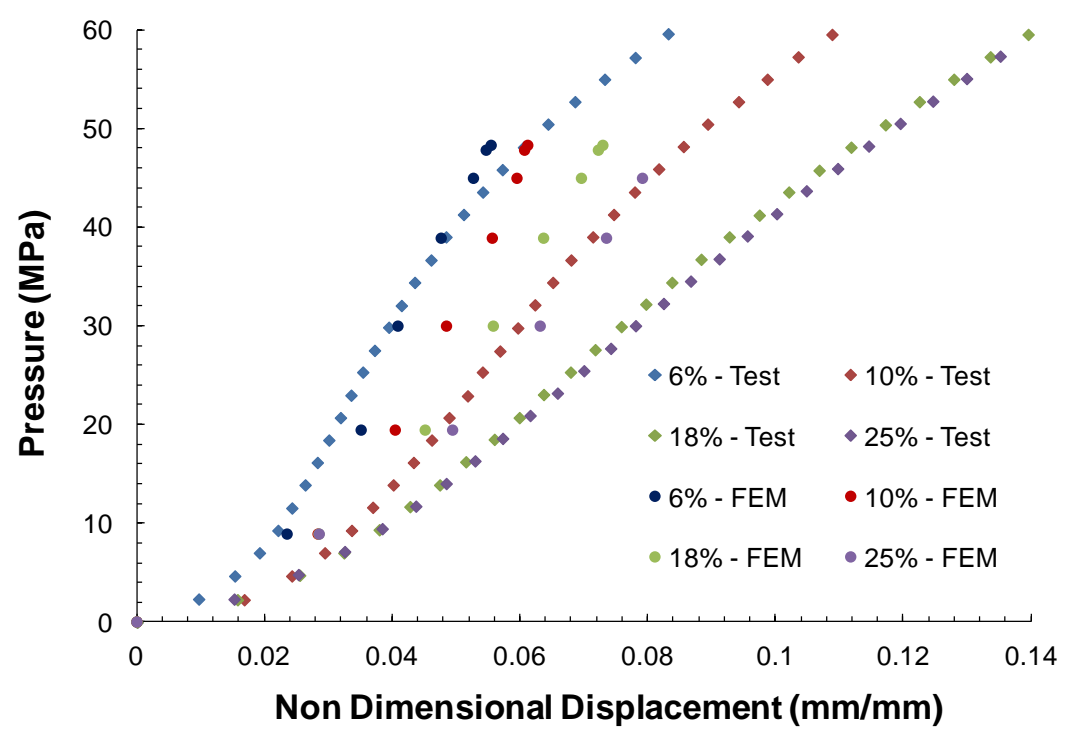

Fig. 5.1. Pressure vs non-dimensional displacement comparison of Type-A models with damage and confine compression/crush tests

\subsection{Models with Ceramic Fracture $-10 \%$ and $25 \%$ Walnut}

The pressure vs non-dimensional displacement plots for the $10 \%$ and $25 \%$ walnut - LWC ceramic mixture are presented in Fig. 5.2. Computational models incorporated element deletion algorithm to simulate fracture in the ceramic particles. In these models the ceramic specific gravity is 2.6 compared to 3.6 for models discussed in section 3 . The initial configuration of the two models prior to loading is displayed in Fig. 5.3a and 5.3b. It can be observed that at lower pressures, the model and test responses match closely. At higher pressures, the models tend to diverge from the test response. In contrast to the damage models where the divergence is represented as a stiffer response, the present fracture models present a softer response. This can be attributed directly to i) weaker material response from the ceramic particles - the failure strain at $0.8 \%$ is equivalent to $28 \mathrm{~N} / \mathrm{m}$ fracture energy which may be less than the actual value for the ISP ceramic particle and ii) element deletion that introduces spurious weakness in the model 
as a deleted element is incapable of carrying load in compression. Moreover the particle pack here is modeled with only 400 particles, as the elements are deleted the number of ceramic particles which carry load is reduced. With a larger model this reduction would not significantly alter the total number of particles and hence the load carrying capacity of the pack. In case of the tests even when a large number of particles are fractured the total number of particles is large enough to carry the load. Hence the softening is more gradual for the tests while it is more sudden for the models. Furthermore, the models are simulated under 2D plane strain conditions rather than the actual $3 \mathrm{D}$ for the test which may result in a weaker response.

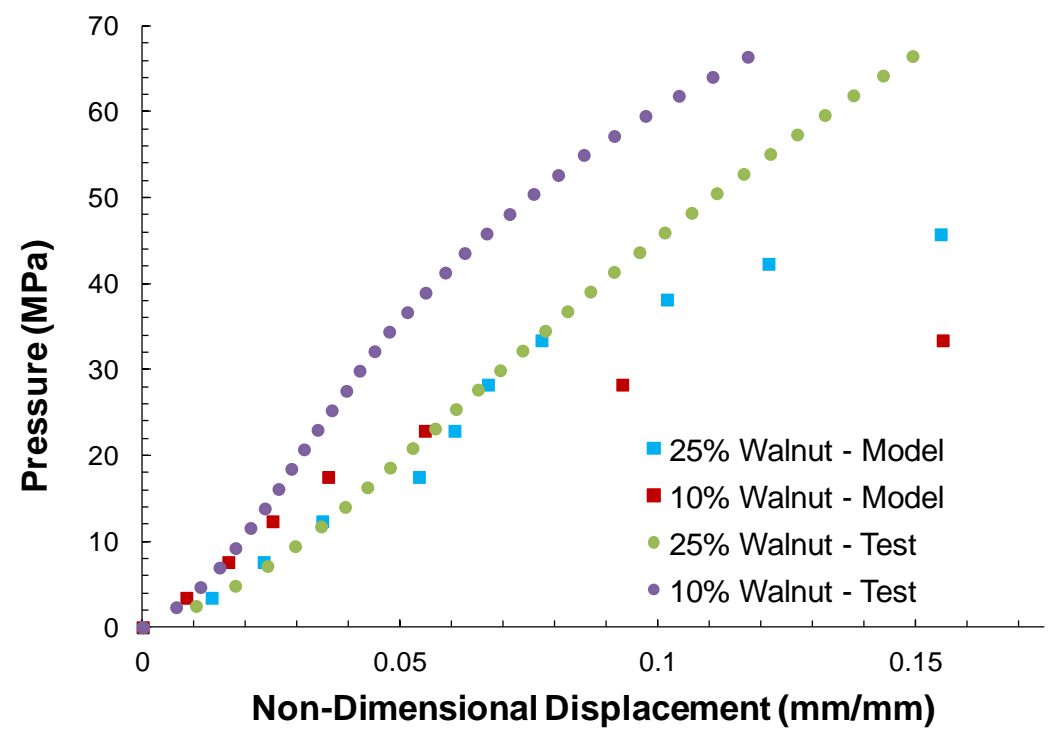

Fig. 5.2. Pressure vs non-dimensional displacement comparison of $10 \%$ and $25 \%$ walnut models with fracture and confine compression/crush tests 


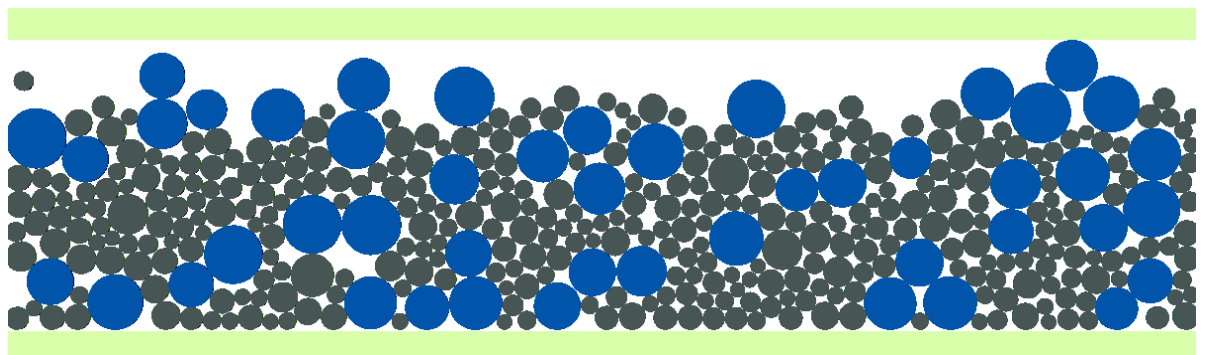

(a)

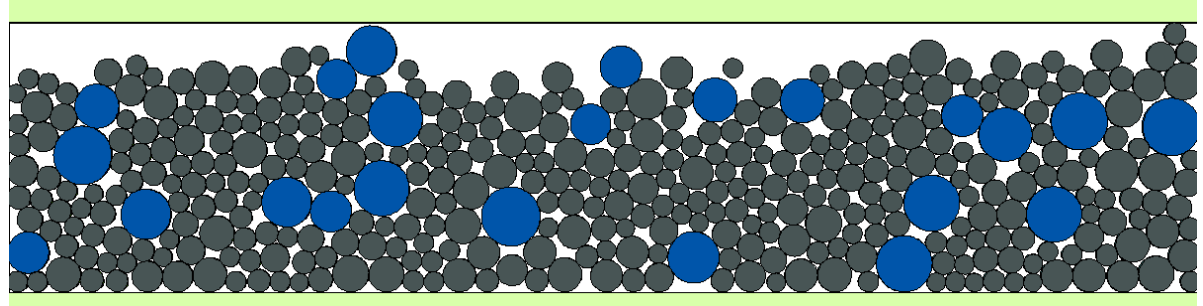

(b)

Fig. 5.3. Initial configuration prior to compressive loading for (a) $25 \%$ walnut - LWC ceramic model and (b) 10\% walnut - LWC ceramic model

Notice that the $10 \%$ model shows deviation at much lower pressure from the test response in comparison to the $25 \%$ model. This is representative of the higher percentage of particle fracture experienced in the $10 \%$ model and is consistent with the particle crush test data. It is noted that consistency here is only qualitative and a quantitative comparison is not possible for the reasons as stated above which result in a weaker response for the models compared to the tests.

At present in the industry, the information on pack porosity and hence permeability is obtained only from very expensive long term conductivity tests. As articulated in section 4.2, the current crush tests also don't have any provision to address pack porosity. One of the advantages computational modeling provides us is the ability to predict the porosity in the model based on its deformed configuration as illustrated in 
the ceramic damage models in section 2 and section 3.2. Herein the variation in porosities of the $25 \%$ and $10 \%$ fracture models with pressure is compared in Fig. 5.4. It can be observed that even though the 10\% model shows higher fragmentation of particles it still possesses higher porosity. Note that the porosity is once again computed from image processing of the deformed configuration. The non-dimensional metric is created as the ratio of the porous area to the original area occupied by the undeformed pack. It can also be observed that around the point where significant particle fracture occurs and the model starts deviating from the test, the $10 \%$ model shows a sudden loss in porosity. Thus it can be argued that even though at lower pressures the $10 \%$ mixture maintains higher stiffness and porosity it could lose its porosity at higher pressures with an increase in particle fracture. The von Mises stress contours for the two models at 33 $\mathrm{MPa}$, the pressure at which significant particle fragmentation is observed in the $10 \%$ walnut models are presented in Fig. 5.5a and 5.5b for $10 \%$ and $25 \%$ walnut models respectively. The higher fraction of fractured ceramic particles is easily observable from the two figures.

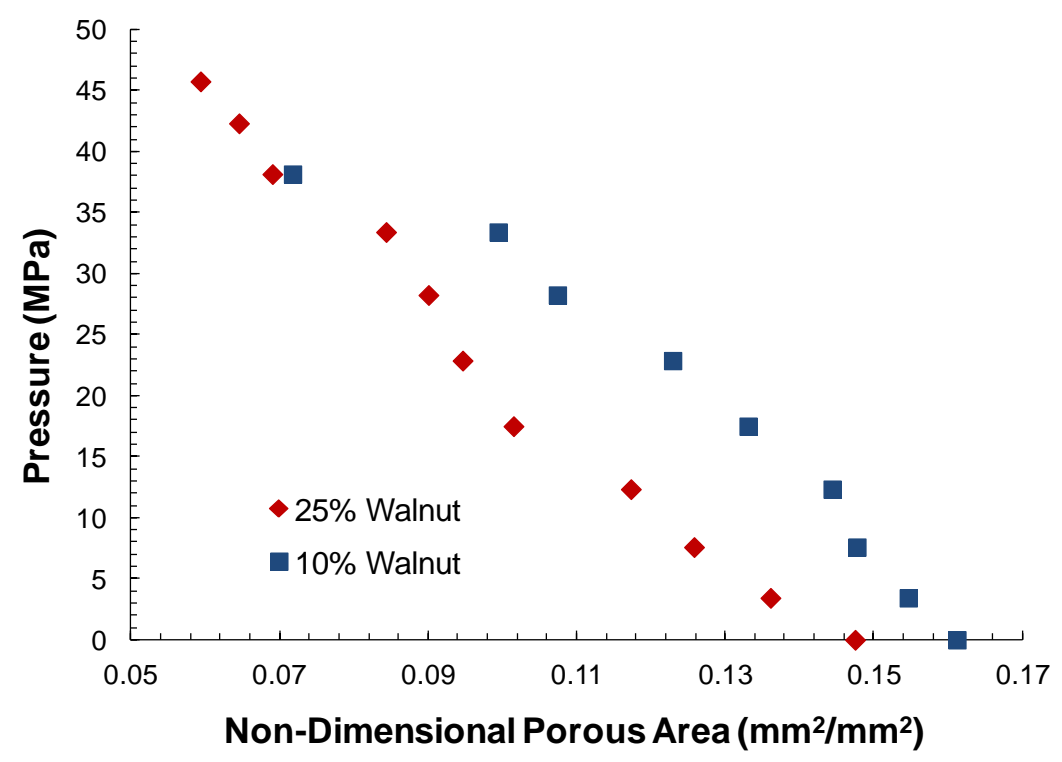

Fig. 5.4. Pressure vs non-dimensional porosity comparison of $10 \%$ and $25 \%$ walnut models with fracture 


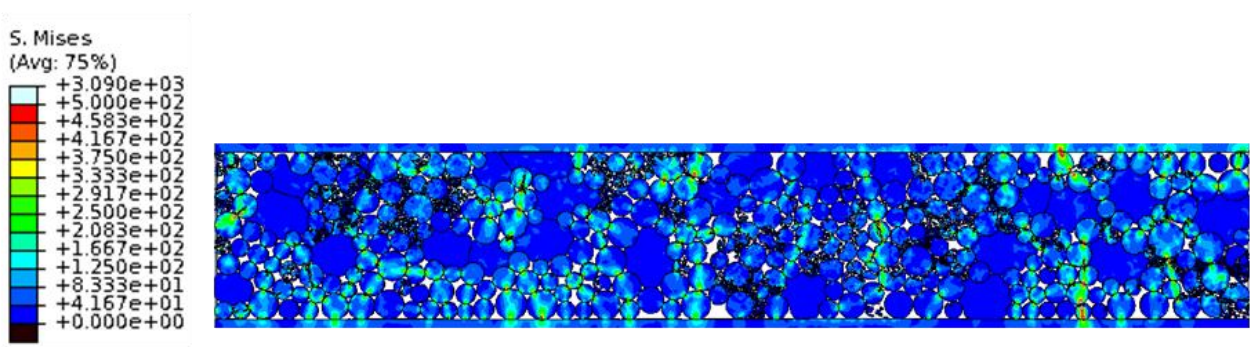

(a)

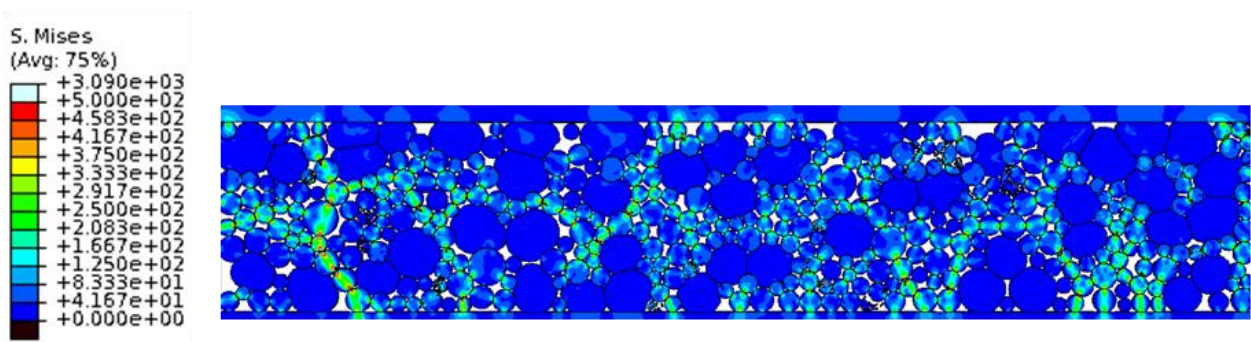

(b)

Fig. 5.5. Von Mises stress contours at $33 \mathrm{MPa}$ pressure for (a) 10\% walnut model with ceramic fracture and (b) $25 \%$ walnut model with ceramic fracture

\subsection{Models with Ceramic Fracture - 25\% Pure Al}

To compare the effect of particle shape and size on pack response in terms of stiffness, particle fracture and porosity, three different combinations of pure Al particles are considered. The weight composition of pure $\mathrm{Al}$ is maintained at $25 \%$. The initial configurations of the three models are presented in Fig. 5.6a, 5.6b and 5.6c. These are classified as large thick needles $((2.5-3.2) \mathrm{X}(0.4-0.8) \mathrm{mm})$, small thin needles $((1.7-$ 2.25) $\mathrm{X}(0.3-0.6) \mathrm{mm})$ and pellets $(0.8-1.2 \mathrm{~mm})$ diameter. The pressure vs nondimensional displacement response of the three models in comparison to each other and the 25\% pure Al needles crush test response are presented in Fig. 5.7. 


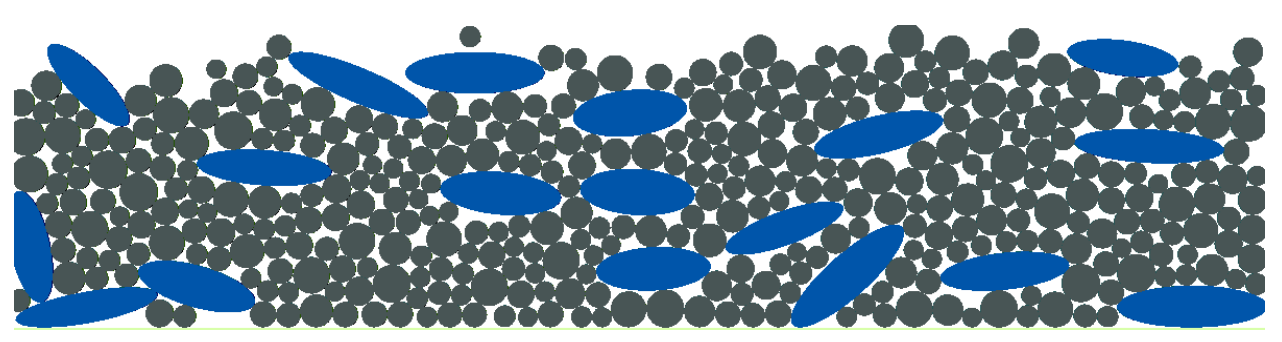

(a)

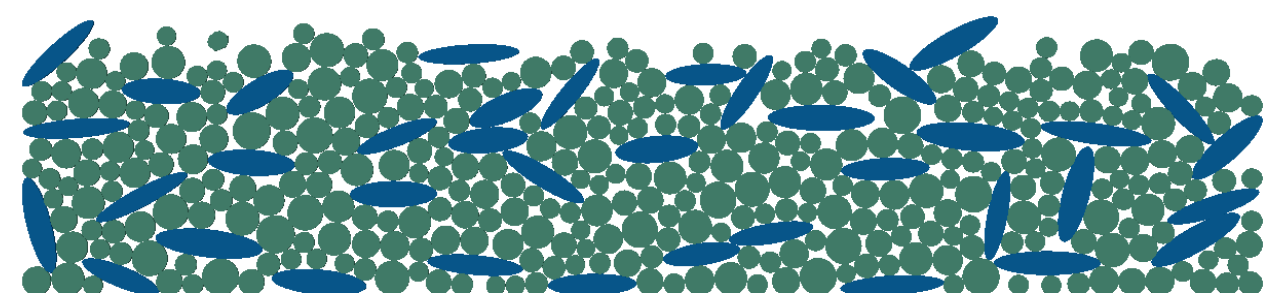

(b)

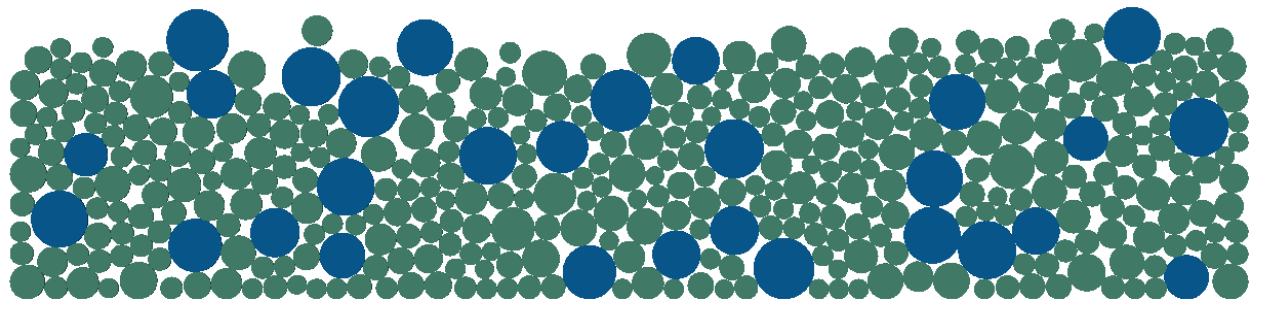

(c)

Fig. 5.6. Initial configuration for the three $25 \%$ pure $\mathrm{Al}-\mathrm{LWC}$ ceramic models (a) large needles (b) small needles and (c) pellets

The small needles provide the stiffest response as shown in Fig 5.7. The large needles offer the softest response which matches closely with the test at lower pressures prior to the onset of significant particle fracture. Recall from section 4.2 the dimension of the pure $\mathrm{Al}$ needles range from (2.5-3.5) X (0.425-0.850) mm which matches closely with the dimension of the large needles. 
The pressure vs non-dimensional porous area response for the three pure Al models is presented in Fig. 5.8. The pure Al models are also compared to the $25 \%$ walnut model. It is observed that the three models show almost similar porosity at all loads, though the small needles model has the highest porosity of all three and the long needles has the least. The pure Al models also show much higher porosity when compared to the $25 \%$ walnut model. Again note the sudden change in porosity at 35-40 $\mathrm{MPa}$ due to the increase in particle fragmentation. In this pressure range, we also observe the softening in the pressure vs non-dimensional displacement response of $\mathrm{Al}$ models.

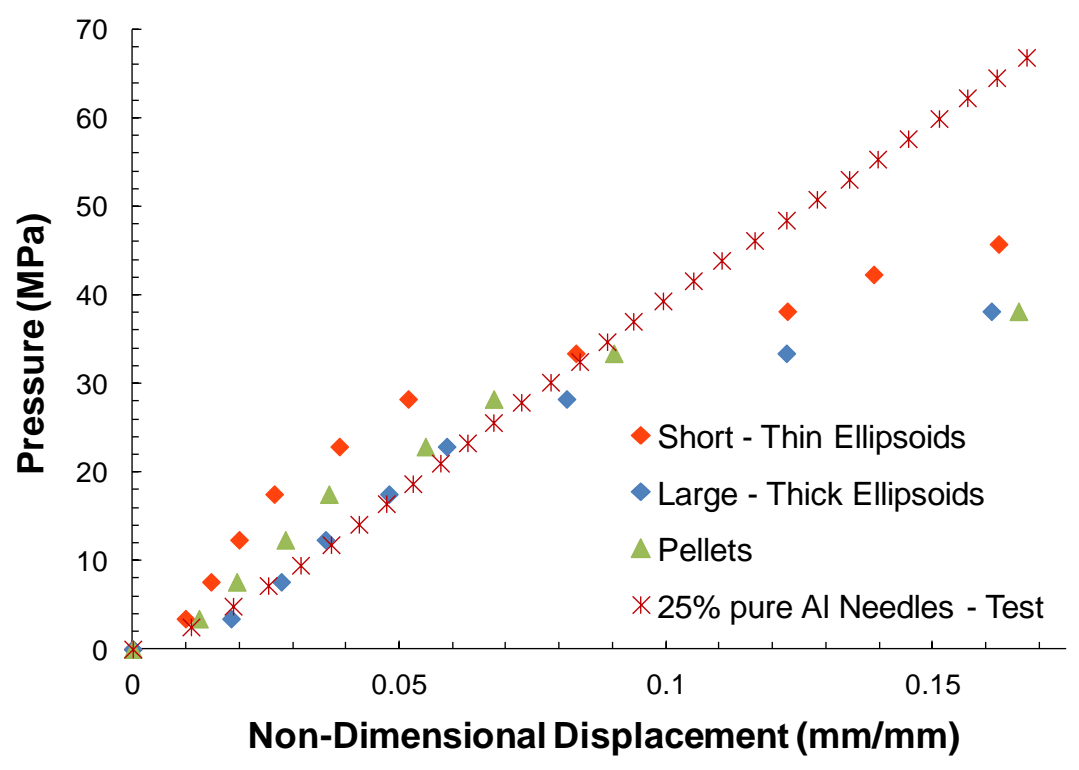

Fig. 5.7. Pressure vs displacement response of $25 \%$ pure Al models, comparison to test results for $25 \%$ pure $\mathrm{Al}$ needles 


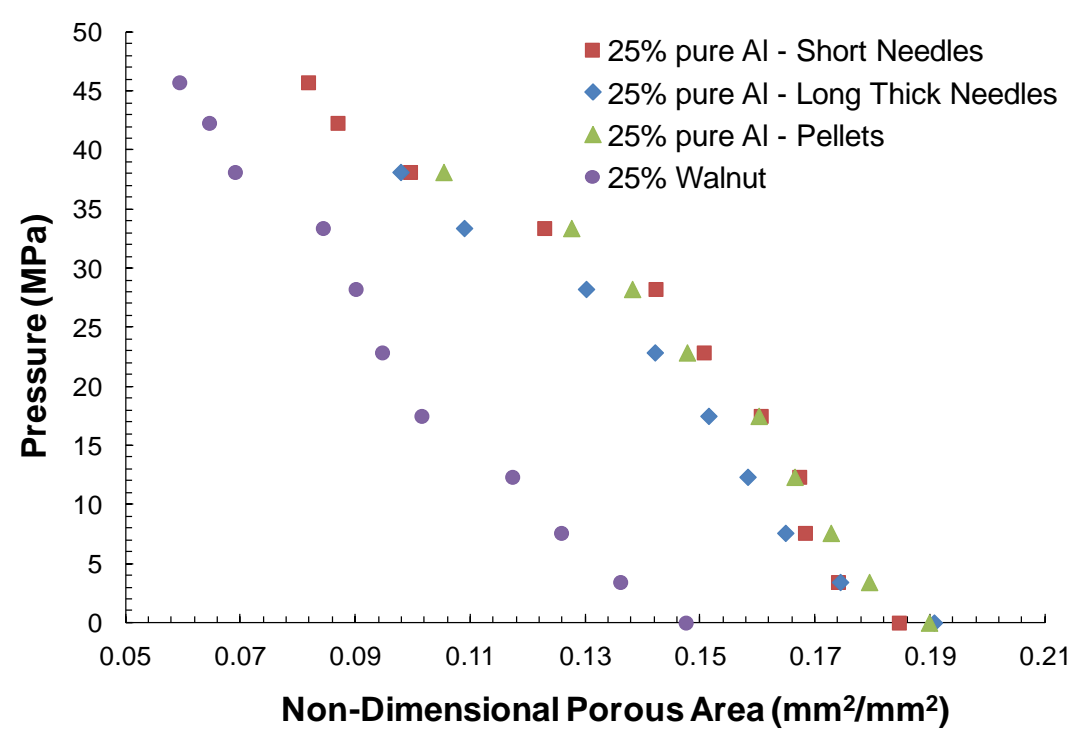

Fig. 5.8. Pressure vs porosity comparison for $25 \%$ pure $\mathrm{Al}$ models and $25 \%$ walnut model

The von Mises stress contours for the three pure Al models at $38 \mathrm{MPa}$ pressure are displayed in Fig. 5.9a, 5.9b and 5.9c. It can be observed that most fragmentation takes place for the large needles model while least is observed for the small needles.
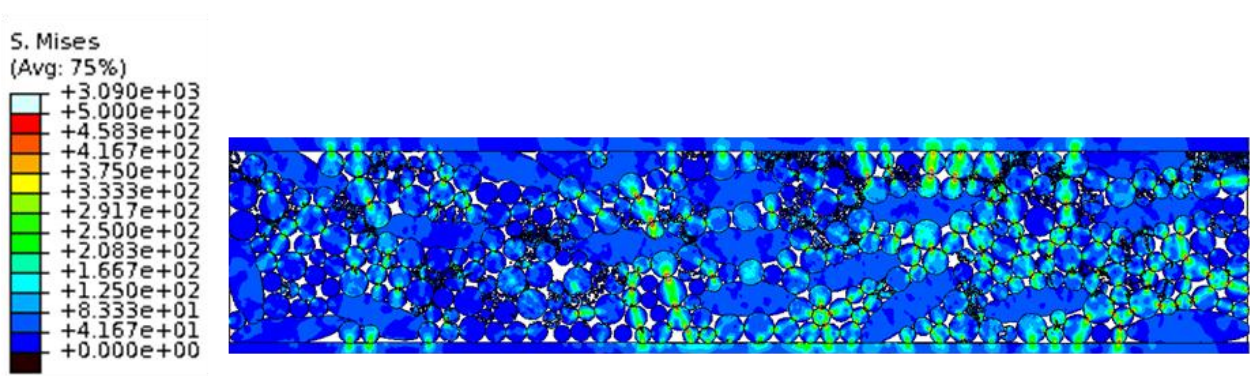

(a)

Fig. 5.9. Von Mises stress contours at $38 \mathrm{MPa}$ pressure for (a) 25\% pure Al large needles model (b) 25\% pure Al small needles model and (c) 25\% pure Al pellets model 

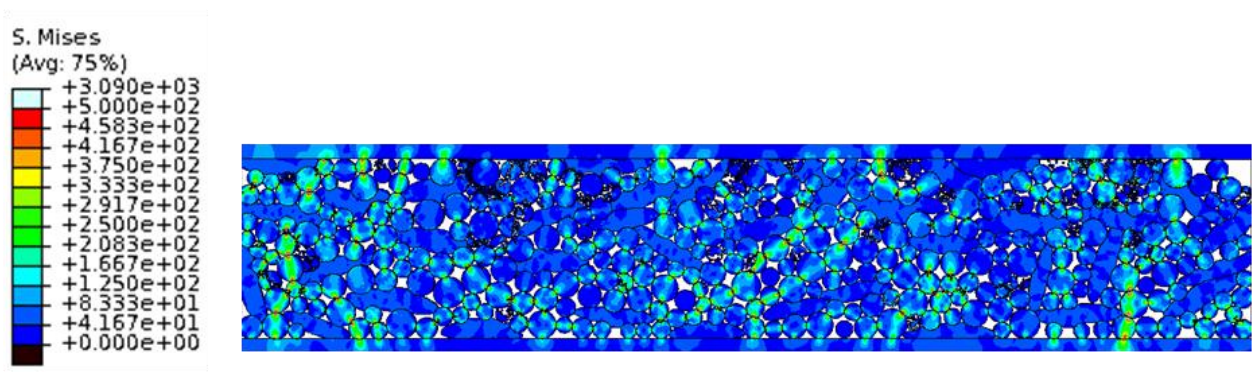

(b)
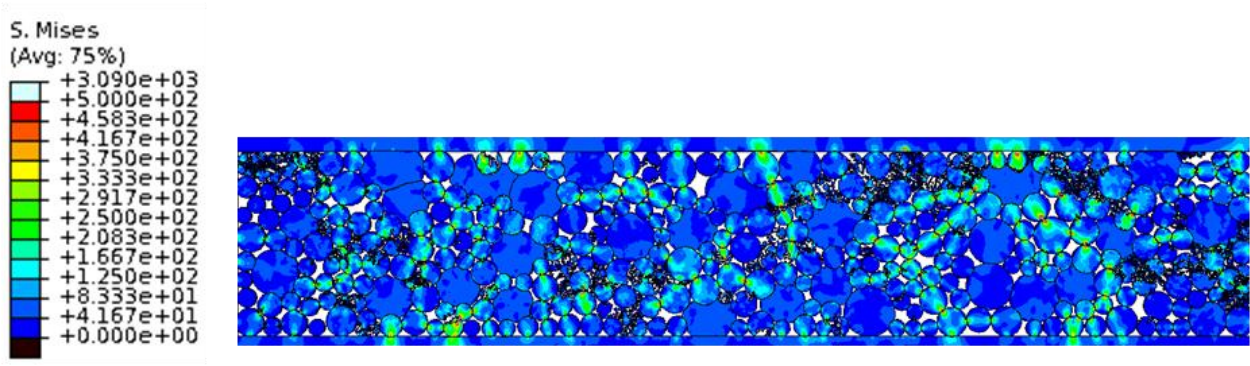

(c)

Fig. 5.9. Continued
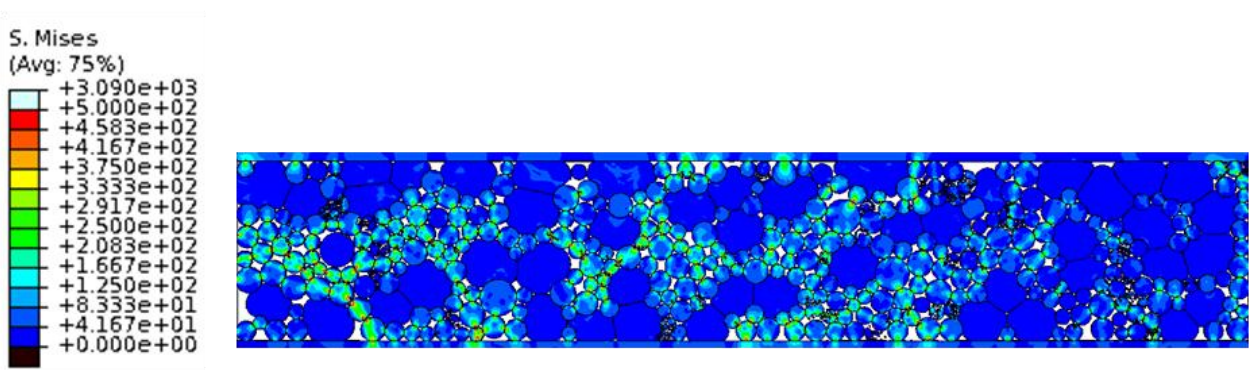

Fig. 5.10. Von Mises stress contour at $38 \mathrm{MPa}$ pressure for $25 \%$ walnut model

As presented in Fig. 5.10 and Fig. 5.9a, the walnut model shows lower particle fragmentation compared to the large needles $25 \%$ pure $\mathrm{Al}$ model at the same pressure of $38 \mathrm{MPa}$. Similarly comparing the pressure vs non-dimensional displacement plots for the two models in Fig. 5.11, we observe that the 25\% pure Al large needles model begins to diverge from the test response at slightly lower pressure than the walnut model. This 
is attributed to the larger number of particles fracturing in the large needles model. Note, even though the $25 \%$ pure Al large needles model shows lower stiffness, it still maintains higher porosity. The porosities reported here may be artificially higher at higher pressures as the fracture is simulated by employing element deletion which results in a loss of volume and consequently in an image will appear as porosity, which will not be the case in reality. Thus at higher pressure we may be getting a higher porosity for the pure $\mathrm{Al}$ models compared to the walnut models primarily due to element deletion. At lower pressures where the element deletion is still not significant, we can be relatively confident with the porosity comparisons.

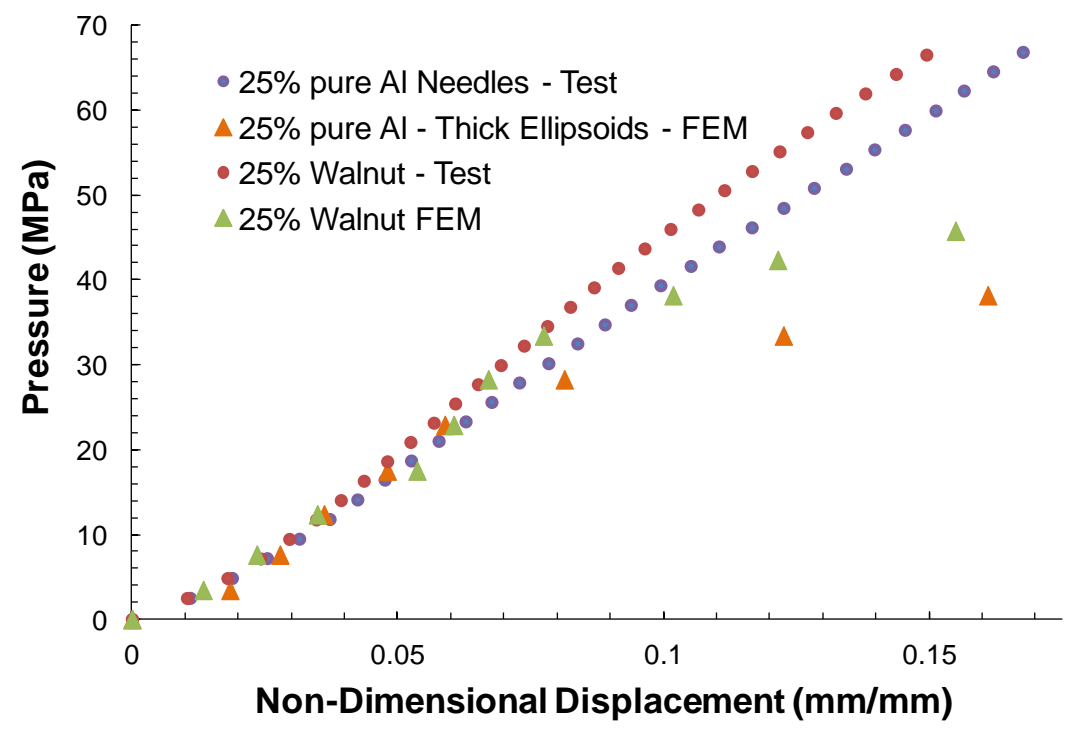

Fig. 5.11. Pressure vs non-dimensional displacement comparison of $25 \%$ pure Al large needle, $25 \%$ walnut model with test data

From the above simulation we can observe that the small needles model give a better response in terms of stiffness, fracture and porosity compared to the large needles model and can be considered for further exploration. The models need to be expanded 
to simulate flowback and then a better comparison of different mixtures can be obtained from point of view of both flowback and compression to enable us to better screen proppant samples.

In conclusion, the models developed offer meaningful and simple comparisons to create different mixtures and screen proppants based on porosity, stiffness and particle fracture. Even though the models do not predict exact values of porosity and fracture they can be further developed as an effective and efficient analytical tool. The models can be further improved by exploring the influence of model dimensions i.e. models with many more particles. Better fracture simulation techniques like the XFEM methods with no element deletion if available in ABAQUS explicit can definitely improve the current models and can better predict pack porosities. Further, better fracture energy values for the ceramic models can lead to improved simulations. In addition the extension to $3 \mathrm{D}$ simulations is recommended in the future studies. 


\section{CONCLUSIONS}

Computational models are developed to tailor the composition and the response of proppants under realistic hydraulic fracture loads; compression loading from rock faces and transverse fluid drag forces. Complementary experiments are designed and conducted to bridge our understanding between laboratory and infield responses essential for successful predictions.

The uniformly sized particle models form the base on which the polydisperse models with particle size distribution are developed. Initially three different mixtures of walnut and ceramic particles are considered within a sixty particle model. The pressure vs displacement response revealed a nonlinear behavior for this granular pack. The pack stiffness is lower for mixtures with higher percent of softer particles. A corresponding reduction in the pack porosity is also observed. Stress chains clearly demonstrate how the load is transferred within a pack through particle interactions. The harder ceramic particles carry most of the load while the softer particles undergo considerable deformation. The flowback models display that the formation of a stable arch is essential to maintain pack stability. The other variables that are found to increase resistance to flowback are as follows: addition of softer particles to a pack, softer rock surfaces and higher inter-particle friction.

The polydisperse models, mixtures of hard (ceramic) and soft (walnut and pure aluminum) particles under confined compression are studied to assess the influence of different materials, shape and size of the particles on the pack response in terms of porosity, pressure vs displacement and particle fracture. A Matlab code employing the random number generation function is developed to assign random particle co-ordinates and particle diameters to generate the initial pack configuration. Two main classes of constitutive behavior for ceramic particles are considered a) damage without fracture and b) damage with fracture. The pressure vs displacement response for all the compositions showed a continuously stiffening nonlinear behavior. Pack porosity is reduced with an increase in the percentage of softer particles. For the same percent composition of soft 
particles, the pure aluminum mixture gives a stiffer response and at the same time displays higher porosity than the walnut particle mixture. Particle rearrangement is limited for packs with higher friction which results in a stiffer pressure vs displacement response and a more porous pack.

For the models in class (b), fragmentation of ceramic particles leads to a softening in the pressure vs displacement curve. The response of these models diverges from the damage models at higher pressures with increase in fragmentation. The response for the $10 \%$ walnut models is stiffer than that of the $25 \%$ model, but the former shows more particle fragmentation. Similar observations are noted for the $25 \%$ pure aluminum model when compared to the $25 \%$ walnut model.

Single particle compression tests on light weight (LWC) ceramic particles are conducted to obtain material properties to be incorporated in the FEM models. Single particle compression test results on pistachio shells are observed to be stiffer compared to walnut. Pistachio particles are also observed to possess a more cuboidal shape compared to the more rounded walnut shell particles. The confined compression/crush tests are conducted for mixtures with LWC, high strength (HSP) and Ottawa sand as the base materials. The pressure vs displacement curves show three distinct phases involving particle rearrangement, consolidation and finally softening due to fracture. It is observed that pistachio-LWC ceramic and pure Al pellets-LWC ceramic mixtures display the least amount of particle crush for all compositions. The pure Al pellets-LWC ceramic mixture is the stiffest. The pure Al needles-LWC ceramic mixture is shows maximum crush of all the mixtures for compositions with more than $10 \%$ soft particles, this is attributed to its longer ellipsoidal shape which results into the disruption of the pack and introduces stress concentration in the ceramic particles. The pistachio mixtures are consistently stiffer than the walnut mixtures at all compositions primarily due to pistachio's stiffer response and more cuboidal shape as a single particle. For all the mixtures with increase in composition of softer particles the particle crush is reduced, except for the $10 \%$ pure Al mixture which shows a slightly higher crush than the $6 \%$ mixture. Similarly, the pack stiffness also shows a decline with increase in percentage 
of softer particles. Similar results are observed for mixtures with both HSP bauxite and Ottawa sand as the base material.

The pressure vs displacement responses for $10 \%$ and $25 \%$ walnut-LWC ceramic and $25 \%$ pure Al-LWC ceramic FEM model all with ceramic fracture are compared with the experimental results from crush tests. The FEM and experimental results are in agreement at lower pressures with divergence observed at higher pressures. This is primarily attributed to a weaker ceramic post cracking material definition which was assumed and not determined experimentally and spurious weakness introduced due to element deletion. Higher crush is observed for the $10 \%$ walnut mixture compared to the $25 \%$ walnut mixture which is consistent with experimental observations. Similarly higher particle fracture is observed for $25 \%$ large needles pure $\mathrm{Al}$ mixtures compared to $25 \%$ walnut mixtures which is also consistent with the experimental observations. The stiffness response of the former is also slightly softer compared to the later which is also consistent with the experimental observations. The increased particle fragmentation for the pure Al mixture lowers the pack stiffness. The pack porosity for $25 \%$ walnut mixture is found to be lower than that of $10 \%$ walnut and the pure $\mathrm{Al}$ mixtures. Thus it is concluded that higher pack stiffness and lower fracture does not necessarily result in higher porosity. The effect of particle shape on the stiffness and porosity of the mixtures is studied by comparing three distinct shapes for pure Al-LWC ceramic mixture. The short-thin needles provide the stiffest response with the long-thick needles provide the softest response. Comparison of pack porosity does not yield a significant difference for each of the three cases.

These results provide a base for further investigations by which better, more efficient particle mixtures can be developed. The short pure Al needles - ceramic and the pistachio - ceramic mixtures are two prime candidates for further investigation. Polydisperse models with particle fracture to simulate flowback need to be developed to further study the influence of different factors like shape, size and material on flowback resistance. Computational modeling provides us with the means to compare pack porosity between different mixture types. The models can be developed further by 
incorporating better fracture properties for ceramics. The models can also be improved by introducing larger models i.e. many more particles, 3D modeling and employing better fracture simulation techniques. 


\section{REFERENCES}

1. Mader D. Hydraulic proppant fracturing and gravel packing. Elsevier Science Publishing Company Inc., New York; 1989.

2. Skinner DR. Introduction to petroleum production, Gulf Pub. Co., Book Division, Houston; 1981.

3. Rickards AR, Brannon HD, Wood WD, Stephenson CJ. High strength ultra lightweight proppant lends new dimensions to hydraulic fracturing applications. SPE Production \& Operations 2006; 21(2):212-221.

4. Brannon HD. Maximizing Fracture Conductivity with Proppant Partial Monolayers: Theoretical Curiosity or Highly Productive Reality, In: Proc. of Society of Petroleum Engineers (U.S.), Technical Conference and Exhibition, Houston, TX, pp. 3569$3591 ; 2004$.

5. Brannon HD, Stephenson CJ, Maharidge R. Personal Communication, BJ Services Inc., Tomball, TX; May 2010.

6. Kaufman PB, Anderson RW, Ziegler M, Neves AR, Parker MA et al. Introducing new ISO/API procedures for proppant testing. In: ATCE Proceedings (U.S.), SPE annual technical conference and exhibition, Anaheim, CA, USA, pp. 3526-3532; 2007.

7. American Petroleum Institute. Recommended practices for testing high-strength proppants used in hydraulic fracturing operations, API RP 60. API Publications, Washington, D.C.; 1995.

8. American Petroleum Institute Exploration and Production Department. Recommended practices for testing sand used in hydraulic fracturing operations, API RP 56, $2^{\text {nd }}$ edition. API Publications, Washington, D.C.;1995.

9. de Gennes PG. Granular Matter: A tentative View. Reviews of Modern Physics 1999; 71(2):374-382.

10. Yu AB. Powder Processing: Models and Simulation. Encyclopedia of Condensed Matter Physics, F. Bassani, G.L. Liedl and P. Wyder (eds), Chapter 556 (Vol. 4, pp.401-414), invited contribution, Elsevier, Oxford; 2005.

11. Duran J. Sands, powders, and grains: an introduction to the physics of granular materials, Springer-Verlag, New York, Inc; 1997. 
12. Jaeger HM, Nagel SR. Physics of the granular state. Science 1992; 255:1523-1531.

13. Nedderman RM. Statics and kinematics of granular materials. Cambridge University Press, New York; 1992.

14. Asgian MI, Cundall PA. Mechanical stability of propped hydraulic fractures: a numerical study. JPT 1995; 47(3):203-208.

15. Milton-Tayler D, Stephenson CJ, Asgian MI. Factors affecting the stability of proppant in propped fractures: results of a laboratory study. In: ATCE Proceedings (U.S.), SPE annual technical conference and exhibition, Washington, DC, USA, SPE24821 pp.569-579; 1992.

16. Johnson KL. Contact Mechanics. Cambridge University Press, Cambridge, UK; 1985.

17. Cundall PA. A Discontinuous Future for Numerical Modeling in Soil and Rock. In: Discrete Element Methods Numerical Modeling of Discontinua, Proceedings at the $3^{\text {rd }}$ International Conference on Discrete Element Methods, Santa Fe, New Mexico, pp. 3-4; 2001.

18. Munjiza A. The combined finite-discrete element method. John Wiley \& Sons Ltd, West Sussex, England; 2004.

19. Zienkiewics OC, Chan AHC, Pastor M, Schrefler BA, Schiomi T. Computational geomechanics with special reference to earthquake engineering. John Wiley and Sons, West Sussex, England; 1999.

20. Powrie W. Soil Mechanics: Concepts and Applications. Taylor \& Francis, New York; 1997.

21. Lambe TW, Whitman RV. Soil Mechanics. John Wiley and Sons, USA; 1969.

22. Raaen AM. Efficient Determination of the Parameters of an Elastoplastic Model. In: Proceedings, SPE/ISRM Rock Mechanics in Petroleum Engineering, Trodheim, Norway, 2:377-283; 1998 .

23. ABAQUS. User's manual, Version, 6.8.3. Hibbitt, Karlsson and Sorensen Inc; 2008.

24. Andrews JS, Khorholt H. Rock mechanical principles help to predict proppant flowback from hydraulic fractures. In: Proceedings, SPE/ISRM Rock Mechanics in Petroleum Engineering, Trodheim, Norway, 2:381-390; 1998. 
25. Oda M, Iwashata K. Mechanics of granular materials: an introduction. A. A. Balkema Publishers, Brookfield, VT, USA; 1999.

26. Lin CL, Miller JD. Pore structure and network analysis of filter cake. Chemical Engineering Journal 2000; 80:221-231.

27. Rosato A, Prinz F, Standburg KJ, Swendsen R. Monte carlo simulation of particulate matter segregation. Powder Technology 1986; 49:59-69.

28. Komodromos P, Williams JR. Development and implementation of a combined discrete and finite element multibody dynamics simulation environment. PhD Thesis, Massachusetts Institute of Technology; 2001.

29. Salot C, Gotteland P, Villard P. Influence of relative density on granular materials behavior: dem simulations of triaxial tests. Granular Matter 2009; 11:221-236.

30. Ouwerkerk CED. A micro-mechanical connection between the single particle strength and the bulk strength of random packings of spherical particles. Powder Technology 1991; 65:125-38.

31. O'Sullivan C, Liang C. Micromechanics of granular material response during load reversals: combined DEM and experimental study. Powder Technology 2009; 193:289-302.

32. Zhao X, Evans TM. Discrete simulations of laboratory loading conditions. International Journal of Geomechanics 2009; 9(4):169-78.

33. Li J, Webb C, Pandiella SS, Campbell GM. Discrete particle motion on sieves - a numerical study using the DEM simulation. Powder Technology 2003; 133:190202.

34. Datta A, Mishra BK., Das SP, Sahu A. A DEM analysis of flow characteristics of noncohesive particles in hopper. Materials and Manufacturing Processes 2008; 23:196-203.

35. Cleary PW, Sawley ML. DEM modelling of industrial granular flows: $3 \mathrm{~d}$ case studies and the effect of particle shape on hopper discharge. Applied Mathematical Modeling 2002; 26:89-111.

36. Lu LS, Hsiau SS. DEM simulation of particle mixing in a sheared granular flow. Particulogy 2008; 6(6):445-454. 
37. Lewis RW, Gethin DT, Yang XS, Rowe RC. A combined finite-discrete element method for simulating pharmaceutical powder tableting. Int J Numer Method Eng 2005; 62:853-69.

38. Gethin DT, Ransing RS, Lewis RW, Dutko M, Crook AJL. Numerical comparison of a deformable discrete element model and an equivalent continuum analysis for the compaction of ductile porous material. Comput. Struct. 2001; 79:1287-1294.

39. Freening G. (2007). Analysis of pharmaceutical powder compaction using multiplicative hyperelasto-plastic theory. Powder Technol. 2007; 172:103-112.

40. Zavaliangos A. A multiparticle simulation of powder compaction using finite element discretization of individual particles. In: Mat. Res. Soc. Symp. Proc., Materials Research society, 731:W7.1.1-W7.1.7; 2002.

41. Zavaliangos A. A numerical study of the development of tensile principle stress during die compaction. Particulate Science and Technology 2003; 21:105-15.

42. Procopio AT, Zavaliangos A. Simulation of multi-axial compaction of granular media from loose to high relative densities. Journal of the Mechanics and Physics of Solids 2005; 53(7):1523-51.

43. Zhang J. A study of compaction of composite particles by multi-particle finite element method. Composites Science and Technology 2009; 69:2048-53.

44. Choi JL, Gethin DT. A discrete finite element modeling and measurements for powder compaction. Modelling Simul. Mater. Sci. Eng. 2009; 17:035005(22pp).

45. Kabir MA, Jasti VK, Higgs CF, Lovell MR. An evaluation of the explicit finiteelement method approach for modelling dense flows of discrete grains in a couette shear cell. In: Proc. IMechE, J.Engineering Tribology - Special Issue paper, $222: 715-723 ; 2008$.

46. Kabir MA, Lovell MR, Higgs CF III. Utilizing the explicit finite element method for studying granular flows. Tribol. Lett. 2008; 29(2):85-94.

47. Frenning G. An efficient finite/discrete element procedure for simulating compression of 3d particle assemblies. Comput. Methods Appl. Mech. Eng. 2008; 197:4266-4272.

48. Frenning G. Compression mechanics of granule beds: a combined/discrete element study. Chemical Engineering Science 2010; 65:2464-71. 
49. Card RJ, Howard PR, Feraud JP. A novel technology to control proppant backproduction. SPE Production \& Facilities 1995; SPE 31007, 271-276.

50. Stephenson CJ, Ward B, Taylor DM, Brannon HD, Rickards AR. Exceptional proppant flowback control for the most extreme well environments: the shape of things to come. In: SPE annual technical conference and exhibition, San Antonio, TX, 29 September - 2 October, SPE77681; 2002.

51. Parker M, Weaver J, Van Bantenburg D. Understanding proppant flowback. In: ATCE Proceedings (U.S.), SPE annual technical conference and exhibition, Houston, TX, USA, SPE56726 pp.1-13; 1999.

52. Vreeburg RJ, Roodhart LP, Davies DR, Penny GS. Proppant backproduction during hydraulic fracturing - a new failure mechanism for resin-coated proppants. JPT 1994; 46(10):884-889.

53. Stephenson CJ, Rickards AR, Brannon HD. Increased resistance to proppant flowback by adding deformable particles to proppant pack tested in the laboratory. In: SPE annual technical conference and exhibition, Houston, TX, USA, SPE56593 pp.1-12; 1999.

54. Rickards AR, Lacy L, Brannon HD, Stephenson CJ, Bilden D. Need stress relief? A new approach to reducing stress cycling induced proppant pack failure. In: SPE annual technical conference and exhibition, New Orleans, Louisiana, USA, SPE49247 pp.1-15; 1998.

55. Reinicke A, Rybacki E, Stanchits S, Huenges E, Dresen G. Hydraulic fracturing stimulation techniques and formation damage mechanisms-implications from laboratory testing of tight sandstone-proppant systems. Chemie der Erde 2010; 70(S3):107-117.

56. Freeman ER, Anschutz DA, Rickards AR, Callanan MJ. Modified API/ISO crush tests with a liquid-saturated proppant under pressure incorporating temperature, time, and cyclic loading: what does it tell us? In: SPE hydraulic fracturing technology conference, Woodlands, TX, USA, SPE118929 pp.1-7; 2009.

57. Blakeley DM. Flow characteristics of hydraulic fracture proppants subjected to repeated production cycles. 1988, M.S. Thesis, Texas A\&M University: College Station, TX.

58. Weaver J, Rickman R, Luo H, Loghry R. A study of proppant-formation reactions. In: SPE international symposium on oilfield chemistry, Woodlands, Texas, USA, SPE121465 pp.1-21; 2009. 
59. Kulkarni MC. Characterization of light weight composite proppants 2008, M.S. Thesis, Texas A\&M University, College Station, TX.

60. Kulkarni MC, Ochoa OO. Light weight composite proppants: computational and experimental study. Mechanics of Advanced Materials and Structures, Accepted for publication 2010 June, DOI: 10.1080/15376494.2011.572241.

61. Lee J, Fenves G. Plastic-damage model for cyclic loading of concrete structures. Journal of Engineering Mechanics 1998; 124(8):892-900.

62. Image J. Image processing and analysis in Java. http://rsb.info.nih.gov/ij/ - Accessed July 2009.

63. Sarzynski MD. Developing and tailoring multi-functional carbon foams for multifield response 2008, PhD Thesis, Texas A\&M University, College Station, TX.

64. Forgerini FL, Figueiredo W. Random deposition of particles of different sizes. Physical Review E 2009; 79(041602):1-6.

65. Meakin P, Ramanlal P, Sander LM, Ball RC. Ballistic deposition on surfaces. Physical Review A 1986; 34(6):5091-5103.

66. Sadhukhan S, Dutta T, Tarafdar S. Pore structure and conductivity modeled by bidisperse ballistic deposition with relaxation. Modelling Simul. Mater. Sci. Eng. 2007; 15:773-786.

67. Munjiza A, Latham JP. Comparison of experimental and FEM/DEM results for gravitational deposition of identical cubes. Engineering Computations 2004; 2(2/3/4):249-264.

68. Latham JP, Munjiza A. The modeling of particle systems with real shapes. Phil. Trans. R. Soc. Lond. A 2004; 362:1953-1972.

69. Askeland D. The science and engineering of materials. $3^{\text {rd }}$ edition, PWS Publishing Company, MA; 1994.

70. Shah SP, Swartz SE, Ouyang C. Fracture mechanics of concrete, Applications of fracture mechanics to concrete, rock and other quasi-brittle materials. John Wiley \& Sons, Inc., NY; 1995.

71. Lee HC, Choi JS, Jung KH, Im YT. Application of element deletion method for numerical analysis of cracking. Journal of Achievements in Materials and Manufacturing Engineering 2009; 35(2):154-161. 
72. Subbiah S, Melkote SN. Evidence of ductile tearing ahead of the cutting tool and modeling the energy consumed in material separation in micro-cutting. Journal of Engineering Materials and Technology 2007; 129:321-331.

73. Lee DW, Ma Z-D, Kikuchi N. FOA (first-order analysis) model of a granule-filled tube for vehicle crash energy absorption. Mechanics of materials 2009; 41: 684-690.

74. Song JH, Wang H, Belytschko T. A comparative study on finite element methods for dynamic fracture. Comput Mech. 2008; 42:239-250.

75. Hillerborg A, Modeer M, Petersson PE. Analysis of crack formation and crack growth in concrete by means of fracture mechanics and finite elements. Cement and Concrete Research 1976; 6:773-782.

76. Mencik J. Strength and fracture of glass and ceramics. Elsevier Science Publishing Company, New York; 1992.

77. Munz D, Fett T. Ceramics: Mechanical properties, failure behavior, materials selection. Springer-Verlag Berlin, Berlin Heidelberg; 1999.

78. Carbo Ceramics. http://www.carboceramics.com/ceramic-proppant/ - Accessed May 2011.

79. Breval E, Jennings JS, Komarneni S, Macmillan NH, Lunghofer EP. Microstructure, strength and environmental degradation of proppants. Journal of Materials Science $1987 ; 22: 2124-2134$.

80. Heinstein MW, Mello FJ, Attaway SW, Larsen TA. Contact-impact modeling in explicit transient dynamics. Comput. Methods Appl. Mech. Engrg. 2000; 187:621640.

81. Lubliner J. Plasticity theory. Dover Publications, Inc., New York, 2008. 


\section{APPENDIX A}

For the proppant problem which is studied here the modeling of interaction between a large number of discrete particles plays an important role. Contact modeling involves two basic aspects a) contact detection and b) contact interaction. Contact detection includes determining possible contact surfaces in a computationally efficient manner and tracking their relative motion. Contact interaction follows contact detection and it includes evaluation of contact forces between two surfaces $[18,80]$. Here a brief summary of contact in ABAQUS explicit and its implementation in the input file is provided.

In case of ABAQUS explicit two algorithms are available to model contact interaction. In case of contact pair algorithm, possible surface pairs which can contact each other need to be identified as part of model or history definition. A second algorithm is the general contact algorithm which allows for a much simplified definition of contact surfaces. In case of general contact a single surface can span multiple unconnected bodies. The contact is then defined as a self contact for this single surface, self contact of a single surface over multiple unconnected bodies automatically incorporates contact between discrete bodies and self contact for a single body. ABAQUS explicit allows for two different algorithms for contact detection and tracking, a small-sliding algorithm which is available only with the contact pair algorithm and a second finite-sliding algorithm which is available in both the algorithms.

Small-sliding algorithm assumes that there is relatively little sliding of the contacting surface against each other. In case the problem is geometrically nonlinear then this tracking approach allows for large rotation, but it is still assumed that a designated slave surface node will always interact with the same area of the master surface during the entire duration of the analysis. With a finite-sliding algorithm the two contact surfaces are allowed to undergo arbitrary relative separation, sliding and rotation. The contact constraints change with tangential motion of the contacting surfaces.

In case of general contact in ABAQUS explicit, finite-sliding is the default algorithm. The contact discretization of the contact pair surfaces follows a surface-to- 
surface discretization approach. With this approach contact conditions are enforced in an average sense or regions near a slave node which are approximately centered on the slave nodes rather than only at an individual slave node. The contact constraints still consider one slave node predominantly but they also consider the adjacent slave nodes. With this approach some penetration may be observed but large penetrations of the master surface into the slave surface which might go undetected do not occur. The direction of the contact normal is an average of the slave surface in the region around a slave node. From the point of view of contact pressures and stresses surface-to-surface discretization provides more accurate results compared to the node-to-surface discretization. A smoothing effect is observed with the former approach as penetration is resisted in an average sense over a finite region of the slave surface.

ABAQUS explicit employs two algorithms to enforce contact constraints and to calculate contact forces. The Kinematic contact algorithm is only available with the contact pair approach. The general contact approach enforces contact constraints using the penalty contact algorithm. With this approach a slight penetration of the master surface into the slave surface is allowed. The algorithm first searches for penetrations of nodes into the faces in a given configuration and then an opposing force which is dependent on the penetration distance is applied to the slave and master surfaces to oppose the penetration. The force is applied as a distributed load on the nodes forming the contact faces. In case of general contact a balanced master-slave approach is employed for contact constraint enforcement. With this approach the two contacting surfaces are alternatively considered as master and slave and contact forces are computed twice accordingly. Finally a weighted average of the two values is applied as a resultant contact force on both the surfaces. The weighting depends on the defined weighting factor specified for the surfaces; the default is to weight the two sets equally. The penalty parameter can be considered to be as equivalent to that of elastic spring stiffness and can be both linear and nonlinear. This stiffness for general contact is chosen automatically by ABAQUS explicit and is dependent on the stiffness of the underlying element. As values of penalty stiffness increase the penetration is reduced 
but it can lead to a decrease in the stable time increment. One of the advantages of penalty approach over the kinematic contact is that energy is not dissipated for the former. With the kinematic approach for coarse meshes the energy dissipation is significant. The advantage of hard kinematic approach over the penalty approach is that no penetration of the slave surface is allowed into the master surface, though the master can have some penetration for a coarse slave surface. General contact considers element based surfaces hence it becomes relatively easy to define contact on newly generated surfaces because of element deletion. It also has built in smoothing to for element based surfaces for better contact enforcement at corners. One limitation of general contact is its limitation to 3D surfaces for ABAQUS explicit. From an implementation perspective, the keywords necessary to implement general contact as part of model definition and their sequence are presented below. The first set describes the application for noneroding surfaces i.e. no particle fracture.

*SURFACE INTERACTION, NAME=FRIC

*FRICTION

0.3 ,

*CONTACT

*CONTACT INCLUSIONS, ALL EXTERIOR

*CONTACT PROPERTY ASSIGNMENT

, , FRIC

Note, here the first two lines represent contact property definitions mainly the coefficient of friction (here 0.3 ) which is implemented as a form of Coulomb friction in ABAQUS explicit. The keyword * Contact defines general contact. All Exterior, as the definition includes all the external faces of elements in the domain for contact interaction and enforcement. Finally the contact property defined earlier is assigned to the defined contact domain. 
For the general contact if an appropriate interior surface is defined then the contact region can evolve based on the failure of external surface elements which exposes the underlying element faces. General contact considers a face for defining contact only if its underlying element has not failed. Once an element fails, its faces and edges are removed from the analysis the contact domain is modified by defining the contact surface on the face of the newly exposed element. The keywords listed below first define an interior surface and then define an evolving general contact based on element deletion. Here the interior surface is defined in the interior of element set Ceramic and assigned a name surfl.

*SURFACE, TYPE = ELEMENT, NAME $=$ surf1

Ceramic, interior

*SURFACE INTERACTION, NAME = FRIC

*FRICTION

0.3 ,

*CONTACT

*CONTACT INCLUSIONS

,

, surf1

surf1,

*CONTACT CONTROLS ASSIGNMENT, NODAL EROSION = YES

*CONTACT PROPERTY ASSIGNMENT

, FRIC 


\section{APPENDIX B}

In the research presented in here ceramic particles are modeled using a) concrete damage plasticity model and b) concrete brittle cracking model. Here a brief overview of both these models as described in the Abaqus explicit v 6.8.3 user's manual is provided. Ceramic is generally considered to be a brittle material but in certain cases has been reported to show a quasi-brittle response with a rising R-curve [70, 77]. Also the Abaqus user's manual states that though the two models have been developed primarily to simulate reinforced and unreinforced concrete they can be used to model quasi-brittle material like certain ceramics and rocks. The response of concrete, rock or ceramic type quasi-brittle materials differs from those of the classically brittle materials like cast iron or glass which fracture almost immediately as the proportionality limit is reached. For the quasi-brittle materials the stress reduces gradually once the peak is reached. The failure strains are still small generally less than 1\% [81]. The onset of fracture occurs when the stress strain curve reaches its peak while the gradual reduction of the slope represents a reduction in stiffness due to progressive cracking.

\section{Concrete Damage Plasticity}

In this model the inelastic behavior of ceramic is modeled using the concepts of isotropic damaged elasticity combined with isotropic tensile and compressive plasticity. The two main failure modes considered here are tensile cracking and compressive crushing of the concrete material. The responses for this model in tension and compression are presented in Fig. B1 and B2. Under uniaxial tension the stress initially rises linearly until the failure stress is reached. From this point onwards the stress reduces continuously due to the formation of micro cracks and is represented as a softening stress-strain response. This results in strain localization in the structure. Under uniaxial compression the stress-strain response is initially linear until it reaches initial yield stress. From here on we obtain a stress-hardening response in the stress- 
strain curve which is again due to the formation of diffused micro cracks in the structure parallel to the loading direction. Beyond the ultimate stress the compressive response displays a softening stress-strain curve. Note that the reason for stress-strain softening is micro cracking which is represented as permanent plastic deformation.

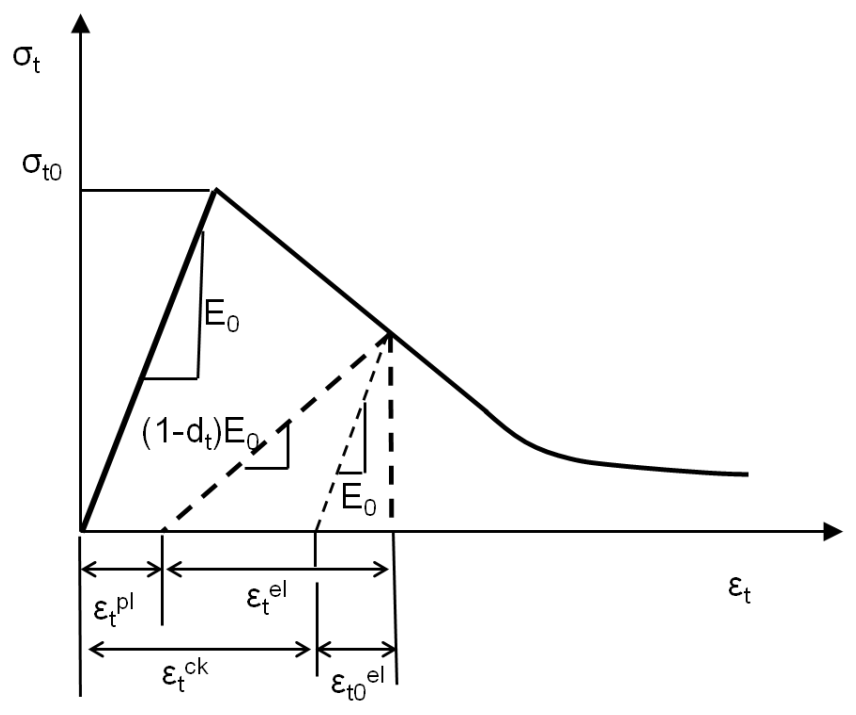

Fig. B1. Response of concrete damage plasticity model under uniaxial tension

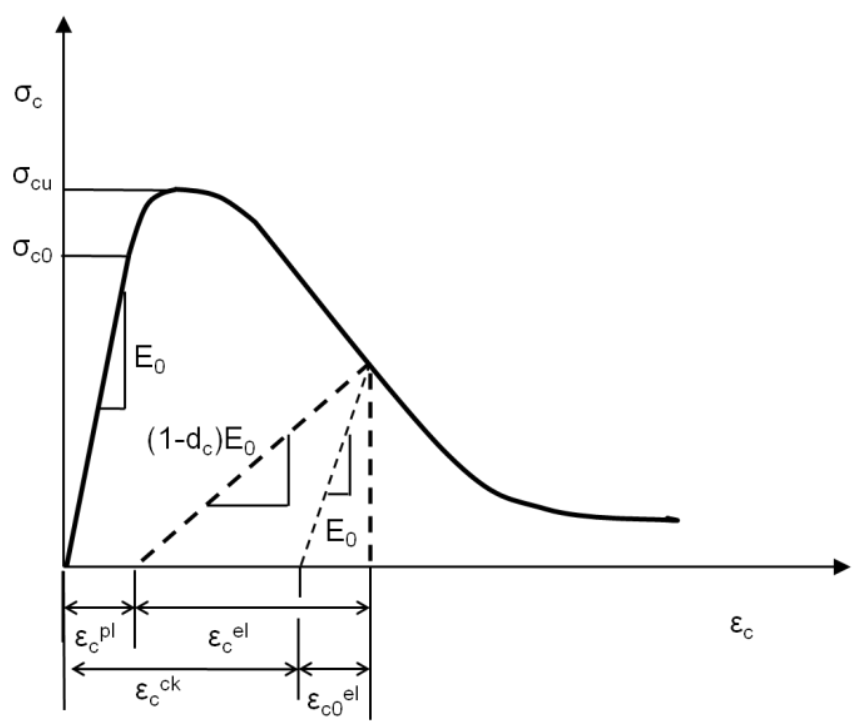

Fig. B2. Response of concrete damage plasticity model under uniaxial compression 
ABAQUS converts the stress-strain curves above into stress vs plastic strain curves. It can be observed that whenever a specimen is unloaded from a point on the softening portion of the stress-strain curve it loses its stiffness. This degradation of the elastic stiffness is represented by two damage variables $d_{c}$ and $d_{t}$ for compression and tension respectively. These are functions of plastic strains, temperature and field variables. The values of the damage variables range from 0 (undamaged) to 1 (complete loss of strength). If $\mathrm{E}_{0}$ is the initial undamaged elastic modulus the stress-strain relations under uniaxial tension and compression are given by

$$
\begin{aligned}
& \sigma_{\mathrm{t}}=\left(1-\mathrm{d}_{\mathrm{t}}\right) * \mathrm{E}_{0} *\left(\varepsilon_{\mathrm{t}}-\varepsilon_{\mathrm{t}}{ }^{\mathrm{pl}}\right) \\
& \sigma_{\mathrm{c}}=\left(1-\mathrm{d}_{\mathrm{c}}\right) * \mathrm{E}_{0} *\left(\varepsilon_{\mathrm{c}}-\varepsilon_{\mathrm{c}}{ }^{\mathrm{pl}}\right)
\end{aligned}
$$

The effective compressive and tensile cohesive stresses are defined as follows. These two stresses determine the size of the yield or failure surface.

$$
\begin{aligned}
& \sigma_{\mathrm{te}}=\sigma_{\mathrm{t}} /\left(1-\mathrm{d}_{\mathrm{t}}\right)=\mathrm{E}_{0} *\left(\varepsilon_{\mathrm{t}}-\varepsilon_{\mathrm{t}}^{\mathrm{pl}}\right) \\
& \sigma_{\mathrm{ce}}=\sigma_{\mathrm{c}} /\left(1-\mathrm{d}_{\mathrm{c}}\right)=\mathrm{E}_{0} *\left(\varepsilon_{\mathrm{c}}-\varepsilon_{\mathrm{c}}^{\mathrm{pl}}\right)
\end{aligned}
$$

The post failure behavior for concrete model is defined in ABAQUS in terms of post failure stress vs cracking strain data. Cracking strain $\left(\varepsilon_{\mathrm{t}}^{\mathrm{ck}}\right)$ in tension is defined in Fig. B1. Cracking strain is equal to the total strain minus the undamaged elastic strain of the specimen $\left(\varepsilon_{\mathrm{t}}{ }^{\mathrm{ck}}=\varepsilon_{\mathrm{t}}-\varepsilon_{\mathrm{t} 0}{ }^{\mathrm{el}}\right)$. The undamaged elastic strain is defined as $\varepsilon_{\mathrm{t} 0}{ }^{\mathrm{el}}=\sigma_{\mathrm{t}} / \mathrm{E}_{0}$. The unloading data if available are provided to ABAQUS in terms of tensile damage cracking strain curves, $\left(d_{t}-\varepsilon_{t}^{c k}\right)$. ABAQUS then converts the cracking strain value to plastic strain using the following relationship. The plastic strain can then be employed to determine the cohesive stresses and yield surface.

$$
\varepsilon_{\mathrm{t}}^{\mathrm{pl}}=\varepsilon_{\mathrm{t}}^{\mathrm{ck}}-\left[\mathrm{d}_{\mathrm{t}} /\left(1-\mathrm{d}_{\mathrm{t}}\right) *\left(\sigma_{\mathrm{t}} / \mathrm{E}_{0}\right)\right]
$$


It has been observed that with unreinforced structures a stress-strain post failure data gives spurious mesh sensitivity. The recommended practice is to define the post failure data in terms of fracture energy $\left(\mathrm{G}_{\mathrm{f}}\right)$. There are two approaches to this one is to define fracture energy directly while the other is to define the data in terms of stresscrack opening. This approach eliminates mesh sensitivity as the crack opening is independent of specimen size.

As with the tension data the compressive post failure data is provided in terms of stress vs inelastic compressive strains $\left(\varepsilon_{\mathrm{c}}{ }^{\mathrm{ck}}\right)$. Note that positive values are used to define the stress strain response in compression. The inelastic compressive strain is defined as a subtraction of total compressive strain and the undamaged compressive strain, $\left(\varepsilon_{\mathrm{c}}{ }^{\mathrm{ck}}=\varepsilon_{\mathrm{c}}\right.$ $\left.-\varepsilon_{\mathrm{c} 0}{ }^{\mathrm{el}}\right)$. And the compressive undamaged elastic strain is defined as, $\varepsilon_{\mathrm{c} 0}{ }^{\mathrm{el}}=\sigma_{\mathrm{c}} / \mathrm{E}_{0}$. As with tension the inelastic compressive strain is converted to the plastic compressive strain.

$\mathrm{E}_{\mathrm{c}}^{\mathrm{pl}}=\varepsilon_{\mathrm{c}}^{\mathrm{ck}}-\left[\mathrm{d}_{\mathrm{c}} /\left(1-\mathrm{d}_{\mathrm{c}}\right) *\left(\sigma_{\mathrm{c}} / \mathrm{E}_{0}\right)\right]$

The concrete damage plasticity model assumes nonassociated plastic flow with the flow potential being the Drucker-Prager hyperbolic function. Furthermore the yield function employed is the one takes into account the different evolution of strength under tension and compression.

The implementation of this material model in ABAQUS input file for the models described in section 3.2 with ceramic damage is presented below. Note that here we have not defined the softening behavior in compression, and the tension softening is defined in terms of stress-displacement relationship.

*MATERIAL, NAME=ceramic

*DENSITY

3.6000E-06,0.0

*ELASTIC, TYPE = ISOTROPIC 


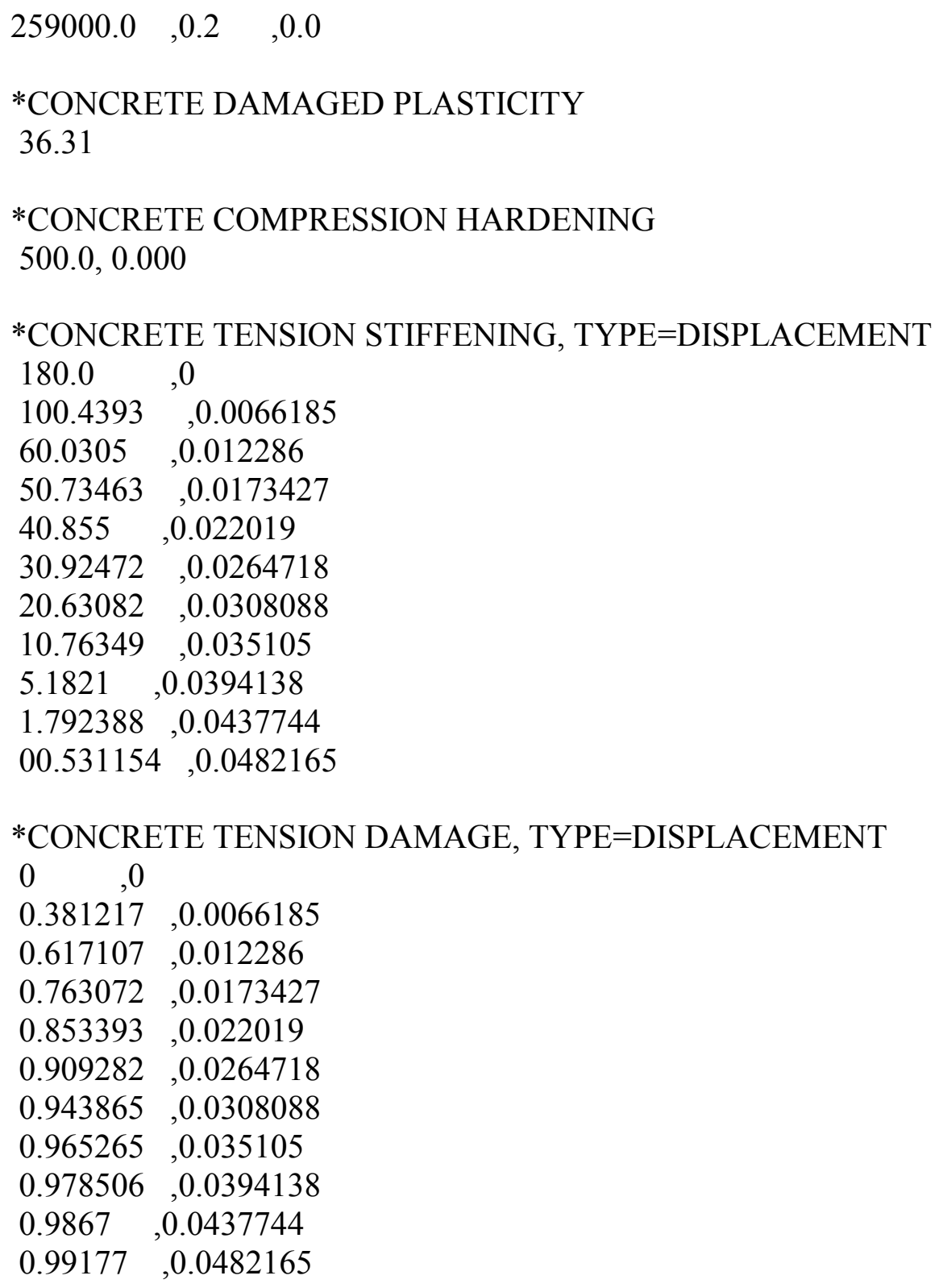

Cracking Model for Concrete

This model is designed for applications where the behavior is primarily dominated by tensile cracking. The compression response is assumed linear elastic. As with the previously discussed model the tensile response rises linearly until it reaches the failure stress after which a softening stress-strain response is observed. The post failure 
softening is defined by default via a stress vs cracking strain plot. An important distinction from the concrete damage plasticity model is that there is no permanent deformation on unloading from the softening region of the stress strain curve. As before a smeared crack approach is used to model the crack and individual crack is not tracked. The presence of crack is modeled as stiffness degradation of an element which is defined in the post failure material definition. The crack is assumed to form when the maximum principle stress in tension exceeds the failure stress of the material.

As for the previous model the tension stiffening is defined in terms of stress vs cracking strain data. Again this can lead to mesh sensitivity and hence it is recommended to provide the data in terms of either the fracture energy $G_{f}$ or in terms of stress - displacement data.

One advantage of this model is the ability to specify brittle failure for the model. At a particular material point of the model as the displacement or strain reaches a critical value the material point is considered to have failed and the stress in that element is set to zero. In certain cases such an element can undergo significant deformation and can result in a premature termination of the analysis, to overcome this issue the failed element can be removed from the mesh. This may result in a weaker response for the material as a deleted element is incapable of transmitting compressive stresses which is possible for an open crack.

The implementation of this model for the ceramic fracture models is shown below. Note that in our simulations we assumed a linear stress-strain softening response with 0.008 as the failure strain.

*MATERIAL, NAME=ceramic

*DENSITY

2.6000E-06, 0.0

*ELASTIC, TYPE = ISOTROPIC

$80000.0 \quad, 0.2 \quad, 0.0$

*BRITTLE CRACKING 
180.0,0.

$0 ., 0.008$

*BRITTLE SHEAR

$1 ., 0$.

$0 ., 0.008$

*BRITTLE FAILURE

0.008 


\section{APPENDIX C}

Here the Matlab code employed to obtain the initial random particle distribution is attached. The code is used to model the walnut-ceramic particle system.

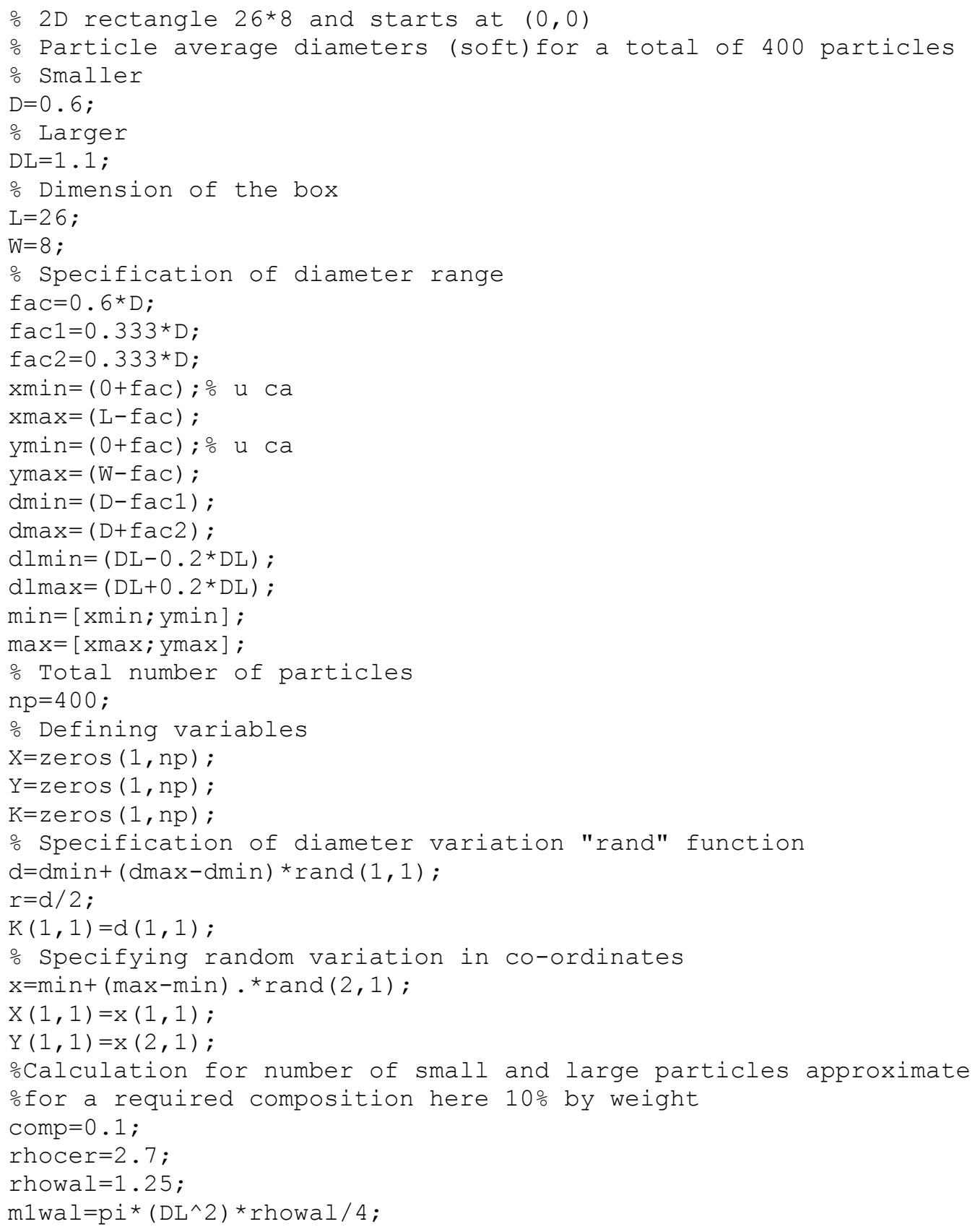




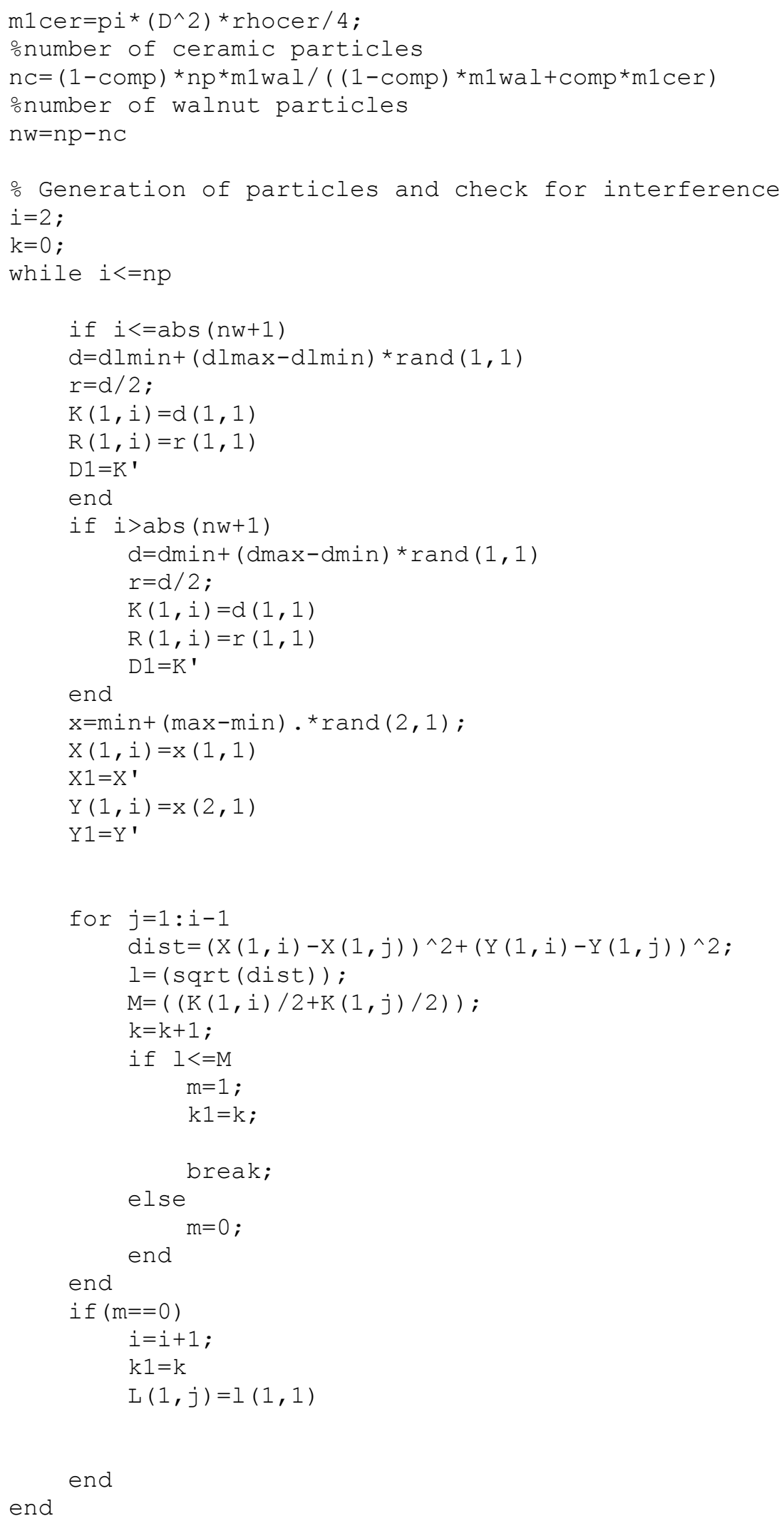




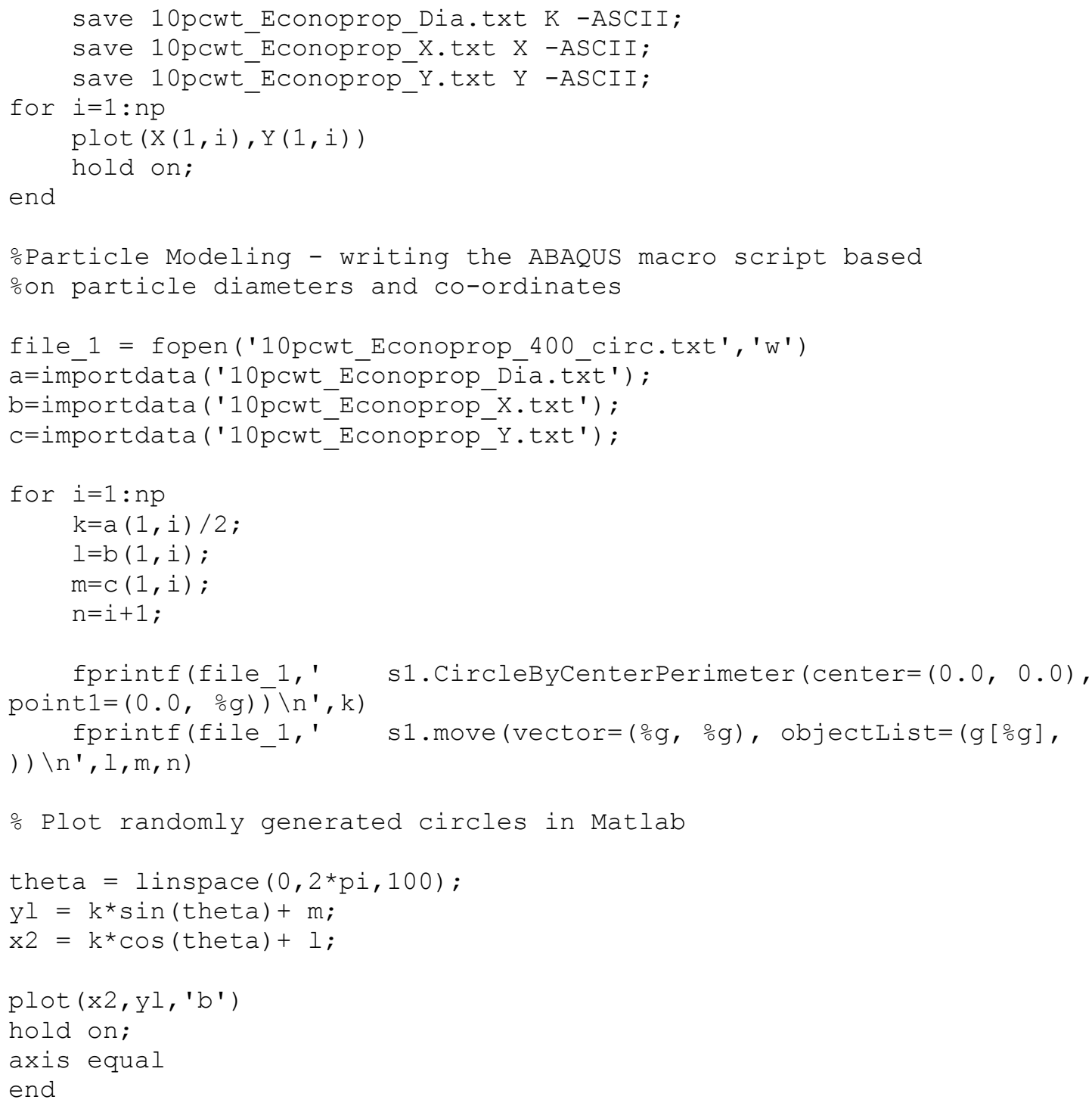

Note that, the above code was initially developed by Udaya Bhanu Sunku, of the Department of Aerospace Engineering, Texas A\&M University for a class project. The original code was modified with changes introduced to model polydispersity, modify particle spacing and introduce elliptical particles. 


\section{APPENDIX D}

Non-linear geometry condition is related to the change in the stiffness response of a model following its deformation in an analysis. This occurs when magnitudes of displacement are large enough to affect the structural response. The three main causes of Geometric nonlinearity as discussed in the ABAQUS/ Standard User's Manual [23] are:

- Large deflections or rotations.

- Snap through.

- Initial stresses or load stiffening.

An example of Geometric nonlinearity is that of a fishing rod undergoing large deflections, buckling of a column or the snap through of a large panel. In general as per ABAQUS/ Standar User's Manual [23] whenever the strains exceed a value of $\sim 5 \%$ non-linear geometry needs to be incorporated into the analysis. The Figure 90 below shows an example of effect of large deflection on a cantilever beam.
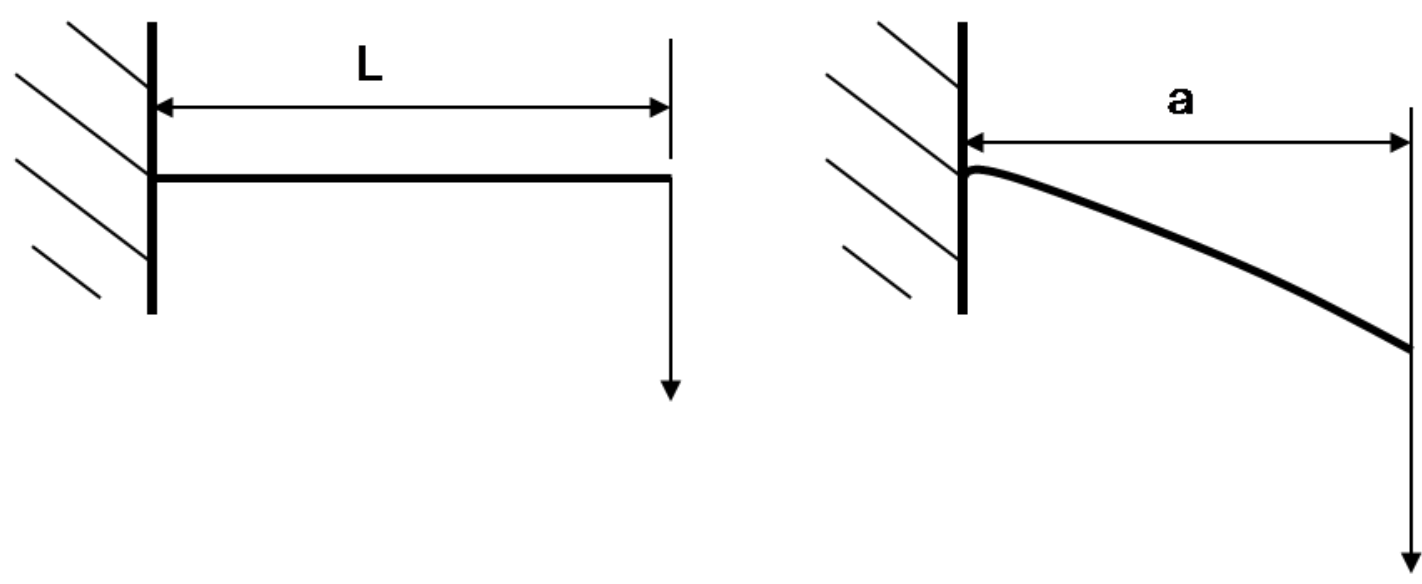

Fig. D. Large deflection in a cantilever beam 
In this case the tip of the cantilever beam undergoes a considerable axial deflection in addition to its transverse deflection. Thus the effective moment arm of the force is reduced $(\mathrm{a}<\mathrm{L})$ and hence the beam tends to give a stiffer response to additional deflection. This non-linear response because of change in model geometry under loading is an example of Geometric nonlinearity.

Geometric nonlinearity can be incorporated in an ABAQUS analysis by including the NLGEOM parameter with the *STEP option. This option takes into account the higher-order terms in the strain-displacement relations shown below. With this option incorporated the loading is incremental and the element stiffness matrix is updated at all the iterations. All the elements in ABAQUS have the ability to use a nonlinear formulation. The element output is true stress and logarithmic strain.

$$
\begin{aligned}
& \varepsilon_{X}=\frac{\partial u_{X}}{\partial X}+\frac{1}{2}\left[\left(\frac{\partial u_{X}}{\partial X}\right)^{2}+\left(\frac{\partial u_{Y}}{\partial Y}\right)^{2}+\left(\frac{\partial u_{Z}}{\partial Z}\right)^{2}\right] \\
& \varepsilon_{Y}=\frac{\partial u_{Y}}{\partial Y}+\frac{1}{2}\left[\left(\frac{\partial u_{X}}{\partial X}\right)^{2}+\left(\frac{\partial u_{Y}}{\partial Y}\right)^{2}+\left(\frac{\partial u_{Z}}{\partial Z}\right)^{2}\right] \\
& 2 \varepsilon_{X Y}=\frac{\partial u_{X}}{\partial X}+\frac{\partial u_{Y}}{\partial Y}+\left[\frac{\partial u_{X}}{\partial X} \frac{\partial u_{X}}{\partial Y}+\frac{\partial u_{Y}}{\partial X} \frac{\partial u_{Y}}{\partial Y}+\frac{\partial u_{Z}}{\partial X} \frac{\partial u_{Z}}{\partial Y}\right]
\end{aligned}
$$

Note that, the same discussion for geometric nonlinearity is presented in the Appendix of [59], which was part of my MS thesis. 


\section{APPENDIX E}

Material nonlinearity arises when the stress/ strain response follows a non-linear curve. In this case the stress response is dependent on strain and does not follow a linear relationship. Nonlinear material properties can be both elastic and plastic. As discussed in ABAQUS/ Standard User's Manual [23] the non-linear elastic properties include hyperelasticity, viscoelasticity, hypoelasticity etc. The inelastic properties include classical metal plasticity, rate dependent yield, anisotropic yield and creep, porous metal plasticity, cast iron plasticity, extended Drucker-Prager plasticity and creep, clay plasticity, crushable foam plasticity, concrete etc.

The classical metal plasticity is used to describe the yield and inelastic flow of metals at low temperatures where the creep effects are not important and loading is monotonic. The Mises or Hill yield surfaces associated with plastic flow are used in ABAQUS. Two definitions for work hardening are available, perfect plasticity and isotropic hardening both of these are described in Fig. E(a) and E(b).

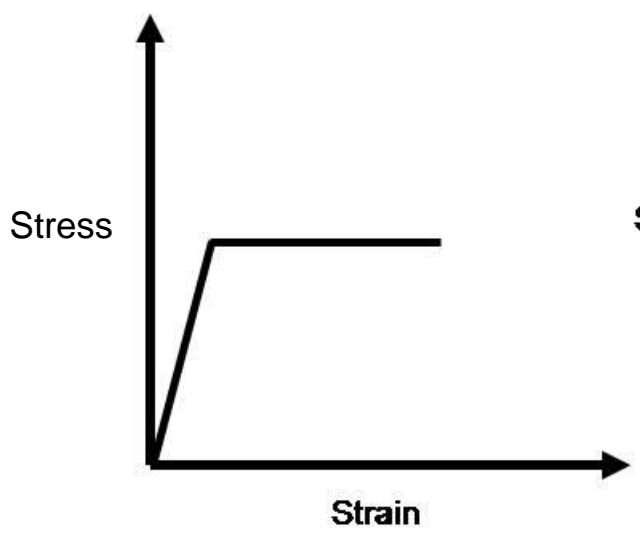

(a) Elastic Perfectly Plastic

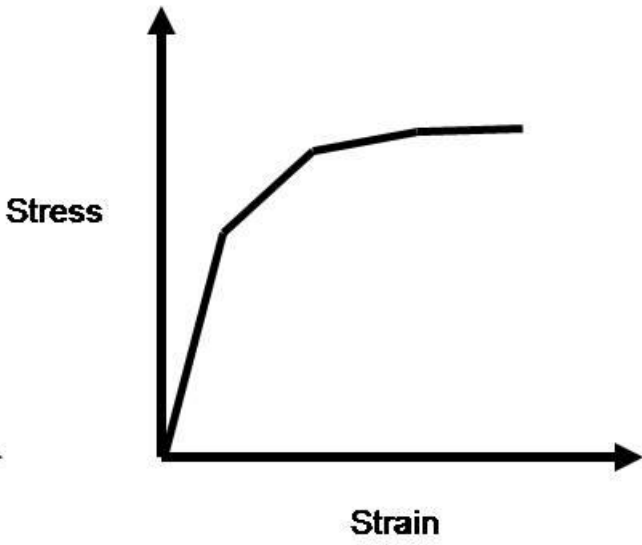

(b) Elastic Plastic Hardening

Fig. D. Stress strain relationship for (a) elastic perfectly plastic and (b) plastic with hardening 
Perfect plasticity implies that the yield stress is invariant with strain. With isotropic hardening the size of the yield surface changes uniformly in all directions, hence the yield stress increases or decreases in all directions uniformly as plastic straining takes place. Isotropic yielding is defined using the Mises yield surface. Classical metal plasticity can be incorporated in ABAQUS using the *PLASTIC card with the *MATERIAL option. The data for hardening behavior is incorporated as a true stress, and plastic strain data in a tabular format or by defining the yield stress in a user subroutine UHARD.

ABAQUS interpolates linearly between the input data points to obtain the material response and assumes that beyond the final data point the response is constant. Thus with an elastic perfectly plastic definition, stress in any element cannot exceed the yield stress and straining at a constant stress value takes place beyond this point. PEEQ (equivalent plastic strain) which is a scalar variable is used to represent a material's inelastic deformation. A PEEQ value greater than zero indicates material has yielded. PEEQ is defined by the following equation [23].

$\bar{\varepsilon}^{p l}=\left.\bar{\varepsilon}^{p l}\right|_{0}+\int_{0}^{t} \sqrt{\frac{2}{3} \dot{\varepsilon}^{p l}: \dot{\varepsilon}^{p l}} d t$

Here $\left.\bar{\varepsilon}^{p l}\right|_{0}$ is the initial equivalent plastic strain defined by the *INITIAL CONDITIONS option.

Note that, the same discussion for metal plasticity is presented in the Appendix of [59], which was part of my MS thesis. 


\section{VITA}

Name: $\quad$ Mandar Chaitanya Kulkarni

Address: Department of Mechanical Engineering, 3123 TAMU, College Station, TX 77843 , USA

Email Address: mandar.kulkarni@tamu.edu

Education: B.E, Mechanical Engineering, Sardar Patel University, India, 2004

M.S, Mechanical Engineering, Texas A\&M University, College Station, Texas, 2008

Ph.D., Mechanical Engineering, Texas A\&M University, College Station, Texas, 2009-2012 